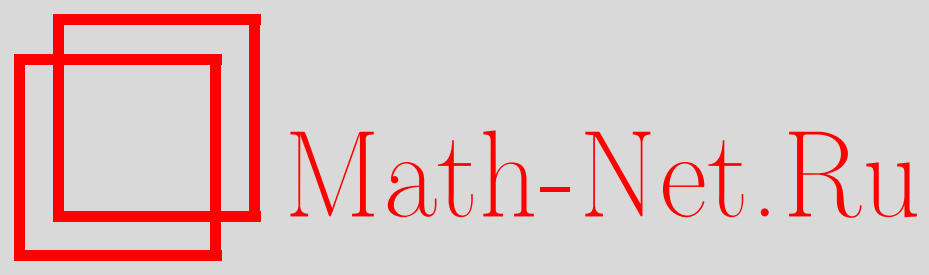

А. Г. Куликовский, А. П. Чугайнова, Классические и неклассические разрывы в решениях уравнений нелинейной теории упругости, УМH, 2008, том 63, выпуск 2, 85-152

DOI: https://doi.org/10.4213/rm9182

Использование Общероссийского математического портала Math-Net.Ru подразумевает, что вы прочитали и согласны с пользовательским соглашением http://www.mathnet.ru/rus/agreement

Параметры загрузки:

IP : 54.162 .85 .209

26 апреля 2023 г., $17: 32: 45$

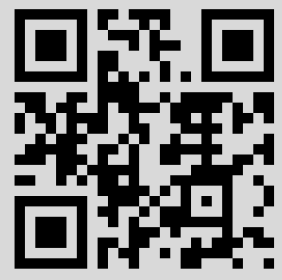


УДК 531.01

\title{
Классические и неклассические разрывы в решениях уравнений нелинейной теории упругости
}

\begin{abstract}
А. Г. Куликовский, А. П. Чугайнова
Предлагаемая работа посвящена изучению аналитическими и численными методами задач, связанных с распространением одномерных нелинейных волн малой амплитуды в упругих средах. Уравнения нелинейной теории упругости относятся к классу гиперболических систем, выражающих законы сохранения. Для возможности однозначного построения решений оказывается необходимым дополнить эти уравнения членами, позволяющими адекватно описывать реальные мелкомасштабные явления, в частности, структуру возникающих разрывов. Рассмотрено поведение нелинейных волн в двух случаях: когда мелкомасштабные процессы обусловлены вязкостью и когда помимо вязкости существенную роль играет дисперсия.
\end{abstract}

Библиография: 51 название.

\section{СОДЕРЖАНИЕ}

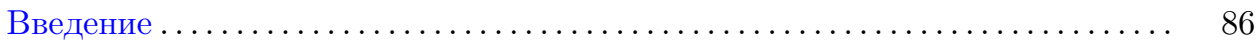

$\S 1$. Нелинейные волны малой амплитуды в упругих средах .......... 89

1.1. Волны Римана ................................ 91

1.2. Ударные волны . . . . . . . . . . . . . . . . . . . . . . . . . 93

1.3. Автомодельные задачи и неединственность решений ......... 96

$\S 2$. Нелинейные волны в вязко-упругих средах и проблемы устойчивости

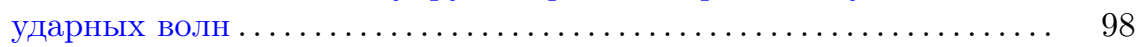

2.1. Структура квазипоперечных ударных волн.............. 98

2.2. Одномерные нестационарные решения. Описание решений в условиях неединственности автомодельных асимптотик . . . . . . . . 101

2.3. Взаимодействие нелинейных волн в слабоанизотропной среде.. . 104

2.4. Исследование устойчивости структуры ударных волн в вязкоупругой среде при взаимодействии с одномерными неоднород-

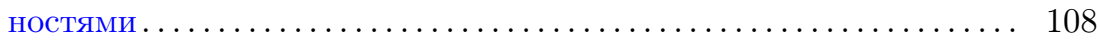

Работа выполнена в рамках и при финансовой поддержке научной программы Российской академии наук "Математические методы нелинейной динамики", а также при поддержке Российского фонда фундаментальных исследований (грант № 08-01-00618) и программы "Ведущие научные школы" (грант НШ1959.2008.1).

(C) А.Г. КуликовСКий, А. П. ЧугАйновА, 2008 
2.5. Устойчивость к двумерным возмущениям метастабильной ударной волны в вязко-упругой среде ................ 113

2.6. Выводы ..................................... 119

$\S 3$. Асимптотическое поведение нелинейных волн в упругих средах с дисперсией и диссипацией................................ 120

3.1. Структура разрывов ............................. 121

3.2. Неединственность решений автомодельной волновой задачи . . . 129

3.3. Решения полной системы уравнений в частных производных и их асимптотики............................ 130

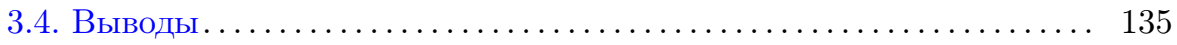

$\S 4$. Неклассические разрывы при распространении продольных волн в вязко-упругих стержнях со сложной нелинейностью ............

4.1. Разрывы со стационарной структурой. Задача о распаде произвольного разрыва. .............................. 137

4.2. Численное исследование задач с автомодельной асимптотикой.. 141

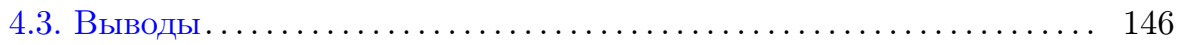

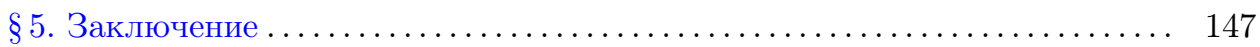

Список литературы ........................................... 148

\section{Введение}

Уравнения нелинейной теории упругости представляют собой гиперболическую систему, выражающую законы сохранения. Трудности в построении и изучении решений этой системы во многом связаны с необходимостью рассмотрения разрывных решений. На разрыве должна выполняться некоторая система соотношений, связывающих величины по обе стороны разрыва и его скорость. В число этих соотношений должны входить соотношения, обеспечивающие выполнение законов сохранения на разрыве. Будем называть разрыв классическим, если, во-первых, на разрыве не предполагается выполнения каких-либо других соотношений, кроме соотношений, выражающих законы сохранения, во-вторых, выполнены неравенства, обеспечивающие корректность системы граничных условий на разрыве (условия эволюционности, или условия Лакса), и условия неубывания энтропии. Если при выполнении этих условий имеет место положительное производство энтропии, то разрыв называется ударной волной. Если производство энтропии на разрыве равно нулю, то разрыв будем называть обратимым.

В простейших случаях, как, например, в газовой динамике совершенного газа, решения задач, которые строятся из непрерывных решений уравнений газовой динамики (гиперболических) и ударных волн, оказываются единственными. Однако известно также, что существуют гиперболические системы уравнений такие, что построение решений автомодельных задач с использованием непрерывных решений и ударных волн оказывается неоднозначным (см., например, [1], [2]). Для выделения единственных, физически обоснованных решений гиперболическая система уравнений во многих случаях дополнялась членами, которые пренебрежимо малы в областях, где изменение решений характеризуется некоторым пространственным масштабом $L$ рассматриваемых задач, а в узких областях, ширины много меньше $L$, оказывают существенное 
влияние на решение, делая его непрерывным. Такую систему уравнений будем называть расширенной или полной системой уравнений. Разрывам в решениях гиперболических систем уравнений соответствуют узкие переходные зоны в решениях полных систем уравнений. Решение полной системы уравнений внутри переходной зоны называется структурой разрыва. Требование существования структуры разрывов приводит к выделению разрывов, которые часто рассматриваются как реально существующие [3], т. е. допустимые для изучаемой системы гиперболических уравнений.

При неединственности решений в гиперболической постановке требование существования структуры у используемых разрывов в ряде случаев приводит к отбрасыванию некоторой части разрывов, в результате чего решение задачи может стать единственным, как это показано в [2], [4]. Может оказаться, что выделенное требованием существования структуры то или иное множество допустимых разрывов зависит от мелкомасштабных процессов в структуре, которые тем самым определяют решение задач в целом. Это было продемонстрировано на примерах, изложенных в [5], [6], а в [7] приведен пример гиперболической системы, для которой требование существования структуры разрывов, используемых при построении решения, не приводит к единственности решений.

В ряде случаев оказывается необходимым рассмотрение и введение в решения задач неклассических, особых разрывов, на которых кроме соотношений, следующих из законов сохранения, должны выполняться также некоторые дополнительные соотношения. К особым разрывам относятся хорошо известные фронты горения в газах [8], которые распространяются по горючей смеси так, что относительная скорость газа с обеих сторон от фронта меньше скорости звука. В качестве дополнительного условия на фронте обычно задается скорость фронта по отношению к газу перед фронтом. Эта скорость теоретически определяется как условие существования структуры фронта горения с учетом химической кинетики, теплопроводности и вязкости. Известны и другие задачи, в решениях которых существенную роль играют особые разрывы. В частности, исследованы разрывы, требование существования структуры которых приводит к нескольким дополнительным условиям, как, например, это имеет место, если газ, проходя через разрыв, приобретает или теряет электропроводность в присутствии магнитного поля [9], [10].

Во всех перечисленных случаях проводился выход за рамки гиперболической системы уравнений, причем добавляемые в эти уравнения члены существенно влияли на множество допустимых разрывов и их свойства, а следовательно, и на решения задач в целом. Поэтому, вообще говоря, оказывается недостаточным знание только вида гиперболических уравнений, а требуется знание полной системы уравнений, которая правильно описывает как крупномасштабные, так и мелкомасштабные явления.

Однако при рассмотрении явлений с точки зрения крупного масштаба часто бывает достаточно меньшего объема дополнительных сведений. Например, как уже упомянуто, в задачах с фронтами горения достаточно знания скорости этих фронтов. Будем называть гиперболической моделью гиперболическую систему уравнений для описания непрерывных решений и определение мно- 
жества допустимых разрывов, которые могут использоваться при построении решений.

Множество допустимых разрывов для одной и той же гиперболической системы уравнений может определяться различным образом, порождая разные гиперболические модели. В идеале множество допустимых разрывов совпадает с множеством разрывов, которые могут физически осуществляться. Однако последнее множество не всегда заранее известно. Уже упоминалось, что во многих случаях множество допустимых разрывов определяется как множество разрывов, которым соответствует решение задачи о структуре в рамках некоторой полной системы уравнений. При этом обычно дополнительно считается, что структура одномерна и стационарна, т. е. представляется бегущей волной. Такой подход к определению множества допустимых разрывов будет использоваться ниже. Однако при определенных условиях структура разрывов не представляется бегущей волной, а в ней происходят внутренние колебания. Внутренние колебания и неодномерность движения внутри структуры ранее были обнаружены в ряде задач механики сплошных сред и, в частности, в теории горения и детонации (см., например, изложение этого вопроса в работах [8], [11], [12]). Структура разрывов может быть стохастической, турбулентной, как это имеет место в гидравлическом прыжке [13]. Обнаружена колебательная структура разрывов и при распространении продольных волн по стержню (см. ниже §4). Поэтому выбор множества допустимых разрывов представляет во многих случаях сложную проблему.

Разумное задание множества допустимых разрывов не обязательно должно приводить к единственности решений, получаемых в рамках гиперболической модели. Это представляется очевидным, например, при рассмотрении задач о движении горючих смесей газов, когда крупномасштабное решение одной и той же задачи может содержать фронт детонации или фронт горения, а может вообще быть чисто газодинамическим. Кроме того, есть задачи, автомодельные решения которых не существуют в рамках разумно определяемого множества допустимых разрывов [14].

Всюду в этой статье предполагается, что в мелкомасштабных процессах, и в том числе в структуре разрывов, определенную роль играют диссипативные процессы, которые запрещают образование периодических незатухающих колебаний или близких к ним структур. В случае полного отсутствия диссипации возникают своеобразные явления, которые здесь не будут рассматриваться. С этими проблемами можно ознакомиться в книгах [15]-[18].

Излагаемые ниже результаты относятся к проблеме нелинейных волн малой амплитуды в упругих средах. Как уже упоминалось, уравнения нелинейной теории упругости [19], [20] представляют собой квазилинейную гиперболическую систему уравнений в частных производных, выражающую законы сохранения массы, импульса и энергии. Если в среде помимо упругих имеются также вязкие напряжения, то система приобретает свойства параболичности.

Свойства упругой среды определяются зависимостью ее внутренней энергии от деформации и энтропии. Ввиду предполагаемой малости деформаций внутренняя энергия представляется всюду в дальнейшем в виде многочлена по деформациям, причем в разложении по деформациям учитываются члены до четвертой степени включительно. Среда предполагается анизотропной, 
и свойства анизотропии в силу ее малости учитываются лишь в квадратичных членах. Анизотропия может быть изначально присущей среде, а может возникнуть в результате предварительной деформации среды, и ее наличие является случаем общего положения.

В изотропных упругих средах волны малых возмущений, рассматриваемые в линейном приближении, делятся на продольные и поперечные [19], [20]. В продольных волнах движение среды происходит по нормали к фронту волны, а в поперечных - в направлениях, параллельных фронту. Если нелинейность и анизотропия среды малы, то свойства волн меняются мало. В продольных волнах появляется малая поперечная составляющая движения, а в поперечных - малая продольная. Такие волны называются соответственно квазипродольными и квазипоперечными. Как было выяснено в [21] (см. также [22]), наиболее интересное поведение уже в случае малой нелинейности проявляют квазипоперечные волны. Ниже, в 11 и $\S 2$, дается обзор результатов исследования нелинейных квазипоперечных волн малой амплитуды в упругих и вязко-упругих слабоанизотропных средах, $\S 3$ посвящен изучению влияния дисперсии на эти волны. В $\S 4$ изучается распространение нелинейных продольных волн в стержнях, когда эффекты дисперсии проявляются при распространении не слишком длинных волн. В конце каждого параграфа и всей статьи кратко формулируются качественные выводы, полученные в результате исследования.

Наибольший интерес представляют задачи, для которых гиперболическая модель дает неединственное решение. Эти случаи подробно исследованы в предлагаемой работе с помощью численного построения решений полных систем уравнений с частными производными с выявлением гиперболических асимптотик.

Исследуется также устойчивость разрывов и соответствующих структур, существование которых может подвергаться сомнению из-за возможности их распада на систему волн.

В тех случаях, когда в мелкомасштабных явлениях помимо вязкости проявляются эффекты дисперсии, обнаружены новые свойства решений, которые, несомненно, должны иметь место и для других систем уравнений, мелкомасштабные явления в которых включают в себя диссипацию и дисперсию.

При изложении результатов в $\S 1-\S 3$ используется не система уравнений нелинейной теории упругости с дополнительными членами, описывающими мелкомасштабные явления, а приближенная упрощенная система, состоящая из двух гиперболических уравнений и описывающая две взаимодействующие между собой квазипоперечные волны, распространяющиеся в одну сторону с близкими скоростями. Эта система хорошо приближает решения исходной системы, если волны, связанные с другими семействами характеристик, малы по сравнению с изучаемыми. Вывод этой системы можно найти в [21], [22].

\section{§1. Нелинейные волны малой амплитуды в упругих средах}

Будем рассматривать движение упругой среды в форме плоских волн. Это означает, что решение зависит от единственной декартовой координаты и времени. Основные уравнения для непрерывных движений записываются в виде 
(см. монографию [20])

$$
\rho_{0} \frac{\partial v_{i}}{\partial t}=\frac{\partial}{\partial x}\left(\frac{\partial \Phi}{\partial u_{i}}\right), \quad \frac{\partial u_{i}}{\partial t}=\frac{\partial v_{i}}{\partial x}, \quad \frac{\partial S}{\partial t}=0, \quad i=1,2,3 .
$$

Здесь использован подход Лагранжа, в котором $x_{1}, x_{2}, x_{3} \equiv x$ - декартовы координаты начального состояния среды. Оси $x_{1}$ и $x_{2}$ лежат в плоскости волны, а ось $x_{3} \equiv x$ до деформации нормальна к ним, $\rho_{0}-$ плотность в начальном состоянии, $v_{i}=\partial w_{i} / \partial t-$ компоненты скорости, $u_{i}=\partial w_{i} / \partial x-$ производные по координате от компонент вектора перемещения $\mathbf{w}$. Функции $u_{i}, v_{i}$ и энтропия $S$ считаются функциями от $x$ и $t, \Phi=\rho_{0} U$, где $U$ - внутренняя энергия единицы массы, $\partial \Phi / \partial u_{i}$ представляют собой напряжения в плоскостях $x=$ const.

Вследствие геометрии задачи производные $\partial w_{\beta} / \partial x_{\alpha}(\alpha, \beta=1,2)$ не зависят от времени. Без ограничения общности производные $\partial w_{3} / \partial x_{\alpha}$ в плоских волнах можно считать равными нулю, так как эти величины могут быть обращены в нуль вращением твердого тела как единого целого. Таким образом, изменение деформации среды при ее движении в форме плоских волн может происходить только за счет изменения $u_{i}$. Функция $\Phi$ считается зависящей от $u_{i}, S$ и некоторых внутренних параметров, включающих параметры, которые определяют анизотропию [23]. В общем случае эти параметры могут зависеть от лагранжевых координат. В рассматриваемых ниже задачах о распространении волн малой амплитуды по однородному фону эти параметры, а также $\rho_{0}$ будут предполагаться постоянными. Система (1.1) полностью определяется заданием функции $\Phi\left(u_{i}, S\right)$, одной и той же для всех частиц среды.

Если среда изотропна в плоскости $\left(x_{1}, x_{2}\right)$ (случай волновой изотропии), то $u_{1}$ и $u_{2}$ входят в $\Phi$ в виде комбинации $u_{1}^{2}+u_{2}^{2}$. Таким образом, в случае волновой изотропии $\Phi$ зависит от $u_{1}^{2}+u_{2}^{2}, u_{3}$ и $S$. Будем рассматривать случаи, когда волновая анизотропия мала и $\Phi$ мало отличается от функции указанных аргументов, т. е.

$$
\Phi\left(u_{i}, S\right)=F\left(u_{1}^{2}+u_{2}^{2}, u_{3}, S\right)+q p\left(u_{1}, u_{2}, u_{3}, S\right)
$$

где $q$ - малый параметр анизотропии, $S$ - энтропия.

Уравнения (1.1) представляют собой гиперболическую систему в частных производных. Они выражают второй закон Ньютона, изменения деформаций под воздействием смещений и обратимость рассматриваемых процессов.

В изотропных упругих средах волны малых возмущений, рассматриваемые в линейном приближении, когда $F=\lambda\left(u_{1}^{2}+u_{2}^{2}\right)+\mu u_{3}^{2}, p \equiv 0(\lambda, \mu-$ коэффициенты Ламе), делятся на продольные и поперечные. В продольных волнах движение среды происходит по нормали к фронту волны (изменяется только $u_{3}$ ), а в поперечных - в направлениях, параллельных фронту (изменяются $u_{1}$ и $u_{2}$ ). Если нелинейность и анизотропия среды малы, то свойства волн меняются мало, причем в продольных волнах может появиться малая поперечная составляющая движения, а в поперечных - малая продольная. Такие волны называются соответственно квазипродольными и квазипоперечными. Квазипоперечным волнам соответствуют две близкие между собой характеристические скорости. Вследствие существенного различия характеристических скоростей квазипродольных и квазипоперечных волн волны этих семейств довольно 
быстро разойдутся, если начальные данные для этих волн заданы на конечном отрезке. Сложное совместное влияние малых нелинейности и анизотропии наиболее ярко проявляется в поведении квазипоперечных волн. В дальнейшем будем рассматривать волновой пакет ограниченной протяженности, составленный из квазипоперечных волн, соответствующих двум близким между собой характеристическим скоростям.

Слабонелинейные квазипоперечные волны, распространяющиеся по однородному фону в положительном направлении оси $x$, при малой анизотропии среды могут описываться упрощенной системой уравнений, которая следует из системы уравнений нелинейной теории упругости [21], [22], [24]

$$
\frac{\partial u_{\alpha}}{\partial t}+\frac{\partial}{\partial x}\left(\frac{\partial R\left(u_{1}, u_{2}\right)}{\partial u_{\alpha}}\right)=0, \quad \alpha=1,2,
$$

где

$$
\begin{gathered}
u_{\alpha}=\frac{\partial w_{\alpha}}{\partial x}=u_{\alpha}(x, t), \\
R\left(u_{1}, u_{2}\right)=\frac{1}{2} f\left(u_{1}^{2}+u_{2}^{2}\right)+\frac{1}{2} g\left(u_{2}^{2}-u_{1}^{2}\right)-\frac{1}{4} \varkappa\left(u_{1}^{2}+u_{2}^{2}\right)^{2}, \\
f, g, \varkappa=\text { const. }
\end{gathered}
$$

Здесь $g$ - малый параметр анизотропии, $\varkappa$ - постоянная с размерностью скорости, которая характеризует нелинейные эффекты, $f$ - характеристическая скорость при отсутствии нелинейности и анизотропии (т. е. при $\varkappa=0, g=0$ ). Знак упругой константы $\varkappa$ существенно влияет на поведение квазипоперечных простых и ударных волн. Знак $g$ считается совпадающим со знаком $\varkappa$, что определяет нумерацию переменных $u_{1}, u_{2}$. В системе уравнений (1.3) оставлены только главные члены, отвечающие за нелинейность и анизотропию.

Предположения, которые делаются при выводе уравнений (1.3), аналогичны тем, которые делаются при выводе известного уравнения Хопфа. Отличие заключается в одновременном рассмотрении возмущений, связанных с двумя близкими семействами характеристик.

Для рассматриваемых ниже малых значений $u_{\alpha}$ система (1.3) представляет собой гиперболическую систему с двумя семействами характеристик, которые соответствуют квазипоперечным волнам, распространяющимся в положительном направлении оси $x$.

Рассматривая систему уравнений (1.3) в системе координат, движущейся со скоростью $f$, и выбирая масштабы времени $T$ и длины $L$ так, что $L / T=g$, и масштаб измерения для $u_{\alpha}$ в виде $U=\sqrt{g / \varkappa}$, можно в (1.4) получить $f=0$, $g / \varkappa=1, \varkappa= \pm 1$. Таким образом, имеется только два качественно различных варианта записи системы уравнений (1.3). Если считать, что $u_{\alpha}$ имеют порядок $\varepsilon$ и эту величину выбрать в качестве единицы измерения $u_{\alpha}$, то новые $u_{\alpha}$ будут иметь порядок единицы, а новые $g$ и $\varkappa$ будут одного порядка величины, если в исходных уравнениях величина $g$ имела порядок $\varepsilon^{2} \varkappa$.

Далее будут рассматриваться автомодельные решения системы (1.3) вида $u_{\alpha}=u_{\alpha}(x / t)$. Эти решения состоят из волн Римана и ударных волн.

1.1. Волны Римана. Для гиперболической системы уравнений (1.3) найдем решение типа простой волны (волны Римана), т. е. такое, что

$$
u_{\alpha}=u_{\alpha}(\theta(x, t)) \text {, }
$$


где $\theta$ в общем случае - неизвестная функция. Эти решения изучены в работах [21], [25]. Подставив (1.5) в (1.3), получим систему обыкновенных дифференциальных уравнений для $u_{\alpha}$ :

$$
\begin{aligned}
& \left\{\begin{array}{l}
\left(R_{1,1}-c\right) \frac{d u_{1}}{d \theta}+R_{1,2} \frac{d u_{2}}{d \theta}=0, \\
R_{1,2} \frac{d u_{1}}{d \theta}+\left(R_{2,2}-c\right) \frac{d u_{2}}{d \theta}=0,
\end{array}\right. \\
& R_{\alpha, \beta}=\frac{\partial^{2} R}{\partial u_{\alpha} \partial u_{\beta}}, \quad \alpha, \beta=1,2 .
\end{aligned}
$$

Здесь

$$
c=-\frac{\partial \theta}{\partial t} / \frac{\partial \theta}{\partial x} .
$$

Система (1.6) имеет нетривиальное решение, если $c=c_{1,2}\left(u_{1}, u_{2}\right)$, где $c_{1,2}$ - характеристические скорости, являющиеся корнями определителя системы (1.6) $\left|R_{i, j}\left(u_{1}, u_{2}\right)-c \delta_{i j}\right|$. Из (1.7) следует, что $\theta$ и, соответственно, $u_{\alpha}(\theta)$ постоянны на каждой из характеристик этого уравнения, представленных прямыми линиями в плоскости $(x, t)$ и определяемых формулой

$$
\frac{d x}{d t}=c_{\alpha}\left(u_{1}, u_{2}\right), \quad \alpha=1,2 .
$$

На плоскости $\left(u_{1}, u_{2}\right)$ равенство $c_{1}=c_{2}$ выполняется в точках $u_{1}=0$, $u_{2}= \pm \sqrt{G}, G=g / \varkappa$ (предполагается, что $\left.g / \varkappa>0\right)$. Интегральные кривые волн Римана касаются собственных векторов матрицы $R_{\alpha, \beta}$ и на плоскости $\left(u_{1}, u_{2}\right)$ образуют два ортогональных семейства кривых (рис. 1.1) [21], [22], [25]. Оси $u_{1}$ и $u_{2}$ для множества интегральных кривых являются осями симметрии. Решения, соответствующие $c_{1}$ и $c_{2}\left(c_{1}<c_{2}\right)$, называются медленными и быстрыми квазипоперечными простыми волнами соответственно.

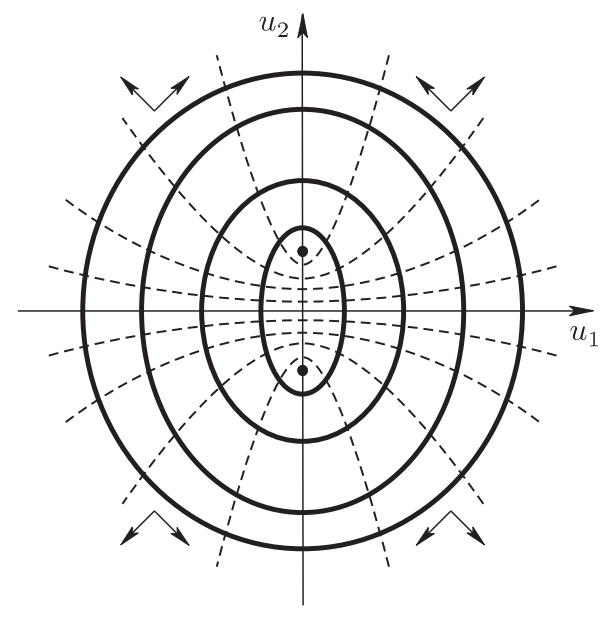

Рис. 1.1. Интегральные кривые простых волн $(\varkappa>0)$

Рассмотрим уравнения (1.6) для $c=c_{1}$ и $c=c_{2}$. Точки, в которых $c_{1}=c_{2}$, являются особыми точками обоих семейств интегральных кривых. В случае 
$\varkappa>0$ эллипсоподобные (сплошные, рис. 1.1) кривые соответствуют быстрым волнам $c=c_{2}$, а кривые гиперболического вида (пунктирные) отвечают медленным волнам $c=c_{1}$. Для $\varkappa<0$ ситуация является противоположной. В принятой аппроксимации полная картина интегральных кривых квазипоперечных волн всегда одинакова с точностью до масштаба, определяемого $\sqrt{G}$, и не зависит от знака $\varkappa$. При $G \rightarrow 0$ (или $u_{1}^{2}+u_{2}^{2} \rightarrow \infty$ ) интегральные кривые трансформируются в окружности и лучи, что отвечает волнам изотропного случая [20]. На рис. 1.1 стрелки указывают направление убывания $c_{\alpha}$ вдоль интегральных кривых для $\varkappa>0$. Если $\varkappa<0$, то $c_{\alpha}$ убывают в противоположных направлениях. В дальнейшем будут рассматриваться автомодельные волны Римана, когда $\theta=x / t$. Изменение величин в таких волнах соответствует отрезку интегральной кривой, вдоль которой $c$ убывает при убывании $\theta=x / t$. Это обеспечивает расширение волны со временем, так что ее передний фронт движется быстрее, чем задний.

1.2. Ударные волны. Системе уравнений (1.3) соответствуют соотношения на разрыве [21], [26], которые выражают законы сохранения поперечных компонент импульса и должны выполняться при отсутствии внешних воздействий, сосредоточенных на поверхности разрыва:

$$
\left[\frac{\partial R}{\partial u_{\alpha}}\right]-W\left[u_{\alpha}\right]=0, \quad \alpha=1,2 .
$$

Здесь $W=d x / d t$ - лагранжева скорость разрыва. Квадратными скобками обозначены скачки соответствующих величин на фронте разрыва. Исключив $W$ из системы уравнений (1.8), получим уравнение ударной адиабаты:

$$
\left(u_{1}^{2}+u_{2}^{2}-U_{1}^{2}-U_{2}^{2}\right)\left(U_{1} u_{2}-U_{2} u_{1}\right)+\frac{2 g}{\varkappa}\left(u_{1}-U_{1}\right)\left(u_{2}-U_{2}\right)=0 .
$$

Здесь $U_{1}$ и $U_{2}$ обозначают значения величин $u_{1}$ и $u_{2}$ перед разрывом. Величины за разрывом удовлетворяют уравнению ударной адиабаты и обозначаются по-прежнему $u_{1}$ и $u_{2}$. На рис. $1.2 a, c$ и рис. 1.3 a на плоскости $\left(u_{1}, u_{2}\right)$ изображены ударные адиабаты (точка $A\left(U_{1}, U_{2}\right)$ отвечает состоянию перед разрывом) для значений параметров $U_{1}=2, U_{2}=0.4, \varkappa=4$ (рис. $\left.1.2 a\right), U_{1}=1$, $U_{2}=0.1, \varkappa=2$ (рис. $\left.1.2 c\right)$ и $U_{1}=1, U_{2}=0.1, \varkappa=-2$ (рис. $1.3 a$ ). В каждом из представленных вариантов $g=1$.

Не все состояния $u_{1}, u_{2}$, принадлежащие ударной адиабате, могут быть состояниями за фронтом скачка. Разрывные решения, кроме того, должны удовлетворять условиям эволюиионности [27], выражающим необходимые условия корректности граничных условий на фронте по отношению к малым одномерным возмущениям. Условия эволюционности состоят в том, что число различных типов малых возмущений, уходящих от разрыва в обе стороны (число уходящих характеристик), должно быть на единицу меньше числа имеющихся на разрыве условий. Условия эволюционности, полученные в предположении, что на разрыве выполнены только законы сохранения и нет дополнительных соотношений другого происхождения, будут называться априорными условиями эволюиионности. Эти условия называются также условиями Лакса [28]. Если соотношения на разрывах представлены системой (1.8), то априорные условия 


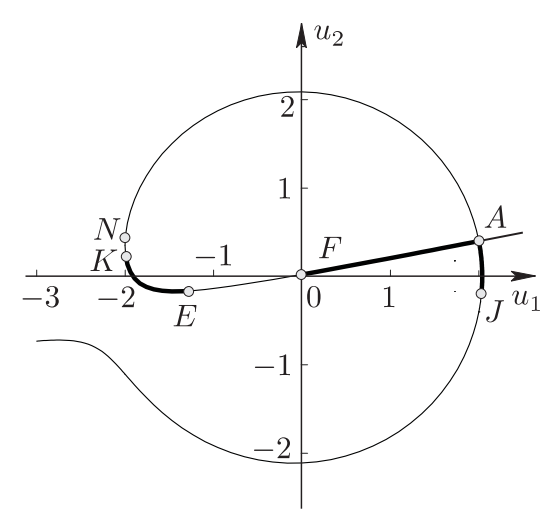

a

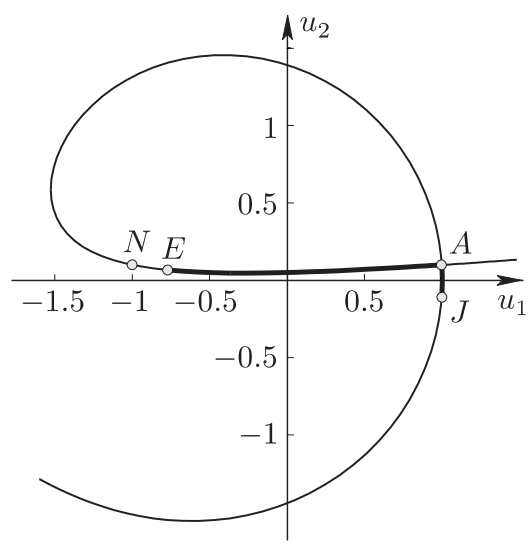

$c$

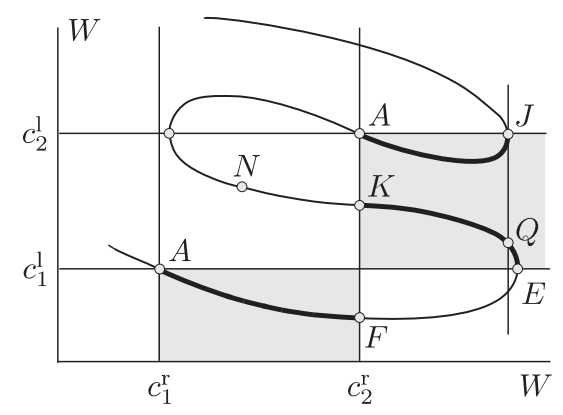

$b$

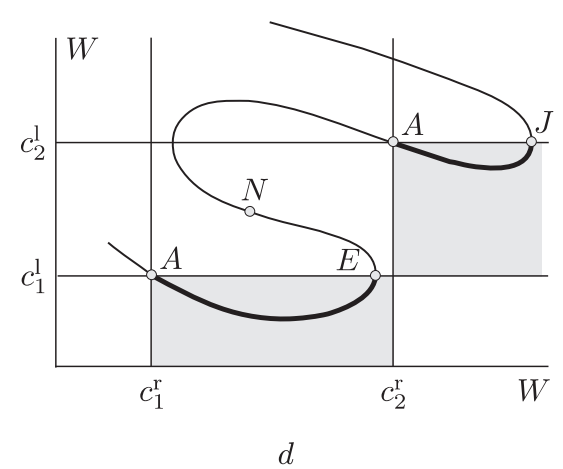

Рис. 1.2. Ударные адиабаты и диаграммы эволюционности для различных значений параметров $g, \varkappa, U_{\alpha}(\varkappa>0)$

эволюционности представлены двумя системами неравенств [21], [26]:

$$
\begin{aligned}
& c_{2}^{\mathrm{r}} \leqslant W, \quad c_{1}^{\mathrm{l}} \leqslant W \leqslant c_{2}^{\mathrm{l}}, \\
& c_{1}^{\mathrm{r}} \leqslant W \leqslant c_{2}^{\mathrm{r}}, \quad W \leqslant c_{1}^{\mathrm{l}} .
\end{aligned}
$$

Здесь и далее верхние индексы 1 и r будут характеризовать значение величин за и перед разрывом.

Соотношения (1.10) и (1.11) определяют эволюционные разрывы, которые называются быстрыми и медленными ударными волнами. Для наглядного представления неравенств (1.10) и (1.11) удобно пользоваться диаграммой эволюционности (рис.1.2 b, $d$, рис. $1.3 b$ ), где по взаимно ортогональным осям отложены скорости, участвующие в неравенствах, и тем самым выделены на плоскости области, где выполняется каждое из неравенств. При фиксированном состоянии перед фронтом скорость разрыва, а также скорости характеристик воспроизводятся на горизонтальной оси диаграммы эволюционности в одном 


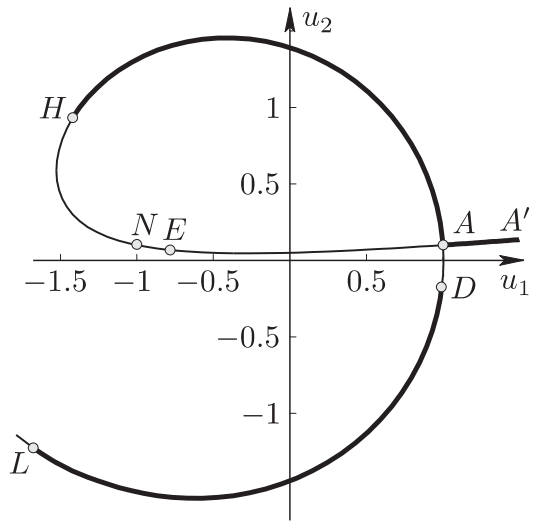

a

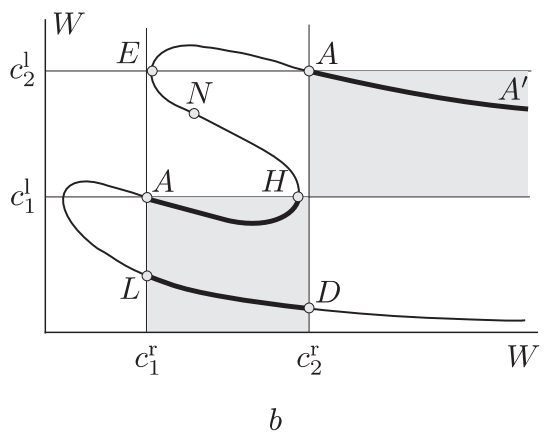

b

Рис. 1.3. Ударная адиабата и диаграмма эволюционности для сред с $\varkappa<0$

и том же масштабе. Вдоль вертикальной оси сохранены только неравенства между $W, c_{1}^{\mathrm{l}}$ и $c_{2}^{\mathrm{l}}$. Области, где выполняются оба неравенства каждой из систем $(1.10),(1.11)$, образуют на плоскости затененные прямоугольники (рис.1.2 $b, d$, $1.3 \mathrm{~b})$.

Соотношения на разрыве (1.8) позволяют найти скорость $W$ и другие величины вдоль ударной адиабаты и отобразить ударную адиабату на указанную плоскость. При этом значения $W$ правильно представляются по горизонтальной оси и лишь качественно по вертикальной. На рис. $1.2 b, d, 1.3 b$ начальная точка $A$ представлена двумя точками, в силу ее положения на двух пересекающихся ветвях ударной адиабаты. Отрезки ударной адиабаты, которые попали в эволюционные прямоугольники, соответствуют эволюционным быстрым ударным волнам в верхнем эволюционном прямоугольнике и медленным в нижнем. Соответственно такие же отрезки на ударной адиабате на плоскости $\left(u_{1}, u_{2}\right)$ на рис.1.2 $a, c, 1.3$ a показаны жирными сплошными отрезками, концы которых обозначены теми же буквами, что и на диаграммах эволюционности.

В случае $\varkappa>0$ ситуация, изображенная на рис. $1.2 a, b$, соответствует состояниям перед разрывом с относительно большими $U_{1}$ и $U_{2}$ (такими, например, что $\left.\varkappa\left(U_{1}^{2}+U_{2}^{2}\right)>2 g\right)$. В этом случае имеется три эволюционных отрезка: отрезки $A J$ и $K E$ отвечают возможным состояниям за эволюционными быстрыми ударными волнами, отрезок $A F$ - возможным состояниям за медленными ударными волнами. При достаточно малых $U_{1}$ и $U_{2}$ ударная адиабата и диаграмма эволюционности имеют вид, представленный на рис. $1.2 c, d$ соответственно. В этом случае имеется два эволюционных отрезка. В [21] на плоскости $\left(U_{1}, U_{2}\right)$ исследована граница, разделяющая случаи $1.2 a, b$ и $1.2 c, d$.

На рис. $1.2,1.3$ отмеченные точки $F, E, K, J, D, L, H$ называются точками Жуге, в этих точках скорость ударной волны совпадает с одной из характеристических скоростей перед или за скачком. Точки Жуге являются граничными точками эволюционных интервалов. Ударные волны, отвечающие точкам Жуге, играют важную роль в построении автомодельных решений. $\mathrm{K}$ ним в автомодельных решениях соответственно спереди или сзади могут непосред- 
ственно примыкать волны Римана того же типа, что и ударные волны (быстрая к быстрой, медленная к медленной).

На всех рисунках $1.2,1.3$ отмечена характерная точка $N$, которая на плоскости $\left(u_{1}, u_{2}\right)$ симметрична точке $A$ относительно оси $u_{2}$. В соответствующем скачке $A \rightarrow N$, не удовлетворяющем неравенствам (1.10), (1.11), величина $u_{1}$ меняет знак, а величина $u_{2}$ неизменна.

Упрощенная система уравнений (1.3) не включает энтропию или какую-либо другую термодинамическую переменную, поэтому условие неубывания энтропии на разрыве должно выражаться как условие невозрастания механической энергии, которое приводится к виду (см. [21])

$$
\frac{1}{2}\left(\left(\frac{\partial R}{\partial u_{\alpha}}\right)^{1}+\left(\frac{\partial R}{\partial u_{\alpha}}\right)^{\mathrm{r}}\right)\left[u_{\alpha}\right] \leqslant 0 .
$$

В рассматриваемом случае все эволюционные разрывы удовлетворяют требованию неубывания энтропии [21].

1.3. Автомодельные задачи и неединственность решений. Автомодельная задача о распаде произвольного начального разрыва в нелинейно упругой среде состоит в том, что в начальный момент времени $t=0$ имеются два однородных состояния: $u_{\alpha}=U_{\alpha}$ справа (при $\left.x>0\right)$ и $u_{\alpha}=u_{\alpha}^{*}$ слева (при $x<0$ ) от плоскости $x=0$, которая служит фронтом начального разрыва $(\alpha=1,2)$. Задача состоит в нахождении последовательности волн, распространяющихся в каждое полупространство от границы начального разрыва. Во многих случаях достаточно рассмотреть решение по одну сторону от плоскости $x=0$, например, для $x \geqslant 0$. Такая постановка в газовой динамике называется задачей о поршне. Задача о поршне для модели, задаваемой равенствами (1.3), (1.8), очевидно совпадает с задачей о распаде произвольного разрыва. Формулировка задачи о поршне такова: $u_{\alpha}=U_{\alpha}$ для $t=0, x \geqslant 0$ и $u_{\alpha}=u_{\alpha}^{*}$ для $t \geqslant 0$, $x=0$. Решение этой задачи является также приближенным решением задачи о поршне в исходной формулировке, соответствующей уравнениям (1.1).

В работах [29], [30] построено решение сформулированной автомодельной задачи. Решение состоит из последовательности автомодельных волн Римана и ударных волн, следующих в порядке убывания скорости. Построенное решение принципиально различается для сред с $\varkappa>0$ и $\varkappa<0$ и зависит от параметра анизотропии $g$. В [29] отмечено, что у автомодельной задачи о внезапном изменении напряжений на границе упругого полупространства (задачи о поршне) в некоторой области параметров, характеризующих задачу (области неединственности), могут существовать два решения. Геометрия области неединственности зависит от параметров $U_{\alpha}, g, \varkappa$.

На рис. 1.4 заштрихована область неединственности для среды с $\varkappa>0$ при достаточно больших значениях величин $U_{\alpha}$ (таких, что $\varkappa\left(U_{1}^{2}+U_{2}^{2}\right)>2 g$ ). Точки отрезков $A J$ и $K E$ представляют собой состояния за эволюционными быстрыми ударными волнами, распространяющимися по состоянию $A$, точки отрезка $A F$ - за медленными. Область неединственности решения (область значений $u_{\alpha}^{*}$, для которых имеются два решения) ограничена отрезком $P E$ ударной адиабаты из начальной точки $A\left(U_{1}, U_{2}\right)$, эволюционным отрезком $Q P$ ударной адиабаты, построенной из точки $Q$ как начальной точки, отрезками $Q Q^{\prime}$ 
и $E E^{\prime}$ интегральных кривых простых неопрокидывающихся волн. Точка $Q$ определена условием, что скорость ударной волны $A \rightarrow Q$ равна скорости ударной волны $A \rightarrow J$ (рис.1.2 b). На рис. 1.4 пунктирными линиями обозначены используемые в решении участки интегральных кривых простых волн. Линии, состоящие из точек, представляют состояния за ударными волнами Жуге, которые используются при построении решений.

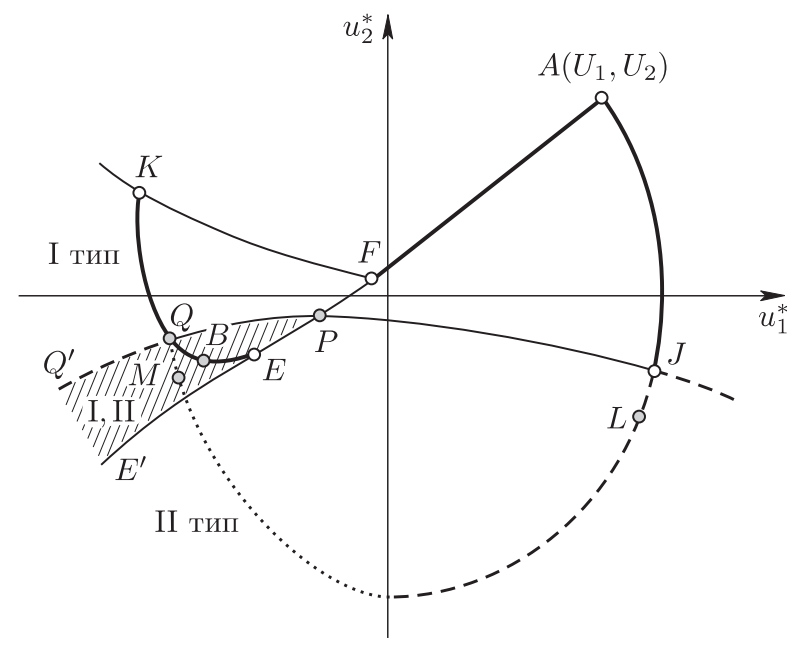

Рис. 1.4. Решение задачи о поршне для $\varkappa>0$ : зависимость решения от граничных данных $\left.u_{\alpha}\right|_{x=0}=u_{\alpha}^{*}$ при $\left.u_{\alpha}\right|_{t=0}=U_{\alpha}$. Заштрихована область двузначности решения.

Для рассматриваемых сред с $\varkappa>0$ одно из решений в области неединственности (решение первого типа) состоит из быстрой ударной волны из точки $A$ в точку эволюционного отрезка $Q E$ (в последующем изложении эта волна будет называться ударной волной типа $Q E)$ и следующей за ней медленной ударной или простой волны. Второе решение (решение второго типа) при $\varkappa>0$ содержит "сложную" быструю волну, состоящую из быстрой ударной волны Жуге $A \rightarrow J$ (точка $J$ - точка Жуге по состоянию за скачком), быстрой простой волны $J \rightarrow L$ и быстрой ударной волны Жуге $L \rightarrow M$ (эта волна того же типа, что и $A \rightarrow K$, условие Жуге выполнено в точке $L$ ), см. рис. 1.4. За "сложной" быстрой волной в решении второго типа с меньшей скоростью следует медленная волна - ударная или простая. Каждое из двух решений непрерывно продолжается в одну из соседних областей (рис. 1.4). Оба решения совпадают на кривой $Q^{\prime} Q P$, но различны на кривой $P E E^{\prime}$.

Для сред с $\varkappa<0$ тоже имеется область, где одновременно могут осуществляться два различных решения [21]. В отличие от случая $\varkappa>0$ область неединственности при $\varkappa<0$ существует при любых соотношениях между начальными деформациями и параметром анизотропии.

Неединственность решения была обнаружена при фактическом построении решения задачи [21], [29]. Однако можно предвидеть ее появление и дать до- 
статочный признак неединственности, не получая полного решения, а воспользовавшись видом диаграммы эволюционности [31].

Согласно изложенному, решения автомодельных задач нелинейной теории упругости могут быть неединственными. Неединственность обнаружена для возмущений малой амплитуды в упругой среде с уравнением состояния общего типа. Неединственность присуща как полной системе уравнений (1.1), так и упрощенной системе уравнений (1.3) при $g \neq 0$. Неединственность исчезает в вырожденном случае отсутствия волновой анизотропии $(g=0)$, так как при $g \rightarrow 0$ решения стремятся к совпадению [32].

Выше предполагалось, что только априорно эволюционные разрывы могут быть использованы для построения решений. Это оказалось справедливым, если упругая среда рассматривается как предел вязко-упругой среды, а допустимыми считаются разрывы со стационарными структурами (§2). При других сглаживающих разрывы механизмах множество допустимых разрывов становится другим (§3) и, соответственно, возникают другие решения автомодельных задач.

\section{§ 2. Нелинейные волны в вязко-упругих средах и проблемы устойчивости ударных волн}

Неединственность решений автомодельных задач представляет собой серьезный дефект уравнений нелинейной теории упругости, не позволяющий однозначно определять решение уравнений даже в случае гладких начальных условий. Можно попытаться выделить единственное решение, сузив множество допустимых разрывов. Одним из наиболее строгих правил отбора реализующихся участков ударной адиабаты служит исследование структуры ударных волн. Например, в [2], [4] подобное исследование для продольных волн в случае сложной ударной адиабаты позволило выделить единственное решение. Постановка этой задачи требует уточнения модели среды и тем самым выходит за рамки классической теории упругости.

В п. 2.1 будут рассмотрены структуры для всех квазипоперечных ударных волн с учетом вязких напряжений. При этом особое внимание будет уделяться быстрым ударным волнам, которые соответствуют скачкам из начального состояния $A$ в состояния на отрезке $Q E$ ударной адиабаты для сред с $\varkappa>0$ (рис. 1.2), и медленным ударным волнам - скачкам из точки $A$ в состояния на отрезке $L D$ для сред с $\varkappa<0$ (рис. 1.3). Если бы эти эволюционные ударные волны по какой-то причине потеряли право на существование, то решение в заштрихованной на рис.1.4 области оказалось бы единственным и в то же время это не отразилось бы на решениях, построенных для всех остальных областей плоскости $\left(u_{1}^{*}, u_{2}^{*}\right)$.

2.1. Структура квазипоперечных ударных волн. Рассмотрим стационарные структуры ударных волн. В качестве диссипативного механизма в динамические уравнения теории упругости (1.3) добавим члены, учитывающие дополнительные напряжения, вызванные вязкостью. Это самый простой из диссипативных механизмов, который используется в теории деформируемого твердого тела. Он положен в основу модели вязко-упругой среды Кельвина- 
Фойхта и обеспечивает непрерывность решений. Систему уравнений (1.3) для квазипоперечных волн малой амплитуды, распространяющихся в положительном направлении оси $x$, в этой модели можно записать в следующем виде (см. [21]):

$$
\frac{\partial u_{\alpha}}{\partial t}+\frac{\partial}{\partial x}\left(\frac{\partial R\left(u_{1}, u_{2}\right)}{\partial u_{\alpha}}\right)=\mu \frac{\partial^{2} u_{\alpha}}{\partial x^{2}}, \quad \alpha=1,2 .
$$

Уравнения (2.1) записаны в том же приближении, что и (1.3), и отличаются от них только наличием правой части, где $\mu$ - кинематический коэффициент вязкости, считающийся постоянным. Путем перехода в движущуюся систему координат и подходящего изменения масштабов длины $(L)$ и времени $(T)$ наряду с получением равенств $f=0, g=1,|\varkappa|=1$ (когда $T$ и $L$ были связаны только соотношением $L / T=g$ ) можно также обратить $\mu$ в единицу, умножая $L$ и $T$ на один и тот же множитель. Это обстоятельство используется для обнаружения подобия решений задач. В частности, если начальные условия заданы при $t=0$, то решение задачи Коши при $t=T, \mu=\mu_{0}, f=0$ отличается только масштабом по оси $x$ от решения другой задачи Коши при $t=1, \mu=\mu_{0} / T$, $f=0$ и с начальными условиями, "сжатыми" по оси $x$ в $T$ раз. Таким образом, рассмотрение поведения нестационарных решений задач при $t \rightarrow \infty, \mu=\mathrm{const}$ позволяет судить о поведении решений при $t=$ const, $\mu \rightarrow 0$.

Исследуем стационарную структуру квазипоперечных разрывов. Для системы уравнений (2.1) разыскиваются решения вида $u_{\alpha}=u_{\alpha}(\xi), \xi=-x+W t$, $\alpha=1,2$, где $W=$ const - скорость разрыва. При $\xi \rightarrow-\infty$ величины $u_{\alpha}$ стремятся к значениям $U_{\alpha}$, характеризующим состояние перед разрывом, а при $\xi \rightarrow+\infty$ величины $u_{\alpha}$ принимают значения, соответствующие состоянию за разрывом, т. е. состоянию, принадлежащему некоторой точке ударной адиабаты. При $\mu \rightarrow 0$ эти решения переходят в разрывы, изменение величин в которых совпадает с изменением величин в соответствующем квазипоперечном разрыве. Функции $u_{\alpha}(\xi)$ должны удовлетворять уравнениям

$$
\frac{\partial}{\partial \xi}\left(\frac{\partial}{\partial u_{\alpha}}\left(-R\left(u_{1}, u_{2}\right)+\frac{1}{2} W u_{\alpha}^{2}\right)\right)=\mu \frac{\partial^{2} u_{\alpha}}{\partial \xi^{2}}, \quad \alpha=1,2 .
$$

Интегрируя, будем иметь

$$
\begin{gathered}
\mu \frac{\partial u_{\alpha}}{\partial \xi}=\frac{\partial Z}{\partial u_{\alpha}}, \\
Z\left(u_{1}, u_{2}\right)=-R\left(u_{1}, u_{2}\right)+\frac{1}{2} W\left(u_{1}^{2}+u_{2}^{2}\right)+Q_{1} u_{1}+Q_{2} u_{2}, \\
Q_{\alpha}=U_{\alpha}\left(f-W+(-1)^{\alpha} g-\varkappa\left(U_{1}^{2}+U_{2}^{2}\right)\right) .
\end{gathered}
$$

Постоянные интегрирования $Q_{\alpha}$ определены из условий при $\xi \rightarrow-\infty$, т. е. перед скачком: $u_{\alpha}=U_{\alpha},\left(d u_{\alpha} / d \xi\right)_{-\infty}=0$.

Чтобы задача о структуре имела решение, у системы (2.2) должны существовать интегральные кривые, соединяющие на плоскости $\left(u_{1}, u_{2}\right)$ начальную точку $A\left(U_{1}, U_{2}\right)$ с точкой $\left(u_{1}, u_{2}\right)$, изображающей состояние за разрывом. Эти состояния для системы (2.2) должны быть стационарными (особыми) точками. 

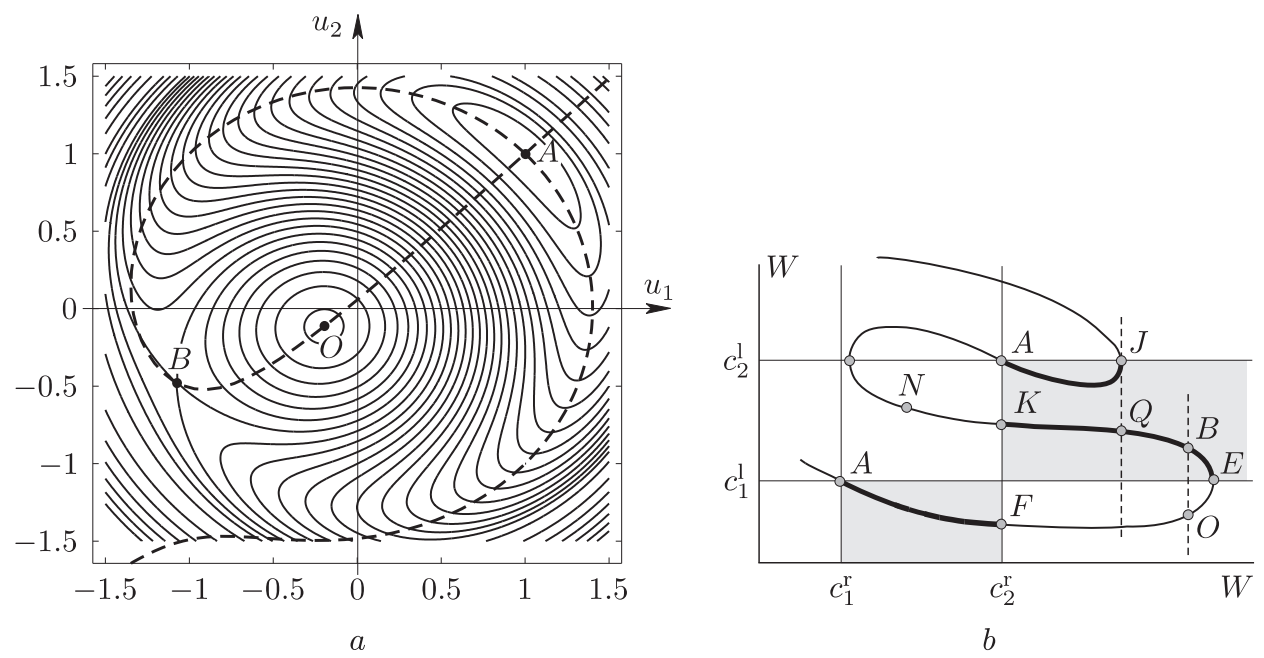

Рис. 2.1. Структура квазипоперечных ударных волн в средах с $\varkappa>0$

Были рассмотрены все значения $W$, которые соответствуют эволюционным ударным волнам в средах с $\varkappa>0$ [33] и с $\varkappa<0$ [34]. Для каждого $W$ определены типы особых точек, построены интегральные кривые и указаны направления на них, соответствующие росту $\xi$ (т. е. росту $t$ при $x=$ const). Особое внимание привлекают структуры наиболее важных для проблемы неединственности ударных волн, у которых состояние за фронтом принадлежит эволюционному участку $Q E$ ударной адиабаты (для сред с $\varkappa>0$ ) или участку $D L$ (для $\varkappa<0)$.

На рис. 2.1 а представлена картина линий уровня функции $Z\left(u_{1}, u_{2}\right)$ для значений параметров $U_{1}=U_{2}=1, f=1, g=0.03, \varkappa=0.5, W=0.13$, пунктирной линией на этом графике изображена ударная адиабата для выбранных параметров. На рис. 2.1 b приведена диаграмма эволюционности, на которой выбранное значение $W$, соответствующее скорости ударной волны типа $Q E$, изображено прямой $B O$. Для системы (2.2) интегральные кривые ортогональны линиям уровня функции $Z$. Для быстрой ударной волны $A \rightarrow B$ в среде с $\varkappa>0$ начальная точка $A$ является узлом с выходящими линиями, точка $B$ на отрезке $Q E$ - седло, и имеются две интегральные кривые из $A$ в $B$, т. е. две вязкие структуры для этой ударной волны.

На рис. 2.2 а представлена картина линий уровня функции $Z\left(u_{1}, u_{2}\right)$ (пунктирная линия - ударная адиабата), на рис. $2.2 b$ изображена соответствующая диаграмма эволюционности (рис. $2.2 b$ ) для значений параметров $U_{1}=U_{2}=1$, $f=1, g=0.03, \varkappa=-0.5, W=2.6$. Для медленной ударной волны $A \rightarrow B$ (типа $D L$ ) при $\varkappa<0$ точка $A$ - седло, точка $B$ - узел с входящими линиями. Имеются две интегральные кривые из $A$ в $B$ и две возможных структуры этого разрыва.

Было показано [33], [34], что и все другие априорно эволюционные квазипоперечные ударные волны обладают вязкой стационарной структурой и, таким образом, имеют право на существование, а также что нет других волн, 

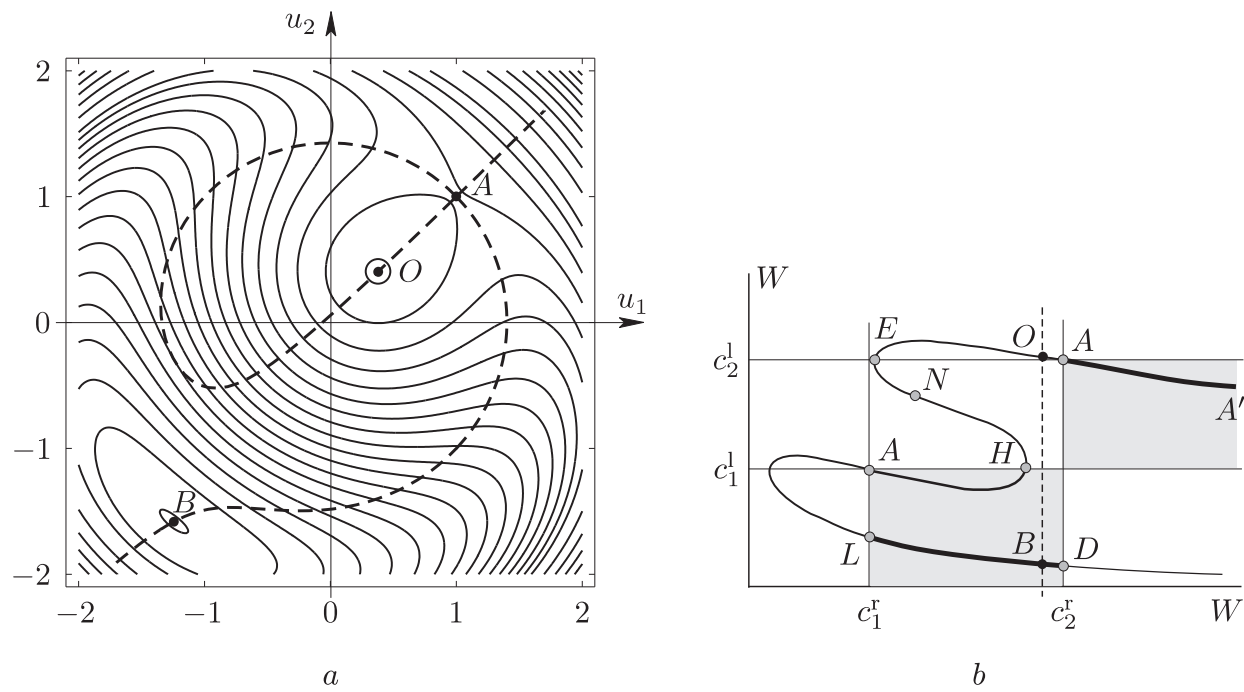

Рис. 2.2. Структура квазипоперечных ударных волн в средах с $\varkappa<0$

обладающих структурой. Таким образом, проверка ударных волн на наличие структуры не привела к исключению каких-либо ударных волн как не имеющих структуры и, следовательно, не решила вопроса о выборе решения автомодельных задач в области неединственности.

\section{2. Одномерные нестационарные решения. Описание решений} в условиях неединственности автомодельных асимптотик. Если рассматривать упругую среду как предел вязко-упругой при $\mu \rightarrow 0$, то, изучая асимптотические формы решений, можно надеяться получить ответ о выборе однозначного решения задач, решение которых неоднозначно при отсутствии вязкости. Ряд нестационарных неавтомодельных задач для вязко-упругой среды был решен в [35] для сред с $\varkappa>0$ и в [36] для сред с $\varkappa<0$. Ограничимся здесь изложением результатов только для $\varkappa>0$. Для этих задач рассмотренные выше автомодельные решения представляют асимптотики при $t \rightarrow \infty$. Для системы уравнений (2.1) численно решалась следующая начально-краевая задача:

$$
\begin{gathered}
u_{\alpha}=U_{\alpha} \quad \text { при } t=0, x \geqslant 0, \\
u_{\alpha}= \begin{cases}u_{\alpha}^{0}(t) & \text { при } x=0,0 \leqslant t \leqslant \tau, \\
u_{\alpha}^{*} & \text { при } x=0, \tau<t, \\
u_{\alpha}^{*}, \tau=\text { const. }\end{cases}
\end{gathered}
$$

Рассмотрим большие значения времени $t \gg \tau$, когда характерный линейный размер задачи велик. В этом случае можно ожидать, что эффект вязкости и изменения граничных условий внутри интервала $\tau$ слабеет и решение будет стремиться к определенному, хотя и изначально неизвестному автомодельному решению задачи без вязкости. Это решение может зависеть от функций, определяющих граничные значения $u_{\alpha}$ при $0 \leqslant t \leqslant \tau$. 
Ниже приводятся результаты численного решения ряда начально-краевых задач для уравнений (2.1) с вязкими членами, для которых рассмотренные в $\S 1$ автомодельные решения могут представлять асимптотики при $t \rightarrow \infty$. Коэффициент вязкости $\mu$ выбирался таким образом, чтобы вязкость физическая, описываемая членом $\mu \partial^{2} u_{\alpha} / \partial x^{2}$, была больше, чем вязкость, которая возникает при решении разностных уравнений, аппроксимирующих дифференциальную задачу.

Для одного из вариантов расчета $(\tau=0)$ начальные условия $(t=0)$ и правое граничное условие $(t>0, x=l)$ взяты в виде $u_{1}=1, u_{2}=1$ (точка $A$ на рис. 1.4$)$, на левой границе $(t>0, x=0)$ принято $u_{1}^{*}=-1.05, u_{2}^{*}=-0.455$ (точка $B$ принадлежит эволюционному отрезку $Q E$ ударной адиабаты, соответствующей начальной точке $A$, и области неединственности решения); $g=0.03$, $\varkappa=0.5, \mu=0.024$. В процессе расчета сформировалась структура ударной волны типа $Q E$. Результаты численного расчета для фиксированного (большого) момента времени приведены на рис. 2.3. На рис. 2.3 а представлен график

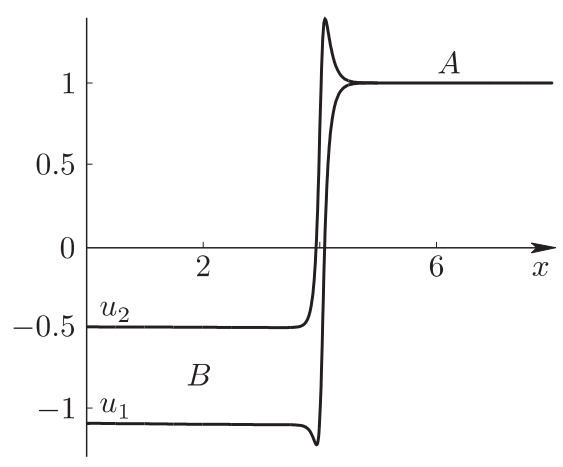

$a$

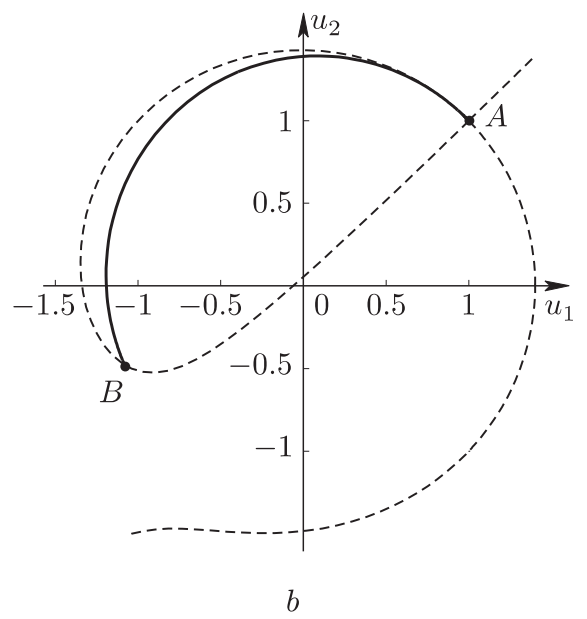

$b$

Рис. 2.3. Асимптотика автомодельного решения первого типа в средах c $\varkappa>0$

решения в зависимости от координаты $x$. Точкам $A$ и $B$ соответствуют участки оси $x$, на которых величины $u_{1}, u_{2}$ принимают постоянные значения. Было проверено, что эти постоянные значения, представляющие состояния перед и за ударной волной $A \rightarrow B$ с принятой степенью точности удовлетворяют уравнению соответствующей ударной адиабаты. Следует отметить, что внутри структуры ударной волны $A \rightarrow B$ величина $u_{2}$ имеет локальный максимум (рис. $2.3 a$ ). Это согласуется с исследованием структуры (п. 2.1). На рис. $2.3 b$ сплошной линией представлен график решения на плоскости $\left(u_{1}, u_{2}\right)$, представляющий структуру ударной волны $A \rightarrow B$, пунктирной линией на этом рисунке проведена ударная адиабата для выбранных параметров.

В результате серий расчетов с различными функциями $u_{\alpha}^{0}(t)$ и $\tau \neq 0$ показано, что в области неединственности (заштрихованной на рис. 1.4) на плоскости $\left(u_{1}^{*}, u_{2}^{*}\right)$ при больших $t$ обычно реализуется решение первого типа, содер- 
жащее ударную волну типа $Q E$. Получить решения второго типа, содержащие "сложную" ударную волну, оказалось возможным только в том случае, когда в течение некоторого временного интервала $\tau_{1}$ граничные условия при $x=0$ таковы, что точка с координатами $u_{\alpha}^{*}(t)$ находится в области, где существует решение только второго типа, т. е. в области, расположенной ниже заштрихованной области неединственности (рис. 1.4). При этом в течение времени $\tau_{1}$ развивается решение второго типа. Если $\tau_{1}$ достаточно велико, а затем граничные условия подвергаются изменению таким образом, что точка, задающая граничные условия, переходит при $t>\tau_{1}$ в область неединственности, то возмущения, возникающие при изменении граничных условий, уже не могут изменить тип решения, начавшего формироваться при $t<\tau_{1}$, и оно стремится к автомодельному решению второго типа в области неединственности.

Результаты счета, представляющие решение, выходящее на автомодельную асимптотику второго типа, представлены на рис. 2.4. На рис. 2.4 а приведены графики решения для двух последовательных моментов времени $t_{1}$ (сплошная линия) и $t_{2}$ (пунктирная линия) $\left(t_{1}<t_{2}\right)$. Точкам $A$ и $M$ на рис. $2.4 b$

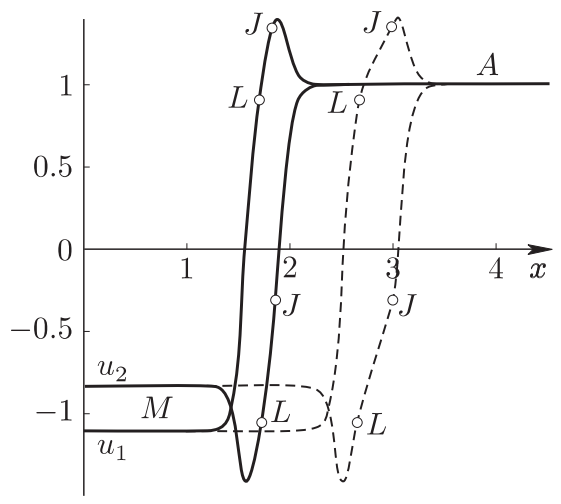

$a$

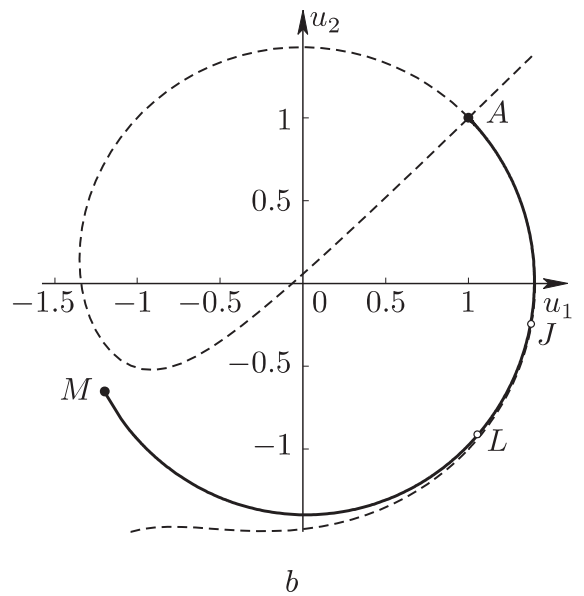

$b$

Рис. 2.4. Асимптотика автомодельного решения второго типа в средах с $\varkappa>0$

соответствуют участки оси $x$, на которых величины $u_{1}, u_{2}$ принимают постоянные значения. Точка $M$ принадлежит области неединственности (рис. 1.4). На рис. 2.4 а можно выделить последовательность простых и ударных волн, соответствующих сложной волне, входящей в состав автомодельного решения второго типа: быстрая ударная волна Жуге - скачок из точки $A$ в точку $J$ (точка $J$ - точка Жуге по состоянию за скачком), затем идет отрезок интегральной кривой $J L$ быстрой волны Римана, быстрая ударная волна Жуге - скачок из $L$ в $M$ (условие Жуге выполнено в точке $L$ ). В структуре скачка из начальной точки $A$ в точку $J$ величина $u_{1}$ имеет локальный максимум, в структуре скачка из точки $L$ в $M$ величина $u_{2}$ имеет локальный минимум. Это согласуется с видом интегральных кривых (п. 2.1). Сравнение графиков в два последовательных момента времени показывает, что участок $J L$, соответствующий 
простой волне, расширяется с течением времени, а участки, соответствующие структурам скачков $A \rightarrow J$ и $L \rightarrow M$, не изменяются. Таким образом, следует подчеркнуть, что структура “сложной” волны, входящей в состав автомодельного решения второго типа, расширяется с увеличением времени. Кривая $u_{1}(x), u_{2}(x)$ представлена на рис. $2.4 b$ на плоскости $\left(u_{1}, u_{2}\right)$ сплошной линией. Пунктирной линией на этом рисунке проведена ударная адиабата для начальной точки $A\left(U_{1}, U_{2}\right)$.

Полученные многочисленные решения показывают, что решение неавтомодельной задачи может стремиться при $t \rightarrow \infty$ к разным решениям предельной автомодельной задачи (если это решение неединственно), в зависимости от деталей постановки начальных и граничных условий, соответствующих ограниченным значениям времени. Автомодельные асимптотики, как показывают результаты расчетов, устойчивы. Это означает, что в пространстве функций $u_{\alpha}^{0}(t)$ у каждой из асимптотик есть область притяжения. Насколько можно судить, область притяжения у автомодельного решения первого типа в каком-то смысле больше, чем у автомодельного решения второго типа.

Разделить явным образом все начальные и граничные условия на два класса, соответствующих различному асимптотическому поведению при $t \rightarrow \infty$, практически невозможно. Однако, если ограничиться рассмотрением постановок задач, в которые входят непрерывные функции с ограниченными производными, то при $\mu \rightarrow 0$ для однозначного продолжения решений по времени нужно уметь решать задачи о взаимодействии двух ударных волн или ударной волны и малого возмущения (столкновение большего числа волн маловероятно). При этом, конечно, интерес представляют случаи, когда соответствующее автомодельное решение неединственно.

\section{3. Взаимодействие нелинейных волн в слабоанизотропной среде.} Изучению процесса взаимодействия ударных волн посвящены работы [35]-[38]. В этом пункте рассмотрены: пример 2.1, демонстрирующий решение задачи о взаимодействии волн, движущихся в одном направлении, и примеры 2.2, 2.3, представляющие решения задач о столкновении двух ударных волн. Задачи о взаимодействии ударных волн наиболее интересны в том случае, когда образовавшемуся разрыву соответствует не единственное решение автомодельной задачи.

ПримеР 2.1. В случае $\varkappa>0$ в задаче о взаимодействии двух ударных волн, одна из которых догоняет другую, была сформирована структура медленной ударной волны, соответствующей эволюционному скачку из точки $A$ в точку $A_{1}$ (рис. 2.5), и структура догоняющей ее быстрой ударной волны, соответствующей эволюционному скачку из точки $A_{1}$ в точку $B$ (рис. 2.5). Точка $B$ расположена на ударной адиабате, проведенной из точки $A_{1}$ как начальной, и принадлежит области неединственности решения автомодельной задачи, соответствующей точке $A$ как начальной. Счет показал, что в результате взаимодействия этих ударных волн образуются две другие ударные волны: впереди идет быстрая $A \rightarrow Q_{1}$, за ней медленная $Q_{1} \rightarrow B$; таким образом, решение выходит на автомодельную асимптотику, соответствующую решению первого типа автомодельной задачи о распаде произвольного начального разрыва. 


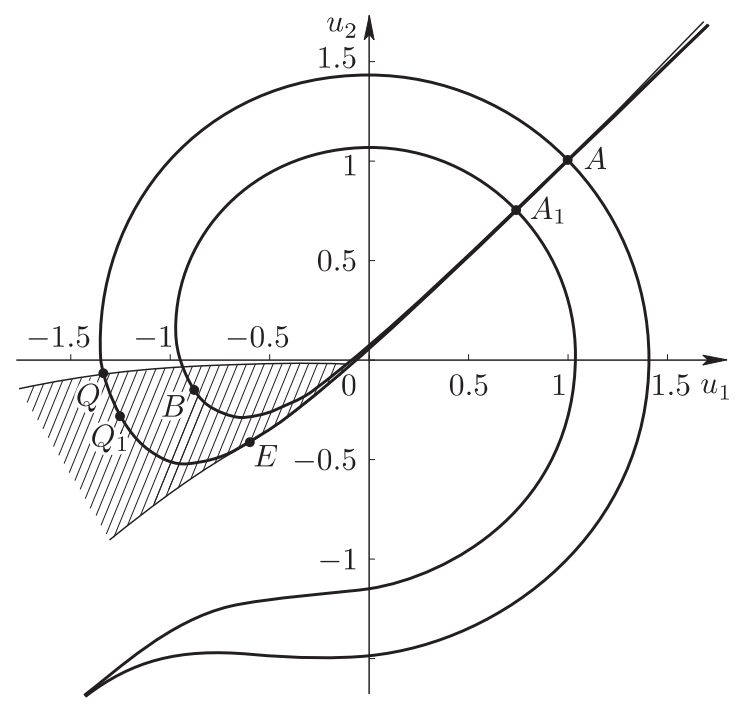

Рис. 2.5. Пример $2.1(\varkappa>0)$

Также была решена задача о взаимодействии ударной быстрой волны, соответствующей эволюционному скачку типа $Q E$ из точки $A$ в точку $Q_{1}$ (или любую другую точку эволюционного участка $Q E)$, и малого финитного возмущения. Малое возмущение было сформировано перед быстрой ударной волной. Проведенные расчеты показали, что в результате взаимодействия ударной волны и малого возмущения образуется одна ударная волна типа $Q E$.

ПримеР 2.2. Рассматривалась задача о взаимодействии двух ударных волн, движущихся одна навстречу другой. Для этого исследования должна, вообще говоря, использоваться полная система уравнений вязко-упругости, описывающая волны, движущиеся в противоположных направлениях. Однако в случае малой нелинейности задачу можно существенно упростить и необходимые для исследования расчеты проводить, используя упрощенную систему уравнений (2.1).

Очевидно, что встречные волны взаимодействуют в течение ограниченного промежутка времени $\tau$, определяемого шириной структуры этих волн и их скоростью. Поэтому влиянием слабой нелинейности и слабой анизотропии в период взаимодействия встречных волн можно пренебречь, так что волны за время $\tau$ ведут себя как линейные, т. е. не меняют своей формы, и лишь потом, при существенно больших временах, происходит их изменение за счет действия малых нелинейных членов. Причиной этих изменений является то, что каждая из волн после времени $\tau$ начинает двигаться по измененному фону (рис. 2.6). Это приводит к нарушению соотношений на разрывах и к распаду волн, возникших после встречи двух исходных волн.

Рассмотрим следующую задачу о взаимодействии ударных волн, движущихся одна навстречу другой в среде с $\varkappa>0$. На рис. 2.7 сплошной линией изображена ударная адиабата из начальной точки $A_{1}(0.76,1.22)$, пунктирной линией 


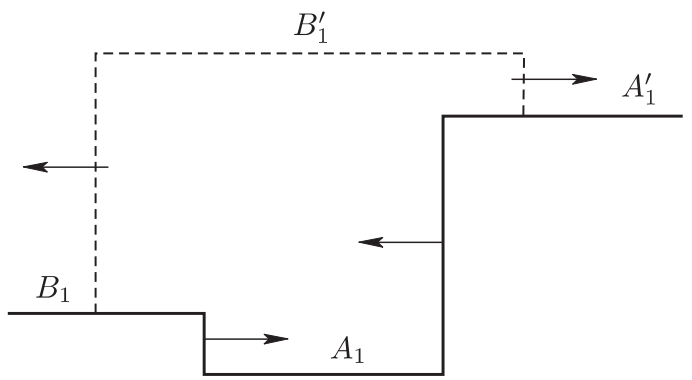

Рис. 2.6. Задача о взаимодействии двух ударных волн, движущихся навстречу друг другу

изображена ударная адиабата из начальной точки $A_{1}^{\prime}(1,1)$, лежащей на ударной адиабате из начальной точки $A_{1}(f=1, g=0.05, \varkappa=0.5)$. С целью исследовать процесс взаимодействия встречных ударных волн была сформирована структура быстрой ударной волны, движущейся налево и соответствующей скачку из точки $A_{1}$ в точку $A_{1}^{\prime}$, и структура движущейся ей навстречу быстрой ударной волны, соответствующей скачку из точки $A_{1}$ в точку $B_{1}(-1.28,-0.2)$.

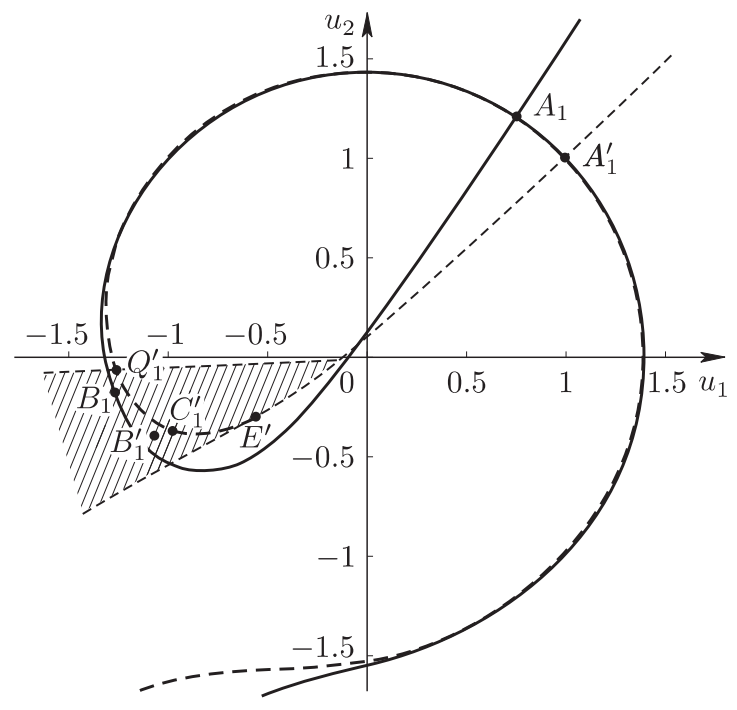

Рис. 2.7. Пример $2.2(\varkappa>0)$

В результате описанного выше линейного взаимодействия структур ударных волн $A_{1} \rightarrow A_{1}^{\prime}$ и $A_{1} \rightarrow B_{1}$, движущихся навстречу, вправо будет распространяться возмущение $A_{1}^{\prime} \rightarrow B_{1}^{\prime}$, влево - возмущение $B_{1} \rightarrow B_{1}^{\prime}$ (рис. 2.6). Точка $B_{1}^{\prime}$ определяется из условия, что разность значений величин $u_{1}, u_{2}$ за и перед возмущением $A_{1}^{\prime} \rightarrow B_{1}^{\prime}\left(\Delta u_{1}\right.$ и $\left.\Delta u_{2}\right)$ равна разности этих же величин за и перед скачком $A_{1} \rightarrow B_{1}$. Согласно предыдущему, можно считать, что скачок $A_{1} \rightarrow B_{1}$ после взаимодействия со скачком $A_{1} \rightarrow A_{1}^{\prime}$ сохранил свою структуру, 
но распространяется по новому начальному состоянию среды, соответствующему точке $A_{1}^{\prime}$.

Исследуем эволюцию возмущения $A_{1}^{\prime} \rightarrow B_{1}^{\prime}$. Из точки $A_{1}^{\prime}$ как начальной проведена ударная адиабата, изображенная на рис. 2.7 пунктирной линией. На рис. 2.7 заштрихована область неединственности решения задачи для точки $A_{1}^{\prime}$ как начальной. Точка $B_{1}^{\prime}$ принадлежит этой области неединственности.

Для исследования эволюции возмущения $A_{1}^{\prime} \rightarrow B_{1}^{\prime}$ была поставлена следующая начально-краевая задача для системы уравнений (2.1). В качестве начальных условий $(t=0)$ задана структура скачка $A_{1} \rightarrow B_{1}$, распространяющегося по состоянию $A_{1}^{\prime}$. Правое граничное условие $(t>0, x=l)$ взято в виде $U_{1}=U_{2}=1$ (точка $A_{1}^{\prime}$ на рис. 2.7). На левой границе $(t>0, x=0$ ) принято $u_{1}^{*}=-1.04, u_{2}^{*}=-0.42$ (точка $B_{1}^{\prime}$ на этом же рисунке). Численное решение поставленной начально-краевой задачи при достаточно больших временах представляет собой последовательность распространяющихся направо быстрой ударной волны $A_{1}^{\prime} \rightarrow C_{1}^{\prime}$ и медленной волны Римана $C_{1}^{\prime} \rightarrow B_{1}^{\prime}$, т. е. автомодельную асимптотику решения первого типа.

Влево будет распространяться возмущение $B_{1} \rightarrow B_{1}^{\prime}$. Точка $B_{1}^{\prime}$ не является точкой области неединственности решения автомодельной задачи для начальной точки $B_{1}$. Поэтому система волн, движущихся после взаимодействия налево, определяется однозначно.

ПримеР 2.3. При тех же значениях параметров $(f=1, g=0.05, \varkappa=0.5)$, что и в примере 2.2 , рассмотрено взаимодействие медленной ударной волны $A_{2} \rightarrow A_{2}^{\prime}$, движущейся налево, и быстрой ударной волны $A_{2} \rightarrow B_{2}$, движущейся направо (начальные и конечные состояния заданы координатами точек $\left.A_{2}(1,1), A_{2}^{\prime}(0.86,0.87), B_{2}(-1.1,-0.3)\right)$. На рис. 2.8 сплошной линией изобра-

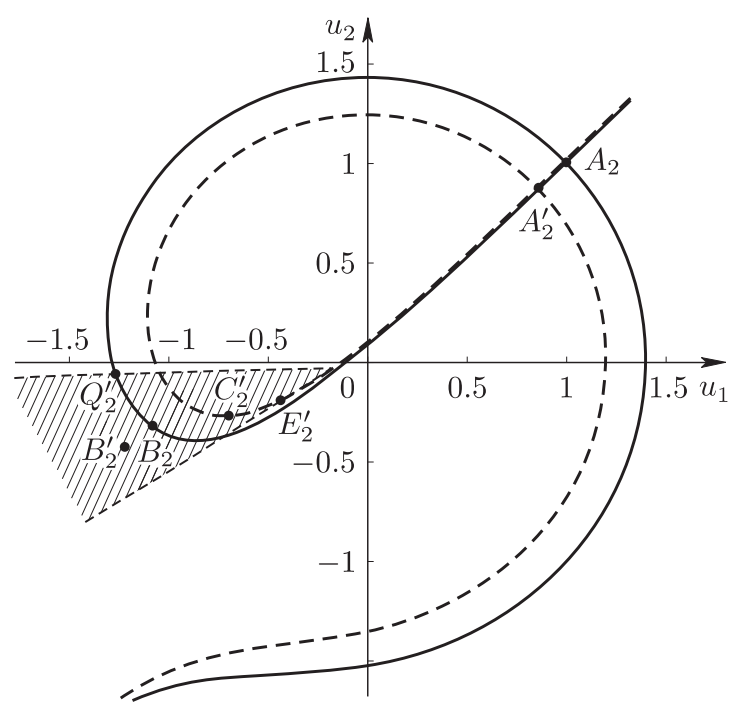

Рис. 2.8. Пример $2.3(\varkappa>0)$

жена ударная адиабата, проведенная из точки $A_{2}$ как начальной, пунктирной линией - ударная адиабата из точки $A_{2}^{\prime}$ как начальной. 
В результате линейного взаимодействия встречных ударных волн $A_{2} \rightarrow A_{2}^{\prime}$ и $A_{2} \rightarrow B_{2}$ направо будет распространяться возмущение $A_{2}^{\prime} \rightarrow B_{2}^{\prime}$, налево возмущение $B_{2} \rightarrow B_{2}^{\prime}$. Точка $B_{2}^{\prime}$ принадлежит заштрихованной на рис. 2.8 области неединственности решения автомодельной задачи, соответствующей точке $A_{2}^{\prime}$ как начальной.

Для системы уравнений (2.1) численно решалась начально-краевая задача с начальными данными, соответствующими структуре скачка $A_{2} \rightarrow B_{2}$, pacпространяющегося по состоянию $A_{2}^{\prime}$, и следующими граничными условиями: на правой границе (при $t>0, x=l) U_{1}=0.86, U_{2}=0.87$, что соответствует точке $A_{2}^{\prime}$, и на левой границе (при $\left.t>0, x=0\right) u_{1}^{*}=-1.24, u_{2}^{*}=-0.43$, что соответствует точке $B_{2}^{\prime}$. Счет показал, что при достаточно больших временах решение состоит из быстрой ударной волны $A_{2}^{\prime} \rightarrow C_{2}^{\prime}$ и медленной простой волны $C_{2}^{\prime} \rightarrow B_{2}^{\prime}$, т. е. выходит на автомодельную асимптотику первого типа.

Система волн, движущихся после взаимодействия встречных ударных волн $A_{2} \rightarrow A_{2}^{\prime}$ и $A_{2} \rightarrow B_{2}$ налево, определяется однозначно, так как точка $B_{2}^{\prime}$ не принадлежит области неединственности решения автомодельной задачи для точки $B_{2}$ как начальной.

Исследование взаимодействия двух ударных волн, движущихся навстречу друг другу, показало, что так же, как в случае волн, движущихся в одну сторону, решение выходит на автомодельную асимптотику, соответствующую системе волн автомодельного решения первого типа. Этот результат позволяет сделать вывод, что в случае задач с медленно меняющимися начальными и граничными условиями процессы взаимодействия возникающих в решении ударных волн будут происходить, по-видимому, всегда так, как если бы второго автомодельного решения не существовало.

Таким образом, на основании расчетов, изложенных в пп. 2.2, 2.3, можно заключить, что автомодельные асимптотики первого типа (последовательность быстрой ударной волны и медленной ударной или простой волны) образуются более устойчиво (обладают в некотором смысле большей областью притяжения), чем автомодельные асимптотики второго типа.

\section{4. Исследование устойчивости структуры ударных волн в вяз- ко-упругой среде при взаимодействии с одномерными неоднороднос-} тями. Рассмотрим теперь вопрос о возможности выделения единственного решения путем исключения некоторых типов разрывов как неустойчивых. Для случая $\varkappa>0$, который рассматривается далее, наибольшее подозрение, как уже говорилось, вызывает разрыв $A \rightarrow B \in Q E$ (разрыв типа $Q E$ ), входящий в решение первого типа. Действительно, как отмечалось в п. 2.2, изменение величин в этой ударной волне совпадает с изменением величин в некоторой системе волн, движущихся в ту же сторону с различными скоростями и составляющих автомодельное решение второго типа. Таким образом, ударная волна типа $Q E$ может распасться на систему волн, и если такое событие произойдет, то эти волны, двигаясь с разными скоростями, уже никогда не соберутся в исходную ударную волну. Ударные волны типа $Q E$ являются метастабильными объектами, и часто предполагается, что ударные волны, распад которых допускается системой гиперболических уравнений, не присутствуют в решениях 
задач [39]. Если принять такую точку зрения, то решение рассматриваемых задач об упругих волнах в случае $\varkappa>0$ окажется всегда единственным и в области, которая считается областью неединственности, будет только второго типа. Однако метастабильность есть неустойчивость по отношению к конечным возмущениям, причем известно, что, например, метастабильные состояния могут реализовываться и достаточно долго существовать. Возможность реализации метастабильных объектов определяется соотношением между необходимыми для их разрушения и имеющимися в наличии возмущениями.

Решить вопрос, распадается или не распадается ударная волна, невозможно, пока она рассматривается как поверхность нулевой толщины. Ниже приводятся результаты численных экспериментов по изучению взаимодействия метастабильной волны (типа $Q E$ ) с локальными (финитными) неоднородностями фона в предположении, что среда является вязко-упругой средой Кельвина-Фойхта. Эта проблема представляет интерес ввиду того, что фон, по которому распространяются волны, в реальности всегда слегка неоднороден, причем неоднородность может иметь случайный характер. Возникает вопрос, насколько велики должны быть неоднородности фона, чтобы произошел распад ударной волны на систему волн. В [40] предполагалось, что задающие свойства фона коэффициенты уравнений, описывающих упругие волны, являются функциями координаты $x$, в направлении которой происходит распространение плоских волн, причем в конкретных расчетах предполагалось, что фон всюду однородный, за исключением некоторого отрезка оси $x(a<x<b)$, где он также однороден, но характеризуется другими параметрами. Изучается, как происходит взаимодействие с такой неоднородностью бегущих волн, приходящих из области $x<a$ и соответствующих в невязком случае исследуемым ударным волнам типа $Q E$.

На границах отрезка $[a, b]$ предполагаются выполненными условия непрерывности перемещений и напряжений. Эти условия задают законы отражения и преломления волн на этих границах. Очевидно, что когда закончится нестационарный процесс, связанный с прохождением волной отрезка $[a, b]$, внутри отрезка и на его границах установятся некоторые новые постоянные напряжения. Поэтому задача о возникающих в области $x>b$ волнах должна иметь при $t \rightarrow \infty$ автомодельную невязкую асимптотику в переменных $x / t, t$. Эта асимптотика при некоторых условиях может быть первого типа и содержать волну типа $Q E$. В других случаях асимптотика может быть второго типа, в которой волна типа $Q E$ не содержится и заменяется системой других волн. В последнем случае будем говорить о распаде волны типа $Q E$ при прохождении неоднородности на отрезке $[a, b]$. Целью проведенного исследования является обнаружение и оценка пороговых свойств неоднородностей среды, разрушающих исходную волну.

В рассматриваемой ниже задаче влияние малых членов должно учитываться в тех случаях, когда они действуют в течение достаточно большого времени. При взаимодействии волн с концами отрезка $[a, b]$ достаточно учесть первый член в выражении (1.4), а вязкими и нелинейными членами в уравнениях можно пренебречь. Для простоты в дальнейшем рассматриваются случаи, когда эффектами отражения волн от границ отрезка $[a, b]$ можно также пренебречь, для чего достаточно потребовать постоянства $\rho_{0}$ и $f$ всюду. В связи с этим 
оказалось удобным изучать волны, распространяющиеся только в одном направлении (в положительном направлении оси $x$ ).

Для описания волновых процессов, как и прежде, используется система уравнений (2.1), а упругий потенциал имеет вид (1.4). Выражение (1.4) справедливо в специальной системе декартовых координат, в которой направления осей $x_{1}$ и $x_{2}$ определяются свойствами среды. В дальнейшем будут использоваться эти специальные координаты. Поэтому на выделенном отрезке $[a, b]$ могут быть изменены по отношению к окружающей среде не только упругие коэффициенты, но и ориентация указанных осей, что приведет к ортогональным преобразованиям переменных $u_{1}, u_{2}$, представляющих собой поворот осей $u_{1}, u_{2}$ сначала на некоторый угол $\theta$ при $x=a$, а затем на угол $-\theta$ при $x=b$.

Вследствие предполагаемой малой нелинейности волн и конечной скорости их распространения можно считать, что изменение свойств среды при пересечении волной границ отрезка $[a, b]$ можно заменить мгновенным изменением величин $g, \mu, \theta$ в структуре волны, считая их ступенчатыми функциями времени $t$, скачкообразно меняющимися дважды. При численных экспериментах определяется наименьший интервал времени такой, что некоторое заданное изменение параметров среды в этом интервале приводит к распаду изучаемой ударной волны.

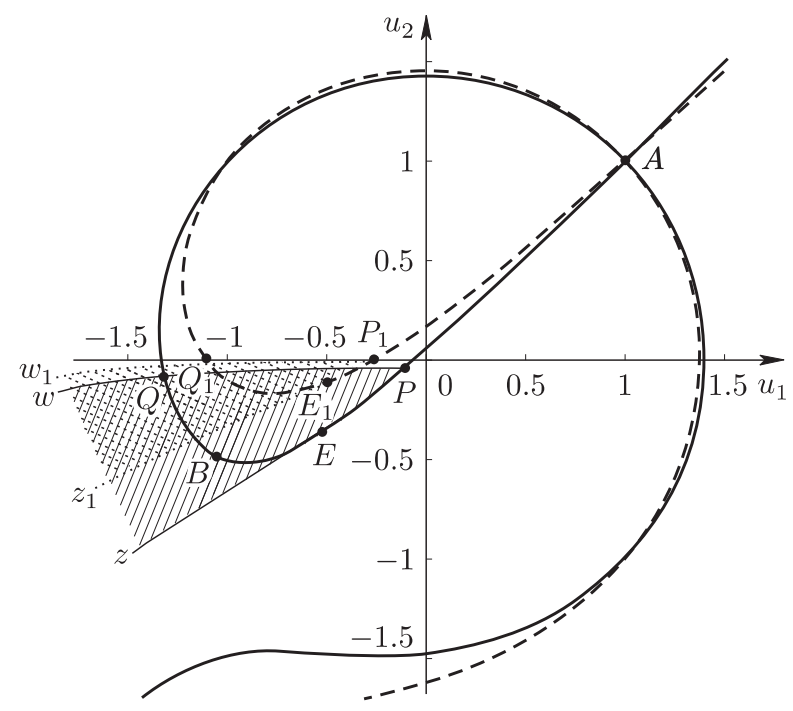

Рис. 2.9. Ударные адиабаты для параметров $g=0.03$ (сплошная линия), $g=0.1$ (пунктирная линия)

При проведении расчетов сначала формировалось решение в виде бегущей волны $A \rightarrow B$ такой, что точка $B$ принадлежит отрезку $Q E$ ударной адиабаты, соответствующей начальной точке $A$ (рис. 2.9) (точка $A$ имела координаты $U_{1}=1, U_{2}=1$, точка $B$ - координаты $u_{1}^{*}=-1.05, u_{2}^{*}=-0.455 ; g=g_{1}=0.03$; $\varkappa=0.5 ; \mu=0.024)$. На рис. 2.9 сплошной линией изображена ударная адиабата для этих параметров и сплошной штриховкой отмечена область неединственности (кривые $P Q w, P E z)$. Точка $B$ принадлежит области неединственности 
для начального состояния, соответствующего точке $A$. Параметр $f$ выбирался (используя преобразование Галилея) таким образом, чтобы волна $A \rightarrow B$ все время оставалась в расчетной области.

В первой серии расчетов проводилось изменение значения параметра анизотропии $g$ на промежутке времени $t_{a}<t<t_{b}$, соответствующее прохождению волной участка оси $x(a<x<b)$. Значения остальных параметров $\varkappa, \mu, f$ не изменялись. При $0<t<t_{a}$ и $t>t_{b}$ значение $g$ считалось равным $g_{1}=0.03$, а на выделенном промежутке времени $t_{a}<t<t_{b}$ значение параметра $g$ увеличивалось и принимало значение $g_{2}=0.1$.

Ударная адиабата для измененного параметра $g_{2}=0.1$ изображена на рис. 2.9 пунктирной линией. В этом случае изменяется геометрия области неединственности, которая ограничена пунктирными линиями $P_{1} Q_{1} w_{1}, P_{1} E_{1} z_{1}$. При таком изменении параметра $g$ точка $B$ принадлежит области, в которой при $q_{2}=0.1$ решение единственно и является решением второго типа. Поэтому при $t_{a}<t<t_{b}$ сформированная вне этого отрезка структура ударной волны $A \rightarrow B$ начинает распадаться на систему волн автомодельного решения второго типа. Эта система волн расширяется с увеличением времени (пп. 2.1, 2.2), поэтому чем больше длина отрезка $\left[t_{a}, t_{b}\right]$, тем будет больше ширина волнового пакета.

Проведена также серия расчетов для случая, когда на отрезке времени $\left[t_{a}, t_{b}\right]$ изменялся параметр $\theta$, а остальные параметры среды не изменялись. Аналогично предыдущей серии расчетов, к моменту времени $t_{a}$ было сформировано решение, представляющее структуру быстрой ударной волны $A_{1} \rightarrow B_{1}$ (типа $Q E$ ) (см. рис. 2.10). Затем при $t_{a}<t<t_{b}$ изменялось направление осей $u_{1}$

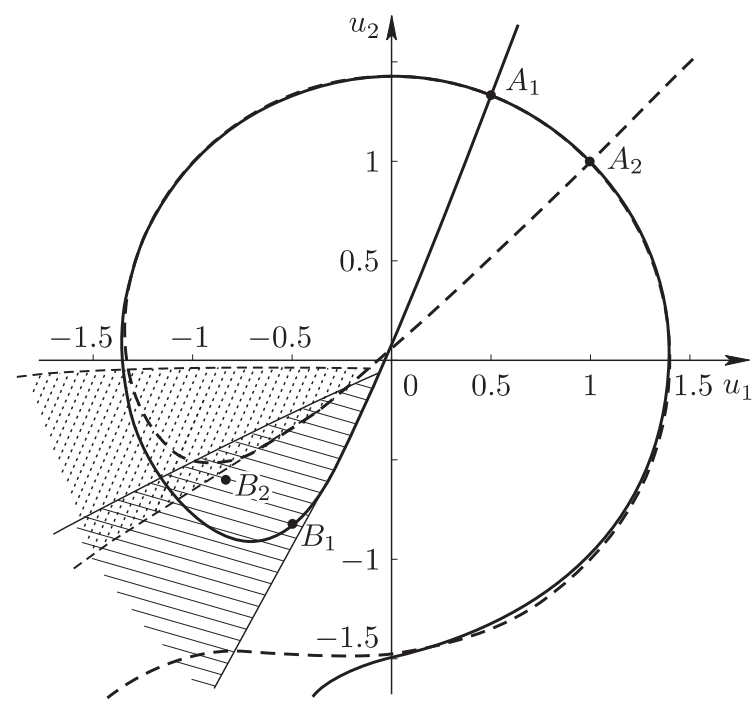

Рис. 2.10. Ударные адиабаты для начальных точек $A_{1}(0.5,1.33)$ (сплошная линия), $A_{2}(1,1)$ (пунктирная линия)

и $u_{2}$ на угол $\theta$ таким образом, что в новых осях точка $A_{1}$ перешла в точку $A_{2}$, точка $B_{1}-$ в точку $B_{2}$. На рис. 2.10 сплошной линией изображена ударная 
адиабата из начальной точки $A_{1}$ и сплошными линиями заштрихована соответствующая область неединственности решений задачи, пунктирной линией проведена ударная адиабата из начальной точки $A_{2}$ и пунктирной штриховкой отмечена соответствующая область неединственности. Угол поворота осей $\theta$ выбирался таким образом, чтобы точка $B_{2}$ принадлежала области, в которой поставленная начально-краевая задача, соответствующая точкам $A_{2}, B_{2}$, для системы уравнений (2.1) имела единственное решение второго типа. В момент времени $t=t_{b}$ оси $u_{1}, u_{2}$ поворачивались на угол $-\theta$, т. е. занимали исходное начальное положение, при этом точка $A_{2}$ возвращалась в точку $A_{1}$, а точка $B_{2}-$ в точку $B_{1}$.

В этих двух сериях расчетов показано, что распад метастабильной волны происходил только тогда, когда изменения $g$ или $\theta$ были такими, что в результате этих изменений точка $B$, представляющая состояние за невозмущенной метастабильной волной, попадала в область, в которой существует только решение второго типа. При этом начинало формироваться решение второго типа. Если разность $\tau=t_{b}-t_{a}$ оказывалась достаточно большой, то волны, его составляющие, расходились по оси $x$ и далее при $t>t_{b}$ продолжали представлять решение второго типа. Если же величина $\tau$ была недостаточно большой, то из решения, возмущенного изменением $g$ или $\theta$, вновь вырабатывалось при $t>t_{b}$ решение первого типа, представляемое структурой метастабильной ударной волны.

Численные эксперименты по определению величины времени $\tau=t_{b}-t_{a}$, которое необходимо для распада метастабильных волн, показали [40], что величина $\tau$ определяется следующим условием: для распада необходимо, чтобы общая протяженность системы волн, образовавшихся на участке неоднородности и движущихся с различными скоростями, была существенно больше ширины структуры метастабильной ударной волны (типа $Q E$ ) вне области неоднородности. Если оценить ширину ударной волны $l$ исходя из равенства нелинейного и вязкого членов как $l \sim \mu / u^{2}$, а скорость расхождения волн, возникших в результате распада разрыва, как $\varkappa u^{2}$, то условие необратимого распада можно записать в виде $\tau>\mu /\left(\varkappa u^{4}\right)$, где $\tau=t_{a}-t_{b}, u$ - характерная величина $u_{\alpha}$.

Численно также были решены начально-краевые задачи для системы уравнений $(2.1)$, когда на выделенном отрезке $\left[t_{a}, t_{b}\right]$ изменялась величина коэффициента кинематической вязкости $\mu$, а остальные коэффициенты уравнений $(2.1)$ не изменялись. На отрезке $\left[t_{a}, t_{b}\right]$ значение коэффициента вязкости $\mu$ увеличивалось и как следствие этого ширина структуры ударной волны типа $Q E$ на этом отрезке увеличивалась, но характерное изменение величин $u_{1}$ и $u_{2}$ внутри структуры сохранялось. Последующее возвращение коэффициента вязкости на границе $t=t_{b}$ к первоначальному значению приводило к тому, что ширина структуры ударной волны постепенно уменьшалась и при увеличении времени $t$ метастабильная ударная волна восстанавливалась независимо от величины $\tau=t_{b}-t_{a}$.

Изложенные результаты, касающиеся взаимодействия метастабильной ударной волны с неоднородностями фона, находятся в соответствии с результатами пп. 2.2, 2.3 о формировании решений первого и второго типов и взаимодействии нелинейных волн между собой. Для распада метастабильной волны необходимо не просто возмутить ее структуру (как, например, с помощью изменения 
вязкости), но нужно добиться путем создания специальных возмущений конечной амплитуды формирования решения второго типа до стадии, не оставляющей возможности сохранения решения первого типа. Вместе с тем, как следует из изложенного, время $\tau$, необходимое для разрушения структуры метастабильной ударной волны специальными нелинейными возмущениями фона, не может быть меньше величины $\mu /\left(\varkappa u^{4}\right)$, указанной выше. Таким образом, метастабильная ударная волна проявляет большую устойчивость по отношению к одномерным возмущениям.

\section{5. Устойчивость к двумерным возмущениям метастабильной} ударной волны в вязко-упругой среде. В предыдущем пункте было показано, что взаимодействие метастабильной ударной волны с достаточно большими и протяженными неоднородностями фона может приводить к необратимому распаду метастабильной ударной волны, так что после взаимодействия образуется система волн, соответствующая упомянутому выше решению второго типа задачи о распаде разрыва. Несмотря на довольно жесткие условия, необходимые для того, чтобы произошел распад метастабильной ударной волны, остается возможность считать, что при движении по случайному фону метастабильная ударная волна рано или поздно наткнется на достаточно большое возмущение, которое ее разрушит. В связи с этим представляет интерес изучение в той же постановке, что и ранее, взаимодействия метастабильной ударной волны с неодномерными возмущениями.

В этом пункте обсуждаются результаты численного решения двумерных вязко-упругих задач о взаимодействии вязкой структуры метастабильной ударной волны с достаточно медленно меняющимися периодическими по тангенциальной координате возмущениями [41]. Результаты решения этих задач показывают, что если после взаимодействия остается достаточно большой участок невозмущенной метастабильной ударной волны, то ее распад на систему волн на другом участке волн носит обратимый характер, т. е. с течением времени восстанавливается решение, соответствующее исходной метастабильной ударной волне.

Будем использовать упрощенную систему уравнений [42] для описания двумерных нестационарных движений вязко-упругой среды, когда все искомые величины зависят от времени $t$, лагранжевой координаты $x$ и слабо зависят от лагранжевой координаты $y$ (в начальном состоянии $x$ и $y$ совпадают соответственно с декартовыми координатами $x_{3}$ и $\left.x_{2}\right)$ :

$$
\frac{\partial u_{\alpha}}{\partial t}+\frac{\partial}{\partial x}\left(\frac{\partial R\left(u_{1}, u_{2}\right)}{\partial u_{\alpha}}\right)=\mu \frac{\partial^{2} u_{\alpha}}{\partial x^{2}}-\mathscr{D} \int_{x_{0}}^{x} \frac{\partial^{2} u_{\alpha}}{\partial y^{2}} d x, \quad \alpha=1,2 .
$$

Уравнения (2.4) отличаются от уравнений (2.1) только вторым слагаемым в правой части уравнений (2.4), учитывающим слабую зависимость решения от переменной $y$. Это слагаемое имеет тот же вид, что и соответствующие члены в уравнениях Кадомцева-Петвиашвили [43] и Хохлова-Заболотской [44]. Коэффициент $\mathscr{D}$ равен половине характеристической скорости изучаемых волн в линейной изотропной среде. Функция $R\left(u_{1}, u_{2}\right)$ имеет вид (1.4).

При расчетах в качестве $x_{0}$ (нижнего предела в интеграле) выбиралось такое значение $x$ впереди изучаемой системы волн, где можно считать, что $u_{1}$ и $u_{2}$ 
принимают невозмущенные постоянные значения. Ниже приводятся результаты численного решения ряда начально-краевых задач для уравнений (2.4), для которых описанные в п. 1.3 автомодельные решения могут представлять асимптотики при $t \rightarrow \infty$.

Уравнения (2.4) позволяют рассматривать поведение решений в системе координат, движущейся в положительном направлении оси $x$ относительно исходной системы координат с некоторой постоянной скоростью. Это приводит к изменению величины $f$ - коэффициента в выражении (1.4), представляющего скорость характеристик в линейном изотропном приближении относительно выбранной системы координат. В дальнейшем величина $f$ будет подбираться исходя из удобства расчетов. При этом линейные и нелинейные члены в левой части системы (2.4) будут считаться одного порядка. Коэффициентам $g, \varkappa, \mu$ и $\mathscr{D}$ можно придать любые значения, если подходящим образом распорядиться единицами измерения $u_{\alpha}, x, t$ и $y$.

Оценим порядок величины члена, ответственного за неодномерность, исходя из предположения, что вязкий и нелинейный члены имеют один и тот же порядок величины, как это имеет место в одномерном случае. Тогда получим, что характерный масштаб изменения величин по оси $x$ равен $L_{x} \sim \mu /\left(\varkappa u^{2}\right)$, а порядок величины нелинейного члена равен $\varkappa u^{3} / L_{x}$. Отношение "пространственного" члена к нелинейному члену в уравнениях (2.4) по порядку величины равно $M=\left(\mathscr{D} / \varkappa u^{2}\right)\left(L_{x} / L_{y}\right)^{2}=\mathscr{D} \mu^{2} /\left(L_{y}^{2} \varkappa^{3} u^{6}\right)\left(L_{x}\right.$ и $L_{y}$ - характерные размеры соответственно по переменным $x$ и $y)$. Ввиду того, что $\mathscr{D} / \varkappa u^{2} \gg 1$, $L_{x} / L_{y} \gg 1$, параметр $M$ может быть как больше, так и меньше единицы. Безразмерный параметр $M$ характеризует интенсивность взаимодействия по переменной $y$ различных участков волны между собой.

Ниже рассматриваются задачи, в которых $u \sim 1$, а полученная оценка позволяет сравнивать между собой результаты различных вариантов расчета. В частности, как видно из выражения для $M$, увеличение $\mathscr{D}$ при сохранении $M$ эквивалентно увеличению масштаба $L_{y}$, характеризующего зависимость решения от $y$.

Ниже приводятся результаты численного решения ряда начально-краевых задач для уравнений (2.4), для которых описанные в п. 1.3 автомодельные решения могут представлять асимптотики при $t \rightarrow \infty$.

Граничные условия $(t \geqslant 0)$ задаются в виде

$$
\begin{array}{rll}
x=l, \quad y>0: & u_{\alpha}=U_{\alpha} \quad(\text { точка } A) ; \\
x=0, \quad y>0: & u_{1}=u_{\alpha}^{*} \quad(\text { точка } B) ; \\
y=0, \quad y=L, \quad 0<x<l: & \frac{\partial u_{\alpha}}{\partial y}=0 .
\end{array}
$$

Начальные условия $(t=0,0<x<l)$ задаются в виде

$$
\begin{array}{ll}
0<y \leqslant y_{1}: & u_{\alpha}=u_{\alpha}^{(2)}(x), \\
y_{1}<y<L: & u_{\alpha}=u_{\alpha}^{(1)}(x) .
\end{array}
$$

В связи с постановкой граничных условий отметим, что задание граничных условий при $y=0$ и $y=L$ в виде равенства нулю производных по $y$ обеспечивает возможность периодического (с периодом $2 L$ ) продолжения решения 
по $y$. Граничные условия при $x=l$ соответствуют состоянию перед волной, которая является быстрой, так что состояние впереди на достаточном удалении от волны можно считать невозмущенным.

При выборе начальных данных (функций $u_{\alpha}^{(1)}(x)$ и $\left.u_{\alpha}^{(2)}(x)\right)$ использовались результаты работы [40] (изложенные в предыдущем пункте), в которой в одномерной постановке рассматривалась задача о взаимодействии метастабильной ударной волны с неоднородностью фона, задаваемого изменением коэффициента $g$ (анизотропии) в течение некоторого промежутка времени $\tau$.

Зависимость функций $u_{\alpha}^{(1)}(x)$ и $u_{\alpha}^{(2)}(x)$ от $x$ в равенствах (2.6) выбиралась следующим образом. В качестве начальных данных $u_{\alpha}^{(1)}(x)$ задавалась стационарная структура метастабильной ударной волны (точка $A$ имела координаты $U_{1}=1, U_{2}=1$, точка $B$ - координаты $u_{1}^{*}=-1.05, u_{2}^{*}=-0.455 ; g=g_{1}=0.03$; $\varkappa=0.5 ; \mu=0.024$ (сплошные линии на рис. 2.9$)$ ). В качестве $u_{\alpha}^{(2)}(x)$ (пунктирные линии на рис. 2.9) выбирались функции, соответствующие некоторому необратимому этапу $t=\tau$ одномерного распада (описанного в п. 2.4) той же стационарной метастабильной ударной волны в результате увеличения $g$ от значения $g=g_{1}=0.03$ до значения $g=g_{2}=0.1$ при прежних значениях остальных параметров (рис. 2.11).

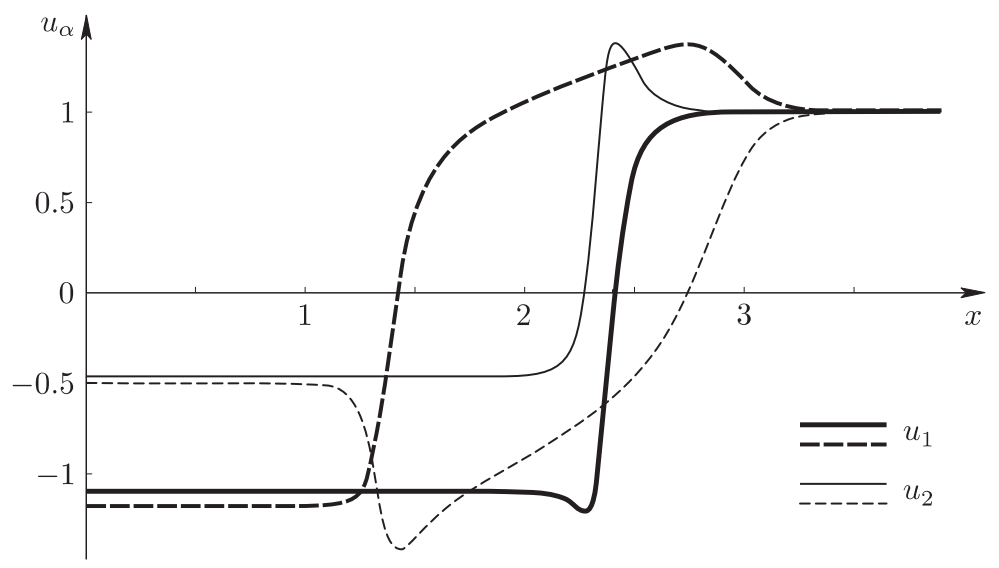

Рис. 2.11. Начальные данные

Начальные данные (2.6) заданы таким образом, что при переходе $y$ через значение $y=y_{1}$ происходит смена начальных условий. При такой постановке начальных условий вследствие существенного различия функций $u_{\alpha}^{(1)}(x)$ и $u_{\alpha}^{(2)}(x)$ разностные уравнения плохо аппроксимируют интегро-дифференциальные уравнения (2.4). Для устранения этого недостатка в начальных условиях были введены "переходные зоны" по переменной $у$ так, что при изменении $у$ имела место не скачкообразная, а постепенная смена типа начальных условий [41]. 
Начальные данные на интервале $y_{1} \leqslant y \leqslant y_{1}+y_{p}$ ( $y_{p}-$ ширина переходной зоны) задавались следующим образом:

$$
\begin{gathered}
u_{\alpha}^{(p)}(x)=(1-f(s)) u_{\alpha}^{(1)}(x)+f(s) u_{\alpha}^{(2)}(x), \\
s=\frac{y-y_{1}}{y_{p}}, \quad f(s)=s^{2}\left(2-s^{2}\right) .
\end{gathered}
$$

Функция $f(s)$ осуществляет сглаживание. Она удовлетворяет условиям

$$
f(0)=0, \quad f(1)=1, \quad f^{\prime}(0)=0, \quad f^{\prime}(1)=0 .
$$

На рис. 2.11 значения $u_{\alpha}^{(2)}(x)$ слева от области быстрого изменения этих функций немного отличаются от значений $u_{\alpha}^{(1)}(x)$ в той же области. Это отличие вызвано тем, что в процессе распада метастабильной ударной волны, приведшего к функциям $u_{\alpha}^{(2)}(x)$, налево ушла медленная ударная волна, которая слегка изменила значения $u_{\alpha}$ и вышла за пределы расчетной области. В пределах расчетной области располагаются только быстрые волны, слегка искаженные вязкостью. До проведения расчетов было специально проверено, что приход на левую границу $(x=0)$ медленной волны не приводит к появлению сколько-нибудь заметной отраженный быстрой волны (которая могла бы исказить изучаемый процесс).

С учетом наличия переходных зон начальные условия (2.6) были записаны следующим образом:

$$
\begin{aligned}
0 \leqslant y \leqslant y_{1}: & u_{\alpha}=u_{\alpha}^{(2)}(x) ; \\
y_{1} \leqslant y \leqslant y_{1}+y_{p}: & u_{\alpha}=u_{\alpha}^{(p)}(x) ; \\
y_{1}+y_{p} \leqslant y \leqslant L_{y}: & u_{\alpha}=u_{\alpha}^{(1)}(x) .
\end{aligned}
$$

Расчеты начально-краевой задачи $(2.4),(2.5),(2.7)$ позволили пронаблюдать процессы восстановления и распада структуры метастабильной ударной волны. Последовательно опишем и продемонстрируем этот процесс на графиках.

В одном из вариантов расчета начальные условия $(t=0)$ были взяты в следующем виде: в двух последовательных слоях $\left(y_{j}=\right.$ const, $y_{j+1}=y_{j}+\Delta y$, $j=1,2)$ задаются начальные условия $(2.7 \mathrm{a})$, затем 10 последовательных слоев $(j=3, \ldots, 12)$ соответствуют переходной зоне с начальными условиями $(2.7 \mathrm{~b})$ и два слоя $(j=13,14)$ соответствуют начальным условиям $(2.7 \mathrm{c})$ (невозмущенная метастабильная ударная волна). На рис. 2.12 представлен график функции $u_{1}(x, y)$ при $t=0$. Поставленная начально-краевая задача решалась при различных значениях параметра $\mathscr{D}$.

При $\mathscr{D}=0, \Delta y=2$ (в системе уравнений (2.4) отсутствуют члены, описывающие взаимодействие между слоями по переменной $y$ ) с увеличением расчетного промежутка времени $t$ происходят следующие изменения. На пяти слоях промежуточной области, примыкающих к границе, представляющей решение второго типа, постепенно формируется асимптотика решения второго типа. На остальных слоях промежуточной области формируется асимптотика решения первого типа.

При малых значениях параметра $\mathscr{D}(\mathscr{D}=0.5,1,2,3$ и, соответстсвенно, $M=$ $\left.3.6735 \cdot 10^{-7}, 7.347 \cdot 10^{-7}, 1.4694 \cdot 10^{-6}, 2.2041 \cdot 10^{-6}\right), \Delta y=2$ с увеличением 


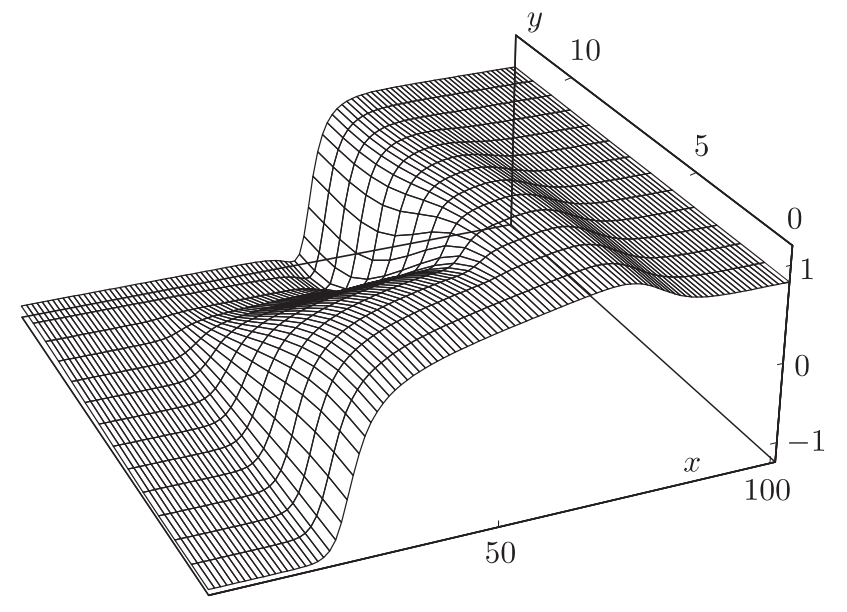

Рис. 2.12. Начальные данные, включающие "переходные зоны"

расчетного промежутка времени постепенно формируется асимптотика первого типа на переходном слое $(j=12)$, граничащем со слоем, на котором задана асимптотика решения первого типа $(j=13)$. Затем асимптотика первого типа формируется на слое $j=11$ и т. д. Перестройка решения на каждом из последующих слоев сопровождается излучением медленной волны, распространяющейся налево. Эта медленная волна при взаимодействии с границей не дает отраженных возмущений. Таким образом, во всей расчетной зоне формируется асимптотика решения первого типа.

Эволюцию решения поставленной начально-краевой задачи при $\mathscr{D}=30$ $\left(M=2.2041 \cdot 10^{-5}\right), \Delta y=2$ можно наблюдать на рис. $2.13 a-d(t=0.18$, $0.6,1.8,2.7)$, где изображены графики функции $u_{1}(x, y)$ в последовательные моменты времени. На рис. 2.13 а видно начало формирования асимптотики решения второго типа на слоях $j=8,9,10$. На рис. $2.13 b$ асимптотика решения второго типа формируется на слоях $j=7, \ldots, 14$. На рис. $2.13 c$, $d$ виден процесс завершения формирования асимптотики решения второго типа. Таким образом, при $\mathscr{D}=30$ и наличии одинакового количества слоев, представляющих асимптотику решения первого типа, и слоев с асимптотикой второго типа формируется асимптотика решения второго типа, т.е. исходная метастабильная ударная волна перестает существовать. Если изменить постановку начально-краевой задачи и в начальный момент времени задать число слоев с асимптотикой первого типа, существенно бо́льшее числа слоев с асимптотикой второго типа, и при этом не изменять остальные параметры задачи, то на всех слоях сформируется асимптотика первого типа, т. е. изучаемая метастабильная ударная волна восстановится.

На рис. $2.14 a-d$ представлена эволюция решения начально-краевой задачи, имеющей существенно больший характерный размер $L$, чем начально-краевая задача на рис. 2.13, и существенно больший параметр $\mathscr{D}=100(M=$ $\left.6.6241 \cdot 10^{-5}\right)$. При $t=0$ в четырех последовательных слоях $\left(y_{j}=\right.$ const, $\left.y_{j+1}=y_{j}+\Delta y, \quad j=1, \ldots, 4\right)$ задаются начальные условия $(2.7 \mathrm{a})$, затем 10 

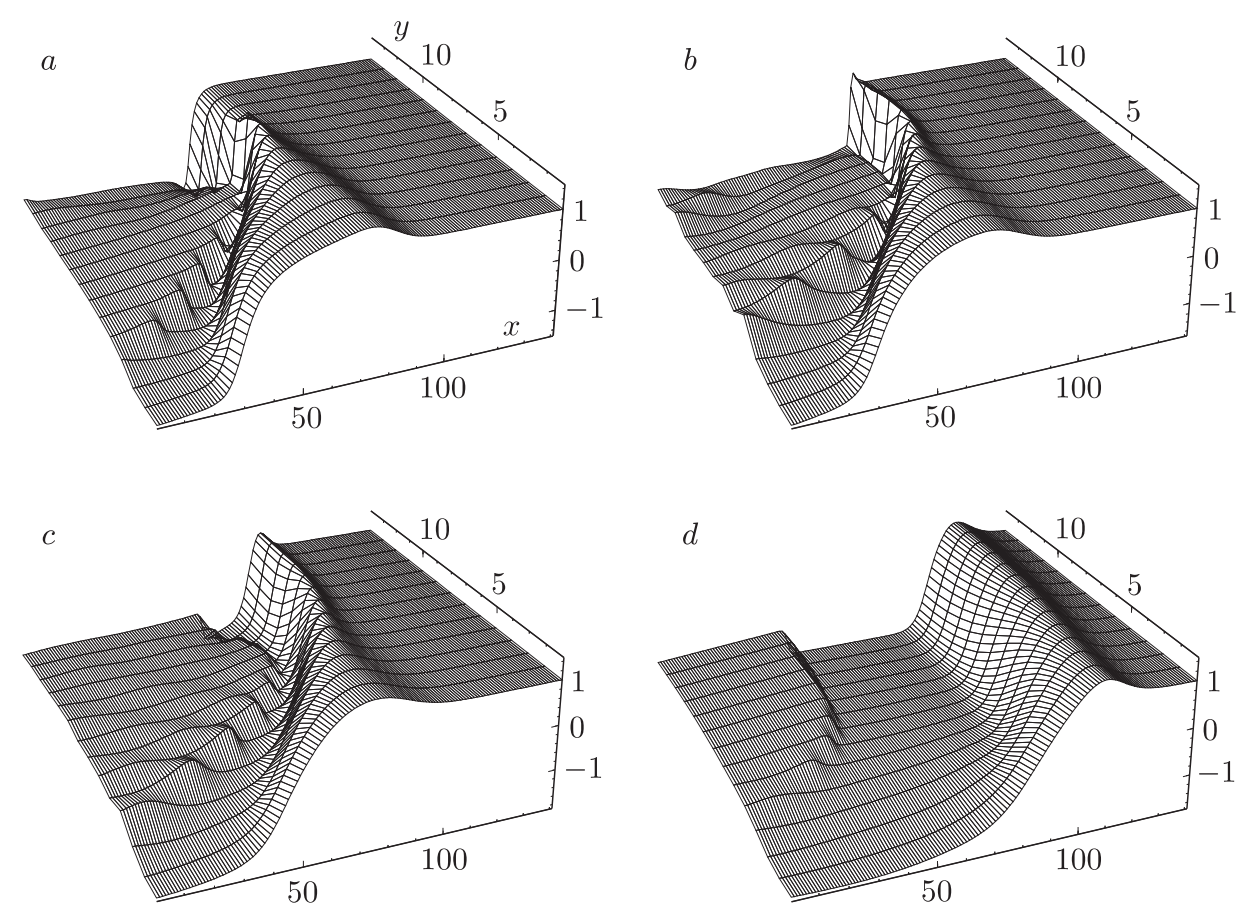

Рис. 2.13. Перестройка решения

последовательных слоев $(j=5, \ldots, 14)$ соответствуют переходной зоне с начальными условиями $(2.7 \mathrm{~b})$ и двадцать один слой $(j=15, \ldots, 35)$ соответствует начальным условиям (2.7c) (невозмущенная метастабильная ударная волна), $\Delta y=2$. На рис. $2.14 a-c(t=0.6,1.2,2.4)$ можно наблюдать процесс формирования асимптотики первого типа (метастабильная ударная волна, функция $\left.u_{1}(x, y)\right)$. В последующий момент времени $t=3.63$ (рис. $2.14 d$ ) видно, что метастабильная ударная волна сформировалась при всех значениях $y$.

Была решена эта же начально-краевая задача при $\mathscr{D}=200,1000(M=$ $\left.2.35 \cdot 10^{-5}, 1.175 \cdot 10^{-4}\right)$. При этих значениях параметра $\mathscr{D}$ метастабильная ударная волна с течением времени восстановилась при всех значениях $y$. Этот пример и другие расчеты показали, что решение задачи, когда ширина области с невозмущенной метастабильной ударной волной достаточно велика, всегда приводит к восстановлению метастабильной ударной волны при любом значении параметра $\mathscr{D}$.

Аналогичные начально-краевые задачи рассчитывалась при разных значениях параметров анизотропии $\left(g_{1}, g_{2}\right)$, разной величине отрезка, характеризующего размер неоднородности $y_{p}$, разных положениях начальной точки $A$ и разных положениях точки $B$, описывающей левое граничное условие.

Результаты расчетов приводят к выводу, что возмущения, периодические по переменной вдоль фронта метастабильной ударной волны (даже если это возмущения конечной амплитуды), не приводят к распаду метастабильной удар- 

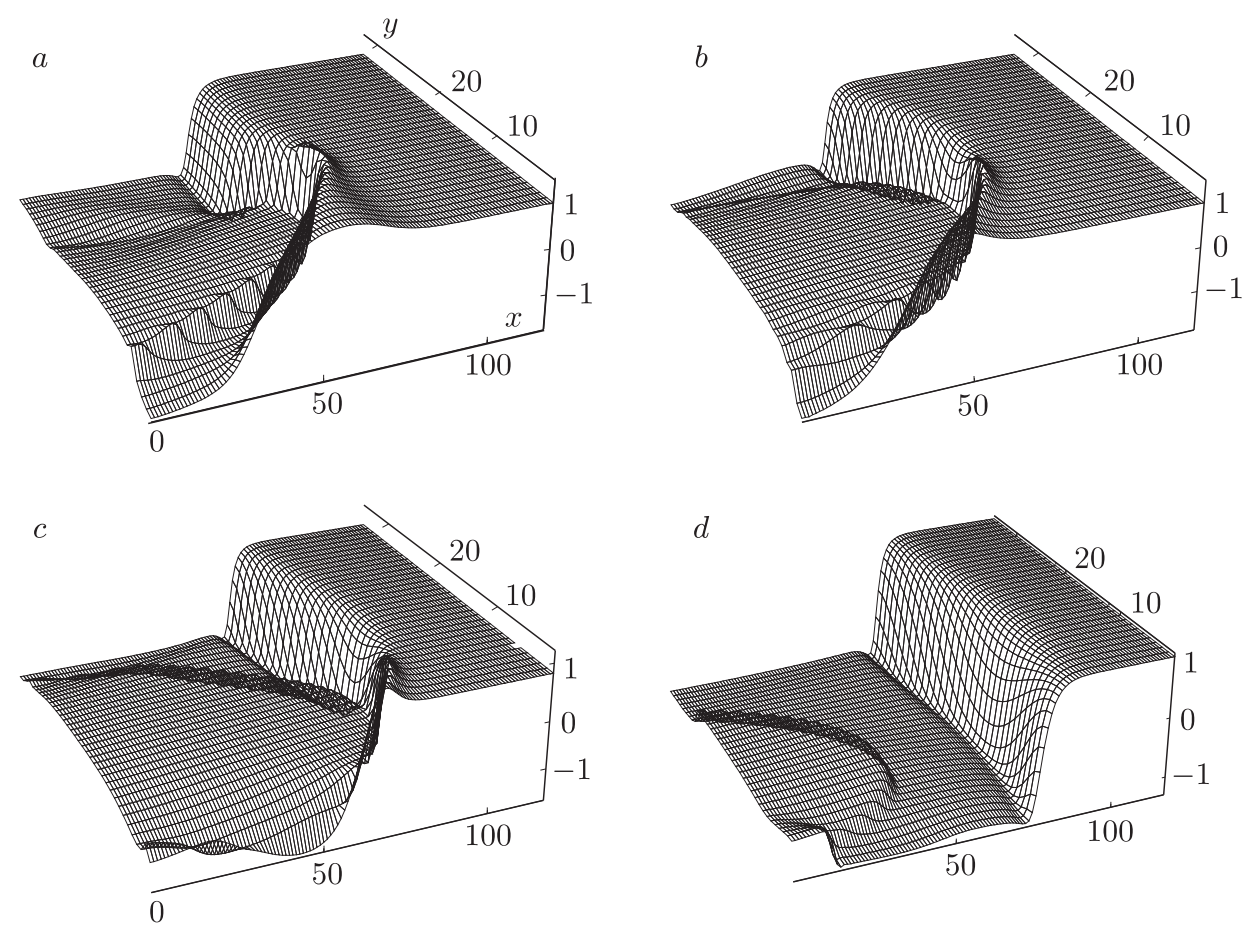

Рис. 2.14. Перестройка решения

ной волны если масштаб $L_{y}$, характеризующий период возмущений вдоль этой волны, велик (или параметр $M$ - мал). При взаимодействии метастабильной ударной волны с изолированными возмущениями можно также ожидать ее восстановления. В случае малости $L_{y}(M-$ большое число) при определенных условиях может произойти распад метастабильной ударной волны, если на периоде возмущений по переменной у метастабильная волна занимает относительно малую часть.

2.6. Выводы. Таким образом, полученные результаты говорят о большой устойчивости исследуемой метастабильной ударной волны, о возможности “залечивания ею ран", нанесенных сильными, но ограниченными в пространстве внешними воздействиями. Это позволяет предположить, что метастабильные ударные волны могут реально существовать. В этом параграфе продемонстрировано, что каждое из двух решений, полученных в 11 как решение задачи о поршне в “гиперболической" постановке, может представлять асимптотику при больших временах решения вязко-упругой задачи при подходящем изменении действия поршня в течение начального промежутка времени. При этом в каком-то (не вполне определенном) смысле в качестве асимптотики чаще образуется более простое решение, содержащее метастабильную ударную волну (названное решением первого типа). В частности, при распаде произвольного разрыва, образовавшегося в результате взаимодействия двух ударных волн, всегда возникает решение первого типа. Структура этой волны проявляет 
устойчивость по отношению к одномерным и двумерным возмущениям конечной амплитуды.

\section{§ 3. Асимптотическое поведение нелинейных волн в упругих средах с дисперсией и диссипацией}

В этом параграфе, следуя работам [45]-[48], мы рассматриваем нелинейные волны в среде, описываемой системой уравнений, в которой члены низшего порядка дифференцирования образуют, как и в предыдущем параграфе, гиперболическую систему нелинейной теории упругости. Члены с производными высшего порядка помимо вязкости, которая учитывалась в предыдущем параграфе, описывают также дисперсию. Присутствие дисперсионного члена, если он достаточно велик по сравнению с вязким членом, как будет видно из дальнейшего, приводит к изменению множества допустимых разрывов, которое определяется как множество разрывов, имеющих стационарную структуру. Это множество приобретает сложное строение, что приводит к изменению решений задач, содержащих допустимые разрывы. Появляется множество неклассических, особых разрывов с дополнительными соотношениями на них. Часть классических разрывов (ударных волн) оказываются недопустимыми. Решения автомодельных задач оказываются неединственными, причем число возможных решений неограниченно растет с ростом относительного влияния дисперсии по отношению к вязкости. Численное решение уравнений в частных производных с учетом дисперсионных и вязких членов позволяет выделять единственное решение в задачах с автомодельной асимптотикой. Формирование той или иной асимптотики зависит от деталей постановки задачи, отсутствующих в автомодельной постановке.

Далее изучаются решения системы уравнений, описывающей поведение нелинейных волн в одной из моделей упругих композитных материалов, т. е. в упругой среде, обладающей внутренней структурой, связанной с периодичностью свойств среды:

$$
\left\{\begin{array}{c}
\frac{\partial u_{1}}{\partial t}+\frac{\partial}{\partial x}\left(\frac{\partial R\left(u_{1}, u_{2}\right)}{\partial u_{1}}\right)=\mu \frac{\partial^{2} u_{1}}{\partial x^{2}}+m \frac{\partial^{2} u_{2}}{\partial x^{2}}, \\
\frac{\partial u_{2}}{\partial t}+\frac{\partial}{\partial x}\left(\frac{\partial R\left(u_{1}, u_{2}\right)}{\partial u_{2}}\right)=-m \frac{\partial^{2} u_{1}}{\partial x^{2}}+\mu \frac{\partial^{2} u_{2}}{\partial x^{2}} \\
\mu, m=\text { const, } \quad \mu \geqslant 0 .
\end{array}\right.
$$

В дальнейшем будет предполагаться, что $m / \mu \gg 1$.

Система (3.1) отличается от (2.1) тем, что учитывает дисперсионные эффекты, которые существенны при описании структуры разрывов. Члены, описывающие дисперсию, содержат множитель $m$. Члены, содержащие $\mu$, обуславливают диссипацию. При написании системы (3.1) предположено, что при малой нелинейности члены, описывающие дисперсию и диссипацию, сохраняют тот же вид, что и в линейных волнах. Вид дисперсионных членов в (3.1) соответствует одному из вариантов строения композитов, поведение которых исследовано в [49]. 
Множители $m$ и $\mu$ стоят при старших производных в системе (3.1), поэтому при изучении крупномасштабных явлений эта система будет совпадать с системой (1.3). Вид функции $R\left(u_{1}, u_{2}\right)$ предполагается таким же, как и в системе (1.3) (см. (1.4)). Соотношения на разрывах системы уравнений (1.3), следующие из законов сохранения, и вид ударной адиабаты описаны в 1 .

3.1. Структура разрывов. Для системы уравнений (3.1) разыскиваются решения вида $u_{\alpha}=u_{\alpha}(\xi), \xi=-x+W t, W=$ const - скорость разрыва. Предполагается, что при $\xi \rightarrow-\infty$ величины $u_{\alpha}$ стремятся к значениям $U_{\alpha}$, соответствующим состоянию перед разрывом, а при $\xi \rightarrow+\infty$ они принимают значения $u_{\alpha}$, соответствующие состоянию за разрывом, т.е. состоянию, принадлежащему некоторой точке ударной адиабаты. При $\mu \rightarrow 0, m \rightarrow 0$ и $m / \mu=$ const эти решения переходят в разрывы, изменение величин в которых совпадает с изменением величин в соответствующем квазипоперечном разрыве.

Для функций $u_{\alpha}(\xi)$ имеем систему двух обыкновенных дифференциальных уравнений второго порядка. Один раз проинтегрировав эти уравнения по $\xi$, получим

$$
\begin{gathered}
\left\{\begin{array}{l}
\mu \frac{d u_{1}}{d \xi}+m \frac{d u_{2}}{d \xi}=-\frac{\partial Z}{\partial u_{1}}, \\
-m \frac{d u_{1}}{d \xi}+\mu \frac{d u_{2}}{d \xi}=-\frac{\partial Z}{\partial u_{2}}
\end{array}\right. \\
Z\left(u_{1}, u_{2}\right)=R\left(u_{1}, u_{2}\right)-\frac{1}{2} W\left(u_{1}^{2}+u_{2}^{2}\right)-Q_{1} u_{1}-Q_{2} u_{2}, \\
Q_{\alpha}=U_{\alpha}\left(f-W+(-1)^{\alpha} g-\varkappa\left(U_{1}^{2}+U_{2}^{2}\right)\right) .
\end{gathered}
$$

Заметим, что

$$
\frac{d Z}{d \xi}=-\mu\left[\left(\frac{d u_{1}}{d \xi}\right)^{2}+\left(\frac{d u_{2}}{d \xi}\right)^{2}\right]<0 .
$$

Если изменять значения констант $\mu$ и $m$, сохраняя значение их отношения, то с точностью до масштаба по переменной $\xi$ решения уравнений (3.2) не изменяются.

Система (3.2) с точностью до обозначений совпадает с системой, описывающей структуру нелинейных электромагнитных волн в слабоанизотропных магнетиках [22], [46]. В [45], [46] при изучении нелинейных волновых процессов, когда при описании структуры разрывов существенны процессы дисперсии и диссипации, было выявлено существенное влияние дисперсии на множество допустимых разрывов.

Координаты особых точек системы (3.2) определяются системой уравнений

$$
\frac{\partial Z}{\partial u_{\alpha}}=0, \quad \alpha=1,2 .
$$

Координаты этих точек могут соответствовать значениям $u_{\alpha}(\xi)$ при $\xi= \pm \infty$. Структуре ударной волны соответствует интегральная кривая, соединяющая две особые точки. Подчеркнем, что в рассматриваемом случае, когда $m / \mu \gg 1$, интегральные кривые системы (3.2) близки к линиям уровня $Z\left(u_{1}, u_{2}\right)=\mathrm{const}$ 
и составляют с ними малый угол. Поведение интегральных кривых для сред с $\varkappa>0$ и сред с $\varkappa<0$ существенно различается.

Рассмотрим сначала случай $\varkappa>0$. На рис. 3.1 а представлены линии уровня функции $Z\left(u_{1}, u_{2}\right)$ для значений параметров $f=1, g=1, \varkappa=4, W=-16$ и состояния перед разрывом $U_{1}=2, U_{2}=0.4$ (точка $A$ ). На рис. $3.1 b$ представлена диаграмма эволюционности, на которой отмечены стационарные точки при выбранном значении $W$. Буквами $O, C, B, G$ отмечены одни и те же точки на обоих рисунках $3.1 a, b$. Поверхность, представляемая графиком функции $Z\left(u_{1}, u_{2}\right)$, напоминает кратер вулкана - гора с понижением посередине (точка $O$ - минимум), двумя максимумами $B$ и $G$ и перевалами $A$ и $C$ на краю кратера.
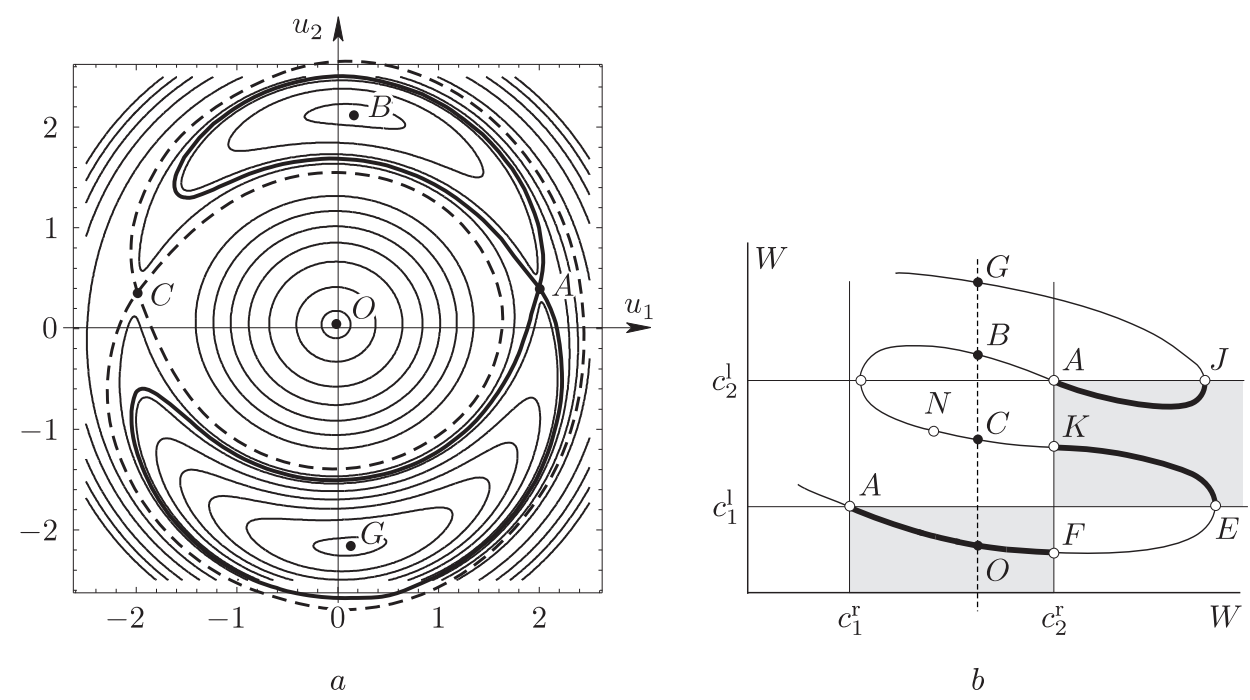

Рис. 3.1. Линии уровня $(\varkappa>0)$

Наибольший интерес представляет интервал изменения скорости $W_{N}<W<$ $\min \left(c_{2}^{\mathrm{r}}, W_{E}\right)\left(W_{N}, W_{E}-\right.$ скорости разрывов $A \rightarrow N$ и $A \rightarrow E$ соответственно, см. рис. $1.2 b, d)$, в котором существует пять особых точек у системы (3.2): начальная точка $A$ - седло, устойчивый фокус $O$, седло $C$ и два неустойчивых фокуса $B$ и $G$. При $W>c_{2}^{\mathrm{r}}$ и $W<W_{N}$ особых разрывов нет, так как в этом случае особая точка $A$ на плоскости $\left(u_{1}, u_{2}\right)$ представляет фокус с выходящими интегральными кривыми. В этом случае невозможно существование особых разрывов, структура которых, как будет видно из дальнейшего, представляется интегральной кривой, соединяющей два седла.

Каждая из двух интегральных кривых, вышедших из точки $A$, при $\xi \rightarrow+\infty$ должна прийти в одну из особых точек $O, C$ или уйти в бесконечность. Примеры двух характерных стационарных структур приведены на рис. $3.2 a, b$. Приход интегральной кривой в седловую точку $C$ следует рассматривать как исключительный случай, который возможен при специальном дискретном наборе значений скорости $W=W_{i}^{*}$. 


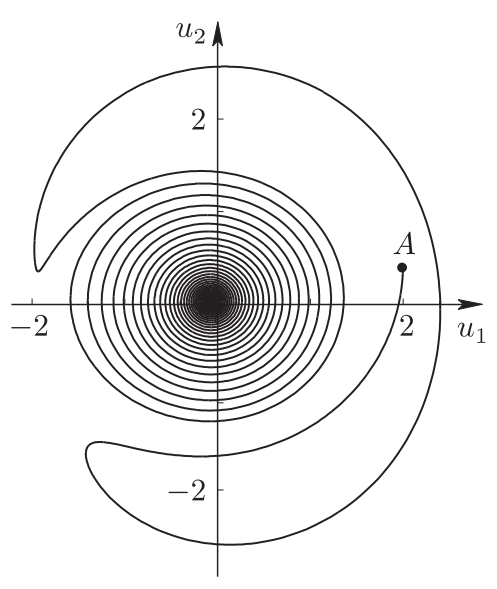

$W=-16.066$

a

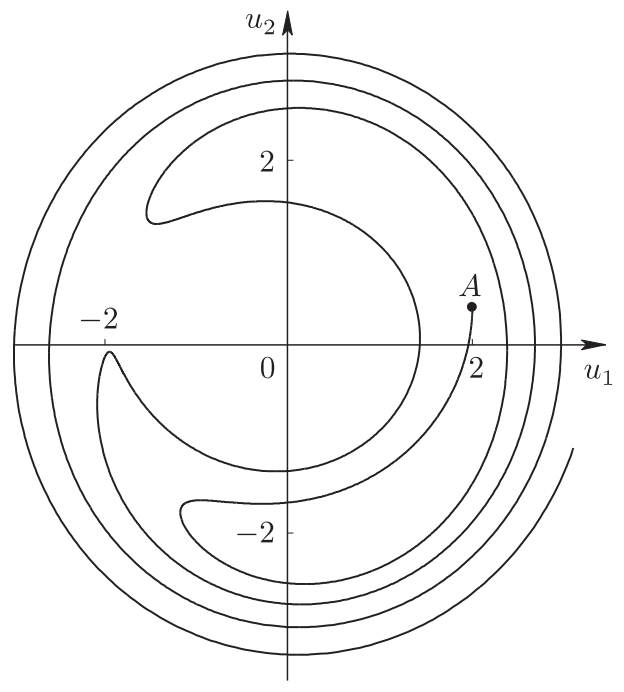

$W=-15.94$

b

Рис. 3.2. Стационарные структуры разрывов $(\varkappa>0)$

Интегральные кривые, вышедшие из точки $A$, движутся в сторону уменьшения функции $Z$, пересекая линии $Z=$ const под малыми (порядка $\mu / m$ ) углами. Прежде чем любая из двух интегральных кривых пересечет пунктирную линию $Z\left(u_{1}, u_{2}\right)=Z(C)$ (рис. 3.1), она при достаточно малых $\mu / m$ сделает много оборотов вокруг жирной сплошной линии $\left(Z\left(u_{1}, u_{2}\right)=Z(A)\right)$. При изменении $W$ точка пересечения интегральной кривой и пунктирной линии движется по пунктирной кривой. Когда упомянутое число оборотов меняется на единицу, точка пересечения обегает всю пунктирную линию, дважды побывав в точке $C$. При соответствующих выделенных значениях $W=W^{*}$ существует структура разрыва $A \rightarrow C$. Этот разрыв является особым, поскольку существование его структуры накладывает ограничение на значение его скорости, что представляет дополнительное условие, не следующее из законов сохранения. Если точка пересечения интегральной кривой с пунктирной линией находится на внутренней части пунктирной линии (окружающей точку $O$ ), то эта интегральная кривая далее приходит в точку $O$, представляя структуру медленной ударной волны $A \rightarrow O$. При переходе точки пересечения интегральной кривой, вышедшей из точки $A$, и пунктирной линии уровня с внешней части этой линии уровня (проходящей через точку $C$ и окружающей точки $B$ и $C$ ) на внутреннюю (окружающую только точку $O$ ) рассматриваемая интегральная кривая приходит в точку $C$ и описывает структуру особого разрыва $A \rightarrow C$ (это возможно для выделенных значений $W$ ). Если интегральная кривая пересекает внешнюю часть пунктирной кривой, то она уходит в бесконечность и не соответствует структуре какого-либо разрыва.

На рис. 3.3, 3.4 приведены примеры стационарных структур особых разрывов, полученные численно для значений параметров диссипации $\mu=0.05$, 
дисперсии $m=3(m / \mu=60)$ и прежних значений $U_{1}=2, U_{2}=0.4, f=1$, $g=1, \varkappa=4$. На рис. 3.3 представлены простые структуры особых разрывов, на рис. 3.4 - сложные структуры для больших значений $W=W^{*}$, демонстрирующие различные виды обхода выделенной на рис. 3.1 a "восьмерки".

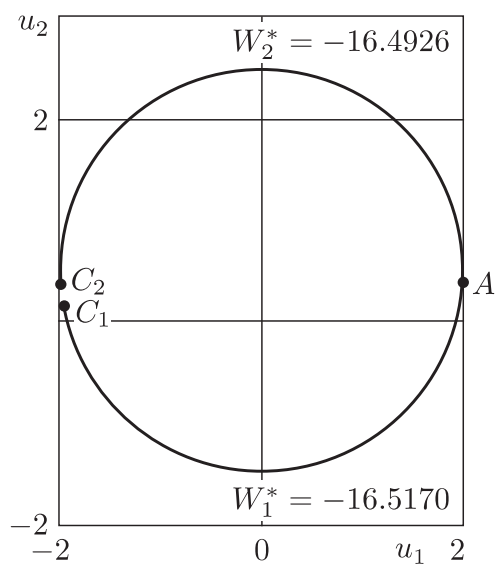

Рис. 3.3. Стационарные структуры особых разрывов $(\varkappa>0)$

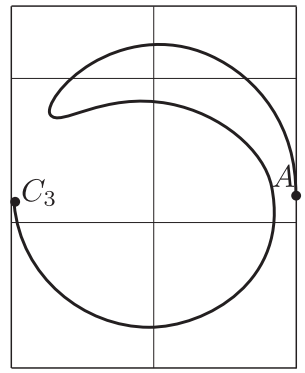

$W_{3}^{*}=-16.3296$

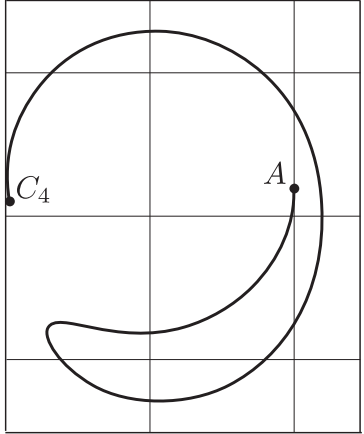

$W_{4}^{*}=-16.0670$

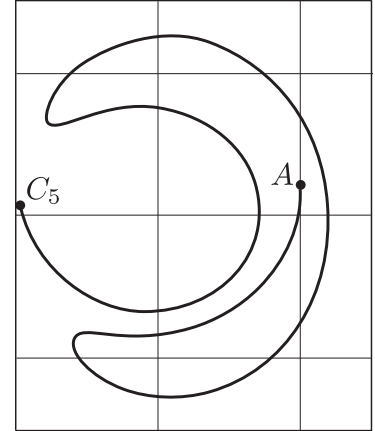

$W_{5}^{*}=-15.9455$

Рис. 3.4. Стационарные структуры особых разрывов $(\varkappa>0)$

При выбранном отношении $m / \mu=60$ в результате численного счета найден набор значений набор $W_{i}^{*}(i=1, \ldots, 5)$, при которых существуют особые разрывы. Точки $C_{i}(i=1, \ldots, 5)$, соответствующие состояниям за особыми разрывами, отмечены на ударной адиабате жирными точками (рис. 3.5). Эти выделенные значения $W_{i}^{*}(i=1, \ldots, 5)$ определяют также концы жирных отрезков (рис. $3.5 b, d$ ), соответствующих допустимым разрывам, на априорно эволюционной части ударной адиабаты. На рис. $3.5 a-d$ представлены фрагменты ударных адиабат и диаграммы эволюционности для тех же параметров $f, g, \varkappa, U_{\alpha}$, что и кривые, изображенные на рис. 1.2 и описанные в п. 1.2. 
Априорно эволюционные отрезки $A F$ (рис. $3.5 a, b)$ и $A E$ (рис. $3.5 c, d$ ) ударных адиабат разбиваются на участки, причем часть из них не отвечает допустимым разрывам. На априорно неэволюционных отрезках $K N$ (рис. $3.5 a, b$ ) и $E N$ (рис. $3.5 c, d$ ) отмечены точки $C_{i}$, отвечающие особым разрывам, которые оказываются эволюционными в силу дополнительного условия $W=W_{i}^{*}$.

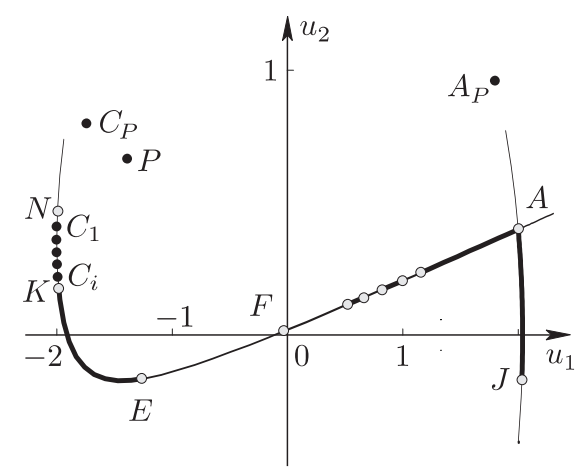

$a$

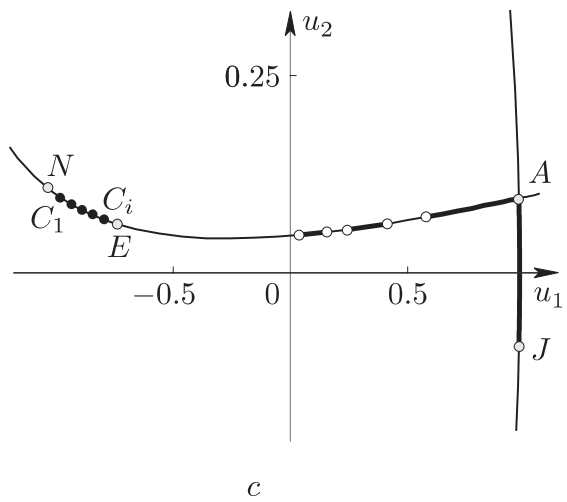

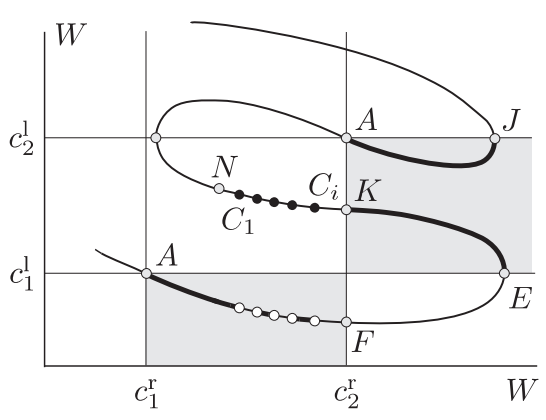

$b$

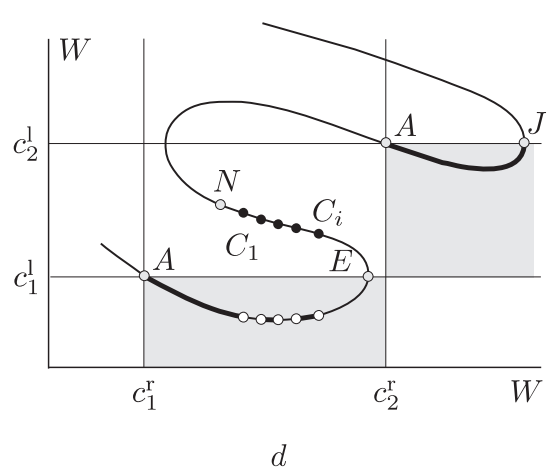

Рис. 3.5. Допустимая часть ударной адиабаты для волн в композитных материалах: жирные кривые и черные точки $(\varkappa>0)$

Рассмотрим теперь поведение интегральных кривых в случае $\varkappa<0$. На рис. 3.6 представлены линии уровня функции $Z\left(u_{1}, u_{2}\right)$ для значений параметров $f=1, g=1, \varkappa=-2, W=4.6$ и состояния перед разрывом $U_{1}=1$, $U_{2}=0.1$ (точка $A$ ). На этом же рисунке представлена диаграмма эволюционности, на которой отмечены стационарные точки при выбранном значении $W$.

Наибольший интерес представляет интервал изменения скорости $W_{E}<$ $W<W_{H}\left(W_{E}, W_{H}-\right.$ скорости разрывов $A \rightarrow E$ и $A \rightarrow H$ соответственно, см. рис. 1.3), в котором существует пять особых точек у системы (3.2): начальная точка $A$ - седло, два устойчивых фокуса $B$ и $G$ (минимумы функции $\left.Z\left(u_{1}, u_{2}\right)\right)$, седло $C$ и неустойчивый фокус $O$ (максимум функции $Z\left(u_{1}, u_{2}\right)$ ). График функции $Z\left(u_{1}, u_{2}\right)$ напоминает круговой желоб переменной глубины. 


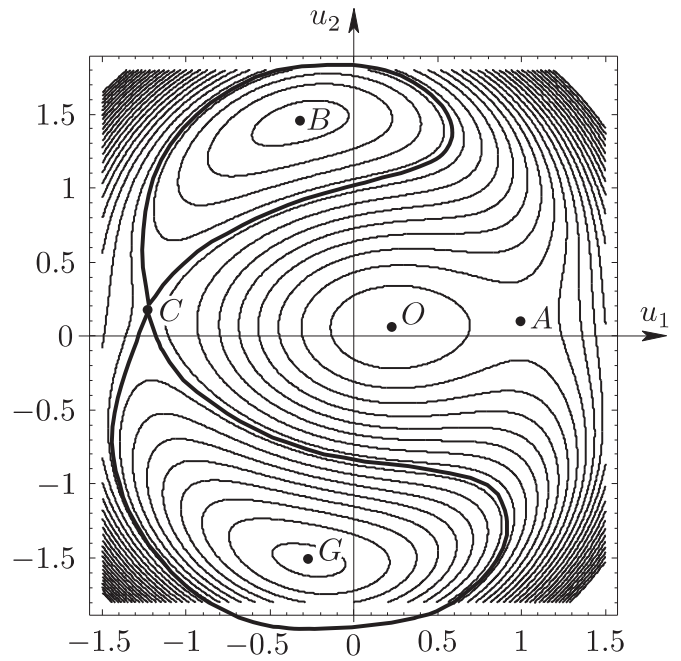

$a$

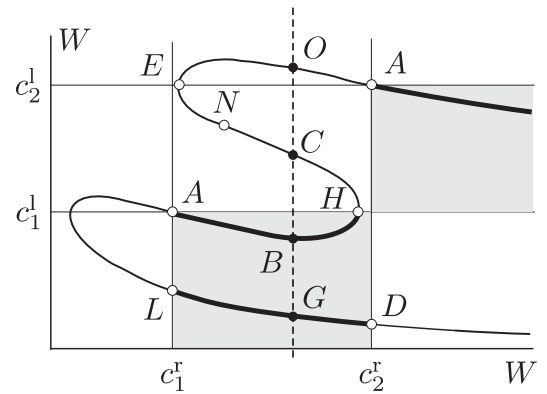

$b$

Рис. 3.6. Линии уровня $(\varkappa<0)$

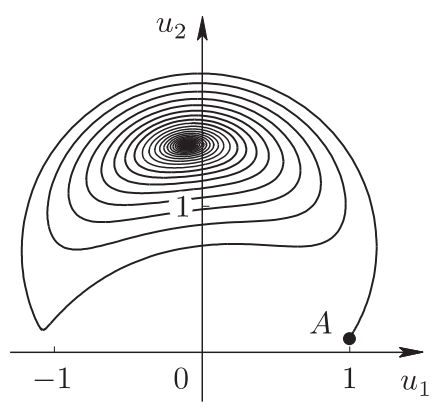

$W=4.199$

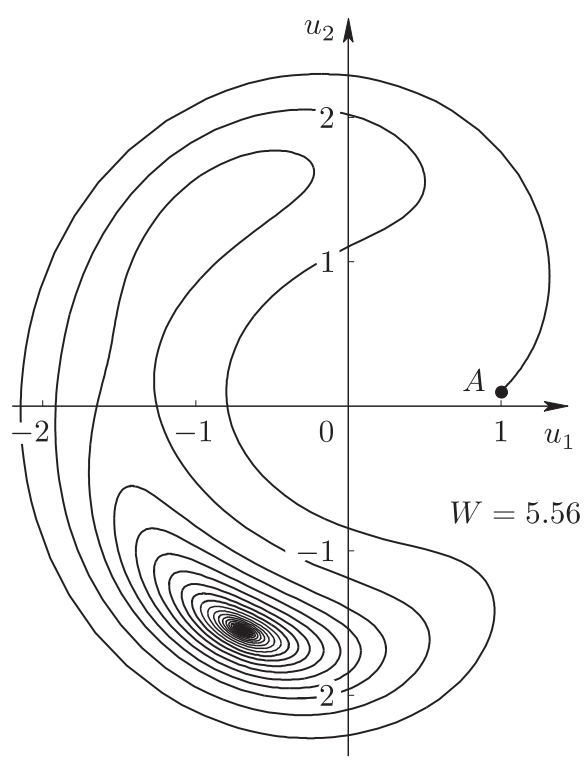

Рис. 3.7. Стационарные структуры разрывов $(\varkappa<0)$

Поскольку функция $Z\left(u_{1}, u_{2}\right)$ растет при достаточном удалении от начала координат, каждая из двух интегральных кривых, вышедших из точки $A$, при $\xi \rightarrow+\infty$ должна прийти в одну из особых точек $B, G, C$. На рис. 3.7 представлены примеры стационарных структур, одна из которых приходит в точку $B(W=4.199)$, вторая - в точку $G(W=5.56)$. Приход интегральной кривой в седловую точку $C$ следует рассматривать как исключительный слу- 
чай, который возможен при специальном дискретном наборе значений скорости $W=W_{i}^{*}$.

Исследование интегральных кривых при $\varkappa<0$ во многом подобно исследованию при $\varkappa>0$. Число оборотов, которые сделает интегральная кривая вокруг выделенной на рис. 3.6 линии уровня в виде жирной “восьмерки”, прежде чем остановится в стационарной точке $C$, зависит от угла наклона интегральной кривой по отношению к линии уровня. Этот угол, согласно предыдущему, имеет порядок величины $\mu / m$. Если разность $Z(A)-Z(C)$ конечна, а величина $\mu / m$ мала, то число оборотов велико. Достаточно малого изменения $W$ (порядка $\mu / m)$, чтобы число оборотов вокруг "восьмерки" изменилось на единицу. При этом точка пересечения интегральной кривой и выделенной восьмерки делает полный оборот по восьмерке, дважды побывав в точке $C$. Значения $W^{*}$, при которых интегральные кривые соединяют точки $A$ и $C$, разделены малыми интервалами порядка $\mu / m$. Каждое значение $W^{*}$ соответствует точке на ударной адиабате. Эта точка лежит на априорно неэволюционной части ударной адиабаты, но поскольку ей соответствует выделенное значение $W^{*}$, которое следует рассматривать как дополнительное соотношение на разрыве, то разрыв $A \rightarrow C$ эволюционный. Разрывы такого типа были названы особыми.

На рис. 3.8, 3.9 приведены примеры стационарных структур особых разрывов, полученные численно для значений параметров диссипации $\mu=0.05$, дисперсии $m=1.5(m / \mu=30)$ и прежних значений $U_{1}=1, U_{2}=0.1, f=1$, $g=1, \varkappa=-2$. На рис. 3.8 представлены простые структуры особых разрывов, на рис. 3.9 - сложные структуры для больших значений $W=W^{*}$, демонстрирующие различные виды обхода выделенной на рис. 3.6 "восьмерки”.

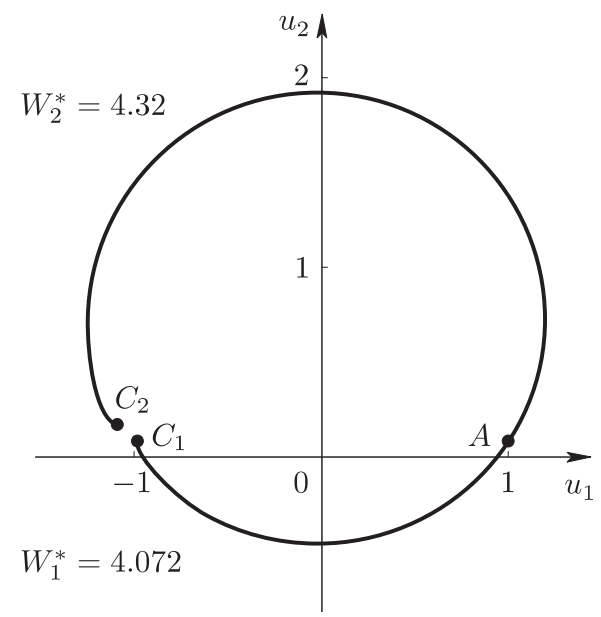

Рис. 3.8. Стационарные структуры особых разрывов $(\varkappa<0)$

При выбранном отношении $m / \mu=30$ в результате численного счета найден набор значений $W_{i}^{*}(i=1, \ldots, 7)$, при которых одна из двух возможных интегральных кривых, вышедших из начальной седловой точки $A$ в противоположных направлениях, приходит в седловую точку $C_{i}(i=1, \ldots, 7)$. Значения $W_{i}(i=1, \ldots, 7)$ соответствуют особым разрывам и одновременно опреде- 


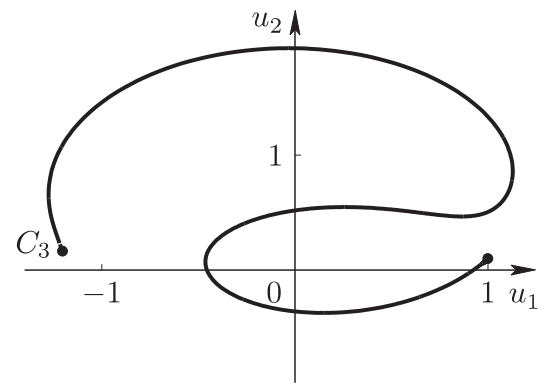

$W=4.386$

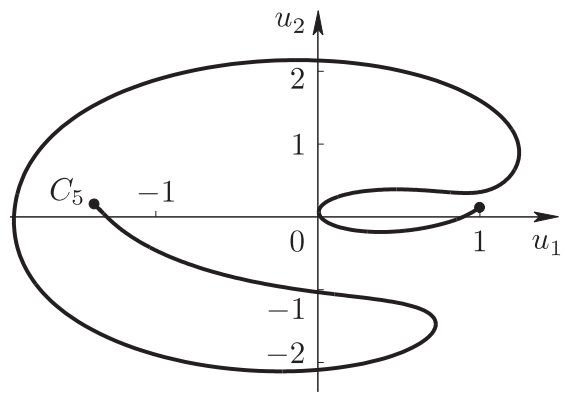

$W=5.12$

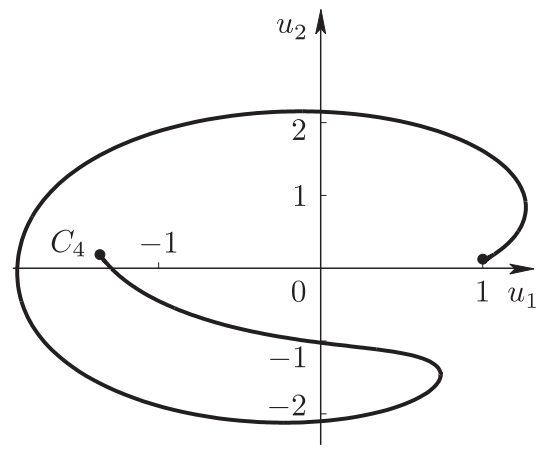

$W=5.1$

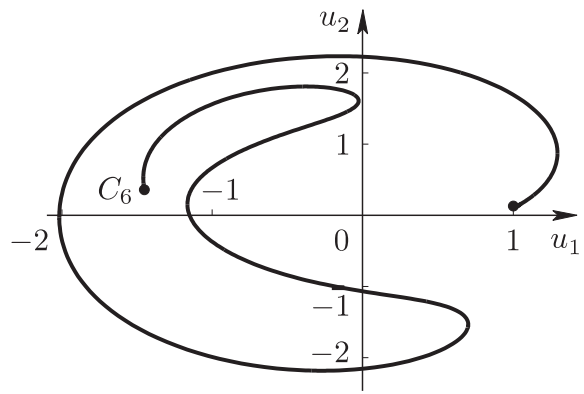

$W=5.41$

Рис. 3.9. Стационарные структуры особых разрывов $(\varkappa<0)$

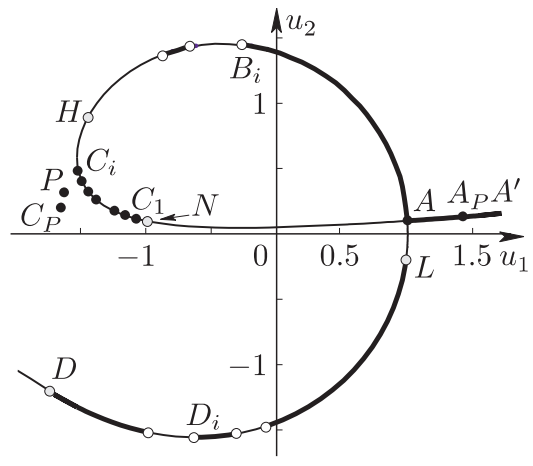

$a$

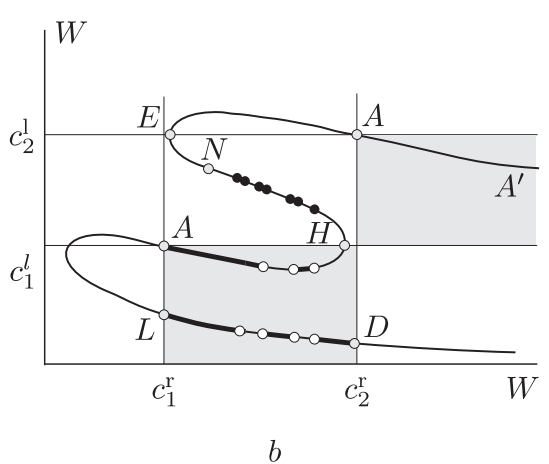

$b$

Рис. 3.10. Допустимая часть ударной адиабаты для волн в композитных материалах: жирные кривые и черные точки $(\varkappa<0)$

ляют концы отрезков, соответствующих допустимым разрывам, на априорно эволюционной части ударной адиабаты. На рис. 3.10 a на графике ударной адиабаты и на рис. 3.10 b на диаграмме эволюционности жирными линиями 
и черными точками отмечены возможные состояния за допустимыми разрывами при начальном состоянии $A$. Эволюционные отрезки $A H$ и $L D$ ударной адиабаты разбиваются на участки, причем часть из них не отвечает допустимым разрывам.

При увеличении значения $m / \mu$ число отдельных точек и отрезков ударной адиабаты, соответствующих допустимым разрывам, увеличивается.

В рассмотренных выше случаях координаты точки $A$ выбраны так, что эта точка находится вблизи оси $u_{1}$. Изучение картины интегральных кривых при увеличении координаты $u_{2}$ точки $A$ показало, что наличие отдельных точек $C_{i}$ возможно только при существенном уменьшении значения параметра $\mu / m$.

Отметим, что в противоположность изучаемому случаю, при $m=0$, как было показано в $\S 2$, все априорно эволюционные разрывы оказались допустимыми.

3.2. Неединственность решений автомодельной волновой задачи. Проведенное в п. 3.1 исследование структуры разрывов конкретизирует крупномасштабную гиперболическую модель, состоящую из системы (1.3) и множества допустимых разрывов, определенных как множество разрывов со стационарной структурой. Покажем, что эта гиперболическая модель не гарантирует единственности решений. Неединственность возникает вследствие присутствия на ударной адиабате множества изолированных точек, которые отвечают особым разрывам.

В рамках рассматриваемой модели, описывающей нелинейные волны в композитных средах, для некоторого класса начальных условий решение автомодельной волновой задачи совпадает с решением аналогичной задачи, описываемой той же системой гиперболических уравнений (1.3) в случае, рассмотренном в $\S 1$, когда множество допустимых разрывов определяется условием априорной эволюционности. Тем не менее, следует подчеркнуть, что в некоторых областях начальных параметров решение в рамках рассматриваемой в данной работе модели может быть построено только с использованием особых разрывов и не совпадает с решением, полученным в $\S 1$.

Рассмотрим пример построения решения автомодельной волновой задачи с использованием особых разрывов. Задача о распаде произвольного разрыва для системы уравнений (3.1) формулируется следующим образом: начальные условия $(t=0)$ при $x>0$ соответствуют координатам точки $A$ на плоскости $\left(u_{1}, u_{2}\right)$, а при $x<0$ - координатам точки $P$ (рис. $3.5 a, 3.10 a$ ). Быстрые ударные волны или волны Римана имеют бо́льшую скорость, чем особый разрыв, и могут в автомодельной задаче распространяться перед особым разрывом, а медленные волны соответственно позади него.

Одно из решений сформулированной задачи (для сред с $\varkappa>0$ ) представляет собой следующую последовательность волн: впереди по оси $x$ распространяется быстрая простая волна $A \rightarrow A_{P}$ (рис. $3.5 a$ ), которая переводит состояние $A$ в такую точку $A_{P}$, что состояние за особым разрывом $A_{P} \rightarrow C_{P}$ характеризуется точкой $C_{P}$, представляющей состояние перед медленной ударной волной $C_{P} \rightarrow P$, распространяющейся за особым разрывом $A_{P} \rightarrow C_{P}$. Очевидно, что 
подобных решений имеется столько же, сколько выделенных точек $C_{i}$ на ударной адиабате, соответствующей начальной точке $A$ (в рассматриваемом случае таких точек пять).

Для сред с $\varkappa<0$ пример построения сформулированной задачи о распаде произвольного разрыва состоит в следующей последовательности волн: впереди по оси $x$ идет быстрая ударная волна $A \rightarrow A_{P}$ (рис. $3.10 a$ ), затем особый разрыв $A_{P} \rightarrow C_{P}$ и, наконец, медленная ударная волна $C_{P} \rightarrow P$. Число подобных решений, как и в предыдущем случае, зависит от отношения $\mathrm{m} / \mu$ и в рассматриваемом случае равно семи.

Отметим, что в отличие от разрывов, удовлетворяющих условиям априорной эволюционности, следующих из предположения об отсутствии дополнительных соотношений на разрывах, особые разрывы могут следовать один за другим, так как по состоянию за особым разрывом с меньшей скоростью может распространяться другой особый разрыв. Поэтому наряду с описанными выше могут существовать другие решения, содержащие последовательности особых разрывов.

Качественный анализ решений показывает, что при заданном начальном состоянии справа и различных состояниях слева от разрыва, характеризующихся значениями $u_{1}$ и $u_{2}$, существует конечная область граничных значений на плоскости $\left(u_{1}, u_{2}\right)$, для которых решение автомодельной задачи неединственно. Наличие множества допустимых особых разрывов приводит к множественной неединственности решений задачи. Более детальное исследование решений и вопроса отбора решений в области неединственности проводится ниже на основе численного решения доопределенной задачи о распаде произвольного разрыва для системы уравнений (3.1).

\section{3. Решения полной системы уравнений в частных производных} и их асимптотики. Рассматривается следующая задача. Для системы уравнений (3.1) начальные условия при $t=0$ задаются в виде

$$
u_{\alpha}= \begin{cases}u_{\alpha}^{\mathrm{l}} & \text { при } x<-a, \\ u_{\alpha}^{0}(x) & \text { при }-a \leqslant x \leqslant a, \quad \alpha=1,2, \\ u_{\alpha}^{\mathrm{r}} & \text { при } x>a, \\ & a, u_{\alpha}^{\mathrm{l}}, u_{\alpha}^{\mathrm{r}}=\text { const. }\end{cases}
$$

Разыскивается асимптотика решения при $t \rightarrow \infty$. Если поведение решения таково, что оно в переменных $x / t, t$ при $t \rightarrow \infty$ стремится к некоторому автомодельному решению, зависящему от $x / t$, то это решение будем называть доопределенным решением задачи о распаде произвольного разрыва для гиперболической модели нелинейной теории упругости. Доопределение в задаче о распаде дается конкретной формой правых (негиперболических) частей уравнений $(3.1)$ и функциями $u_{\alpha}^{0}(x)$, задающими сглаживание ступеньки при задании начальных условий. Как будет показано ниже, конкретное задание функций $u_{\alpha}^{0}(x)$ в задаче $(3.1),(3.3)$ определяет решение задачи и его стремление к той или иной автомодельной асимптотике при $t \rightarrow \infty$, когда асимптотика неединственна. 
Ниже приводятся результаты численного решения задач для уравнений (3.1) при различном задании начальных условий (3.3) [47], [48].

При проведении расчетов значения $u_{\alpha}^{\mathrm{r}}$ и $u_{\alpha}^{\mathrm{l}}$ задавались в качестве граничных условий достаточно далеко от области формирования решения.

До проведения расчетов сформулированных выше задач численно было проведено исследование устойчивости стационарных структур и, в частности, структур особых разрывов по отношению к различным одномерным возмущениям. С этой целью решались следующие начально-краевые задачи для системы (3.1): начальные условия задавались в виде $u_{\alpha}^{*}(x)+\delta u_{\alpha}(x)$, причем функции $u_{\alpha}^{*}(x)$ представляли структуру исследуемых разрывов, а $\delta u_{\alpha}(x)$ - возмущение, действие которого изучалось. В зависимости от амплитуды и ширины возмущения решение в виде стационарной структуры разрывов (в том числе особых) восстанавливалось с ростом времени либо распадалось на систему волн. Численные эксперименты показали, что стационарные структуры разрывов устойчивы по отношению к возмущениям не только малой, но и конечной амплитуды. В случае, когда возмущения были так велики, что происходил распад исходной волны, с течением времени формировалось решение, состоящее из последовательности волн: быстрая простая или ударная волна, особый разрыв типа $A_{P} \rightarrow C_{1}$ и движущаяся с меньшей скоростью медленная простая или ударная волна. Волна $A \rightarrow C_{1}$ во всех проведенных численных экспериментах восстанавливалась после взаимодействия со всеми рассмотренными возмущениями.

Ниже описаны результаты численного решения задачи (3.1), (3.3) при различных начальных данных и при достаточно больших значениях времени (когда волны успели разойтись). В состав решений входят особые разрывы, обладающие только простыми структурами. Состояния за особыми разрывами будут обозначаться буквами $C$, снабженными дополнительными индексами. Нижний индекс 1 или 2 обозначает тип разрыва, подобный рассмотренным выше разрывам $A \rightarrow C_{1}$ и $A \rightarrow C_{2}$, структуры которых изображены на рис. 3.3 и 3.8 .

Примеры 3.1-3.3 решались при следующих значениях параметров: $m=3$, $\mu=0.05, f=1, g=1, \varkappa=4, u_{1}^{\mathrm{r}}=2, u_{2}^{\mathrm{r}}=0.4$ (точка $A$ на рис. $3.11-3.13$ ), различных значений $u_{\alpha}^{1}$ и различных $u_{\alpha}^{0}(x)$.

ПРимеР 3.1. $u_{1}^{1}=-2, u_{2}^{1}=0.4$ (точка $P$ на рис. $3.11 c$ ), функции $u_{\alpha}^{0}(x)$ представляют собой монотонный переход от значений $u_{\alpha}^{\mathrm{l}}$ к значениям $u_{\alpha}^{\mathrm{r}}$ (схема на рис. 3.11 a).

При достаточно больших значениях времени решение представляет последовательность волн: быстрая простая волна $A \rightarrow A_{P}$ (рис. $3.11 \mathrm{c}$ ), особый разрыв $A_{P} \rightarrow C_{1}$, медленная ударная волна $C_{1} \rightarrow P$. Особый разрыв, входящий в состав решения, имеет простую структуру, изображенную на рис. 3.3 . На рис. 3.11 представлены графики структуры особого разрыва в зависимости от координаты $x$ в фиксированный момент времени $t$ (рис. $3.11 b$ ) и на плоскости $\left(u_{1}, u_{2}\right)$ (рис. $\left.3.11 c\right)$.

ПРимеР 3.2. $u_{1}^{1}=-1.7, u_{2}^{1}=1$ (точка $P$ на рис. $3.12 c$ ), функции $u_{\alpha}^{0}(x)$ представляют монотонный переход от значений $u_{\alpha}^{\mathrm{l}}$ к значениям $u_{\alpha}^{\mathrm{r}}$ (рис. $3.12 a$ ). 


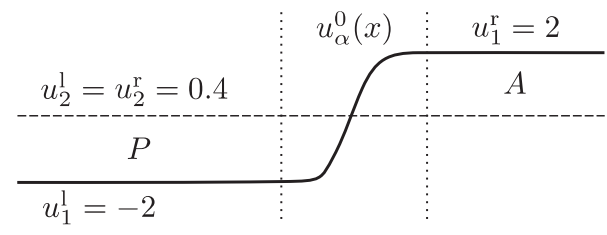

$a$
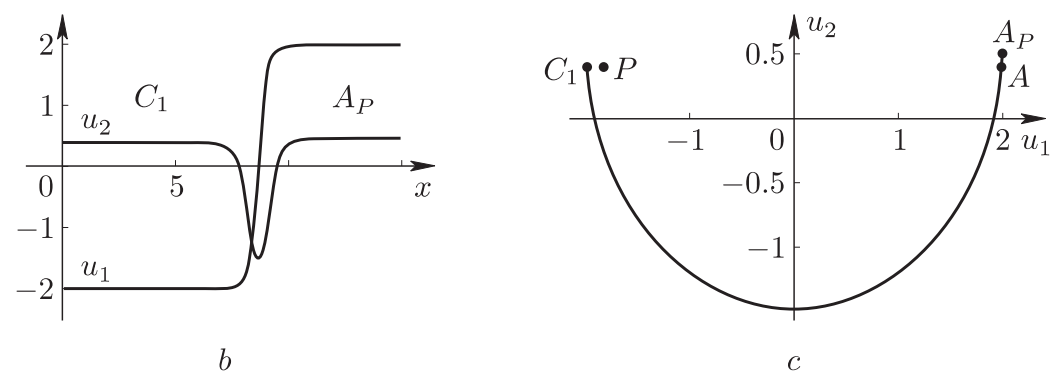

Рис. 3.11. Последовательность волн, представляющих асимптотику примера $3.1(\varkappa>0)$

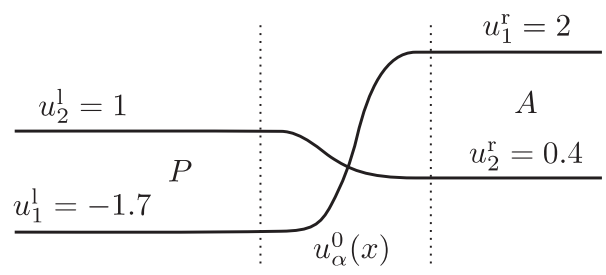

$a$
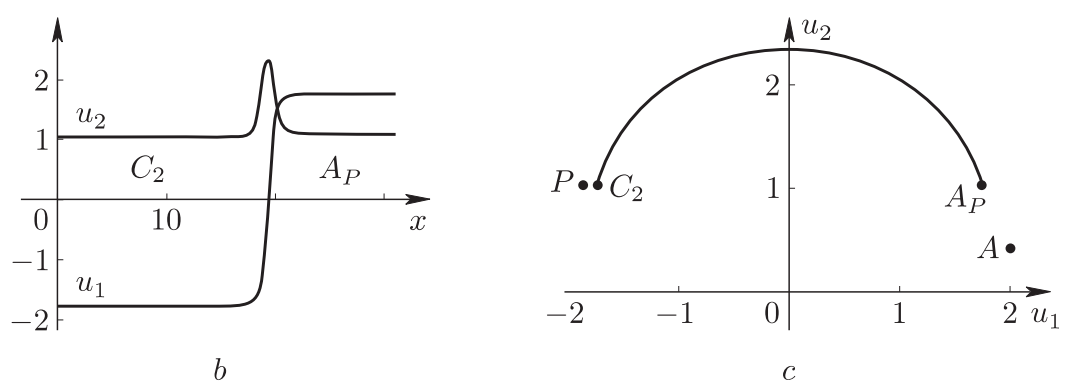

Рис. 3.12. Последовательность волн, представляющих асимптотику примера $3.2(\varkappa>0)$

Решение, представленное на рис. $3.12 b, c$, состоит из последовательности волн: быстрая простая волна $A \rightarrow A_{P}$, особый разрыв $A_{P} \rightarrow C_{2}$, медленная простая волна $C_{2} \rightarrow P$. Структура особого разрыва соответствует простой структуре, изображенной на рис. $3.3 b$. 
ПримеР 3.3. $u_{1}^{1}=-2, u_{2}^{1}=0.4$ (координаты точки $P$ (рис. 3.13) точно такие, как и в примере 3.1$)$, функции $u_{\alpha}^{0}(x)$ представлены на рис. $3.13 a$.

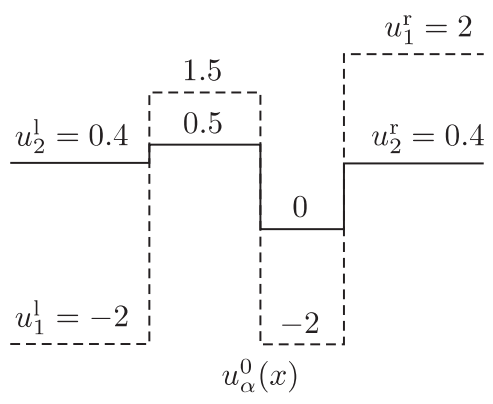

a

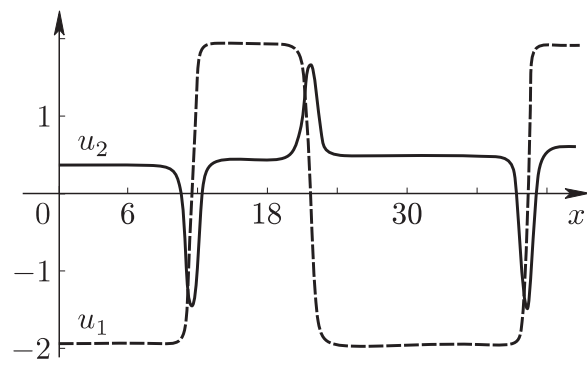

$b$

Рис. 3.13. Последовательность волн, представляющих асимптотику примера $3.3(\varkappa>0)$

Полученная асимптотика представляет собой последовательность волн: быстрая простая волна, последовательность трех особых разрывов с простой структурой и медленная ударная волна. Последовательность трех особых разрывов, имеющих простые структуры, изображена на рис. $3.13 b$.

В примерах 3.1 и 3.3 взяты одинаковые граничные условия, а функции $u_{\alpha}^{0}(x)$ различны. Полученные решения различны и содержат разное число особых разрывов.

Примеры 3.4-3.7 решались при следующих значениях параметров: $m=1.5$, $\mu=0.05, f=1, g=1, \varkappa=-2, u_{1}^{\mathrm{r}}=1, u_{2}^{\mathrm{r}}=0.1$ (точка $A$ на рис. $3.11-3.13$ ), различных значениях $u_{\alpha}^{l}$ и различных $u_{\alpha}^{0}(x)$.

ПримеР 3.4. $u_{1}^{1}=-1.2, u_{2}^{1}=0.4$ (точка $P$ на рис. $3.14 c$ ), функции $u_{\alpha}^{0}(x)$ представляют собой монотонный переход от значений $u_{\alpha}^{\mathrm{l}}$ к значениям $u_{\alpha}^{\mathrm{r}}$ (схема на рис. $3.14 a)$.

На рис. 3.14 с представлено решение при достаточно больших значениях времени на плоскости $\left(u_{1}, u_{2}\right)$ и изменение функций $u_{1}$ и $u_{2}$ в зависимости от координаты $x$ в фиксированный момент времени $t$ (рис. $3.14 b$ ). Решение представляет последовательность волн: быстрая ударная волна $A \rightarrow A_{P}$, особый разрыв $A_{P} \rightarrow C_{1}$, медленная ударная волна $C_{1} \rightarrow P$. Особый разрыв имеет простую структуру, изображенную на рис. 3.8 .

ПримеР 3.5. $u_{1}^{1}=-1.4, u_{2}^{1}=1.4$ (точка $P$ на рис. 3.15 ), функции $u_{\alpha}^{0}(x)$ представляют монотонный переход от значений $u_{\alpha}^{\mathrm{l}}$ к значениям $u_{\alpha}^{\mathrm{r}}$.

Решение, представленное на рис. 3.15, состоит из последовательности волн: быстрая ударная волна $A \rightarrow A_{P}$, особый разрыв $A_{P} \rightarrow C_{2}$, медленная ударная волна $C_{2} \rightarrow P$. Структура особого разрыва соответствует простой структуре, изображенной на рис. 3.8 .

ПримеР 3.6. $u_{1}^{1}=1.4, u_{2}^{1}=1.4$ (точка $P$ на рис. 3.16 ), функции $u_{\alpha}^{0}(x)$ представлены на схеме 3.16 a. Решение, представленное на рис. 3.16 , состоит 


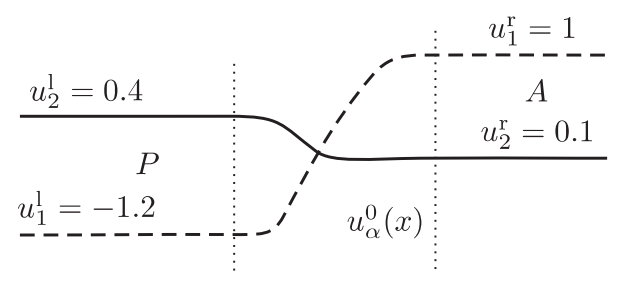

$a$
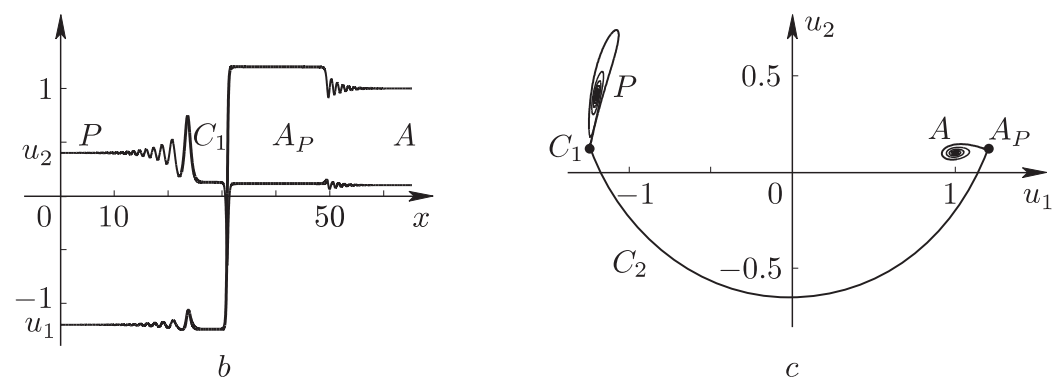

Рис. 3.14. Последовательность волн, представляющих асимптотику примера $3.4(\varkappa<0)$

из последовательности волн: быстрая ударная волна $A \rightarrow A_{P}$, особый разрыв $A_{P} \rightarrow C_{2}$, особый разрыв $C_{2} \rightarrow C_{1}^{\prime}$ (этот особый разрыв из начального состояния, соответствующего точке $C_{2}$, имеет простую структуру, изображенную на рис. 3.8$)$, медленная ударная волна $C_{1}^{\prime} \rightarrow P$.

ПримеР 3.7. $u_{1}^{\mathrm{l}}=-1.2, u_{2}^{\mathrm{l}}=0.4$ (точка $P$ на рис. 3.17 ), функции $u_{\alpha}^{0}(x)$ представлены на схеме рис. 3.17 .

Решение представленное на рис. $3.17 b, c$, состоит из последовательности волн: быстрая ударная волна $A \rightarrow A_{P}$, последовательность трех особых разрывов $A_{P} \rightarrow C_{1}, C_{1} \rightarrow C_{1}^{\prime}, C_{1}^{\prime} \rightarrow C_{1}^{\prime \prime}$ и медленная ударная волна $C_{1}^{\prime \prime} \rightarrow P$. Особые разрывы из начальных состояний $A_{P}, C_{1}, C_{1}^{\prime}$ имеют простую структуру, аналогичную структуре, изображенной на рис. 3.8 .

В примерах 3.4 и 3.7 взяты одинаковые граничные условия, а функции $u_{\alpha}^{0}(x)$ различны. Полученные решения различны и содержат разное число особых разрывов. Сравнение начальных функций в этих примерах иллюстрирует возможность получения асимптотик решений с различным количеством особых разрывов для задач с одинаковыми граничными условиями. Если начальные условия рассматриваемой обобщенной задачи о распаде произвольного разрыва задавать в виде монотонно сглаженной “ступеньки" (как в примерах $3.1,3.2,3.4,3.5)$, то решение либо не содержит особых разрывов, либо содержит один из особых разрывов $A_{P} \rightarrow C_{1}$ или $A_{P} \rightarrow C_{2}$ с простейшими структурами, которые изображены на рис. $3.3(\varkappa>0)$ и рис. $3.8(\varkappa<0)$. Отметим, что даже в том случае, когда правое и левое граничное условие расположены в одной координатной четверти и возможно решение без особых разры- 

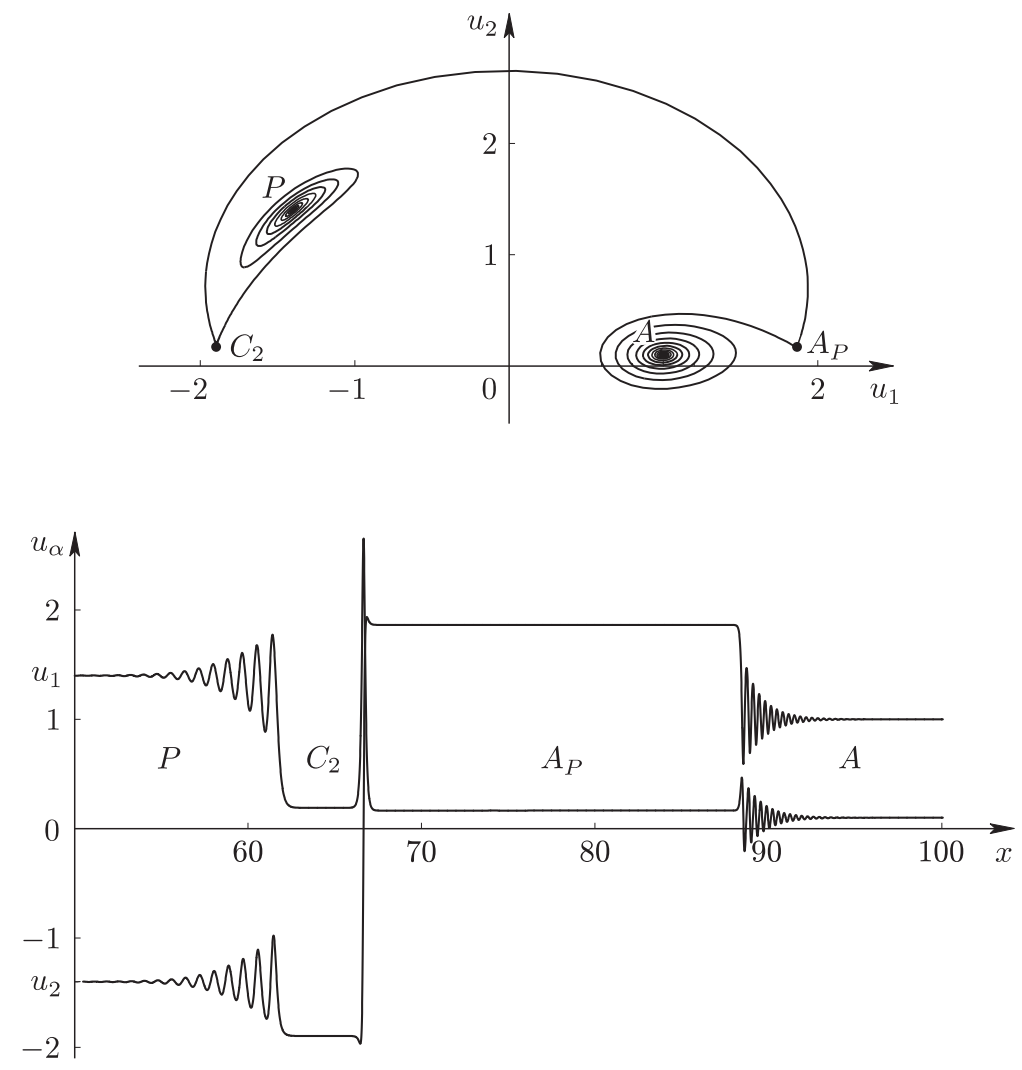

Рис. 3.15. Последовательность волн, представляющих асимптотику примера $3.5(\varkappa<0)$

вов, тем не менее решение может содержать последовательность особых разрывов, если функции $u_{\alpha}^{0}(x)$ не монотонны - что иллюстрирует пример 3.6.

Численные эксперименты, проведенные для других наборов параметров $m$, $\mu, u_{\alpha}^{\mathrm{r}}, u_{\alpha}^{\mathrm{l}}$, показали, что отмеченные в предыдущем абзаце закономерности сохраняются.

Для описанной модели имеет место подобие нелинейных явлений, если сохраняются отношения величин $m / \mu$ и начальные условия, рассматриваемые как функции $x / \sqrt{\mu}$ (или если задаются граничные условия, то $t / \sqrt{\mu}$ ). Поэтому полученные и описанные выше результаты могут рассматриваться как решения тех же уравнений с другим набором параметров.

3.4. Выводы. Таким образом, выявлено, что при существенном влиянии дисперсии в мелкомасштабных явлениях $(m / \mu \gg 1)$ множество допустимых разрывов, имеющих одномерную стационарную структуру, приобретает сложное строение. На ударной адиабате там, где выполнены условия априорной эволюционности, появляются отрезки, не соответствующие допустимым разрывам. Одновременно на априорно неэволюционных отрезках ударной адиаба- 


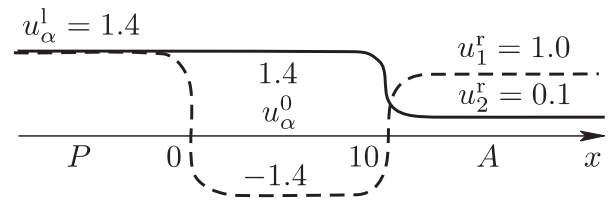

a

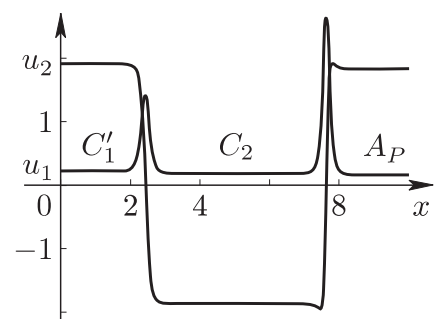

b

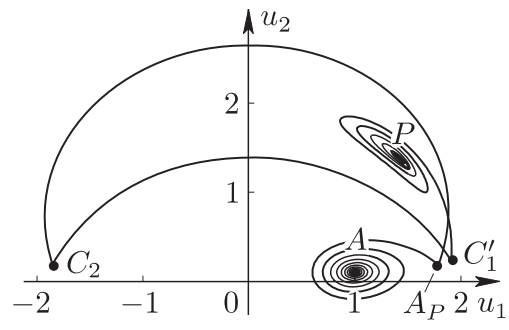

c

Рис. 3.16. Последовательность волн, представляющих асимптотику примера $3.6(\varkappa<0)$

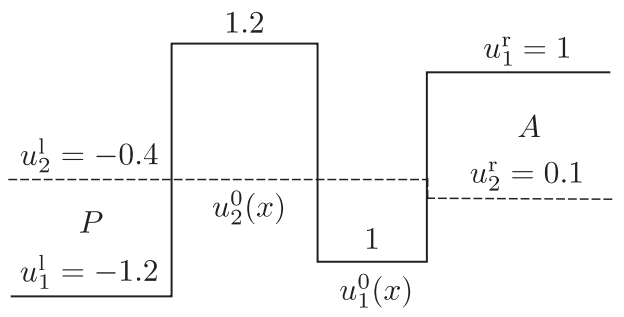

$a$

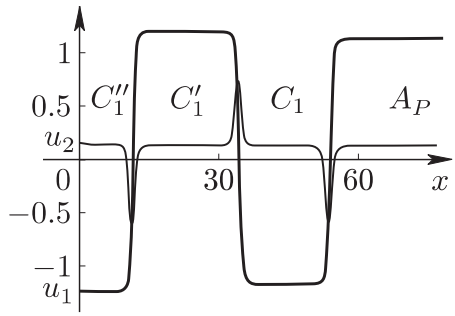

$b$

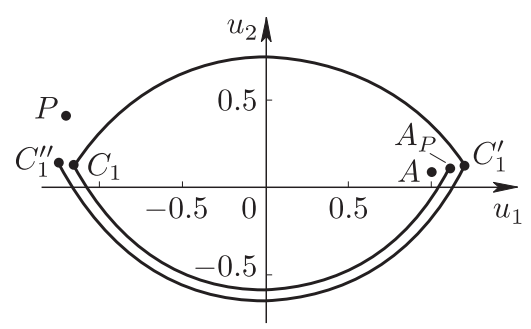

c

Рис. 3.17. Последовательность волн, представляющих асимптотику примера $3.7(\varkappa<0)$

ты появляются точки, соответствующие допустимым разрывам. Число и тех и других зависит от величины $m / \mu$ и растет с ростом этого отношения.

Гиперболическая модель, основанная на дифференциальных уравнениях нелинейной теории упругости и множестве допустимых разрывов, имеющих 
структуру при учете эффектов дисперсии и диссипации, при большом относительном влиянии дисперсии $(m / \mu \gg 1)$ позволяет построить много решений одной и той же автомодельной задачи.

Численное решение неавтомодельных задач для уравнений в частных производных, учитывающих дисперсию и диссипацию в случаях, когда постановка задачи такова, что с ростом времени решение должно выходить на автомодельную асимптотику, показало, что асимптотика может быть различной в зависимости от деталей постановки неавтомодельной задачи, исчезающих в автомодельной постановке. При заданном отношении $m / \mu$ на характер асимптотики решения влияет вид функций, задающих сглаживание начального разрыва. Из проделанных расчетов следует, что при задании различным образом этих функций наиболее часто возникают решения, содержащие особые разрывы с наименьшими скоростями и простейшими структурами. Решения, содержащие разрывы других типов с наиболее сложными структурами, также могут осуществляться, поскольку эти структуры оказались устойчивыми. Для их осуществления нужно более специальное задание функций, задающих сглаживание начального разрыва.

\section{§4. Неклассические разрывы при распространении продольных волн в вязко-упругих стержнях со сложной нелинейностью}

В предыдущих параграфах, посвященных нелинейным квазипоперечным волнам в упругих средах при наличии мелкомасштабных эффектов дисперсии и диссипации, показано, что сложное строение множества допустимых ударных волн обусловлено помимо наличия дисперсии и диссипации также некоторыми свойствами гиперболической части уравнений. А именно, нужно, чтобы состояние за разрывом, распространяющимся по заданному состоянию с заданной скоростью, не определялось однозначно соотношениями, следующими из законов сохранения, а было как минимум трехзначным. Это свойство является случаем общего положения для достаточно сложных систем уравнений.

При распространении продольных волн в упругой среде можно ожидать сложного поведения ударных волн и многозначных решений автомодельных задач только в случае сложной нелинейной зависимости между напряжением и деформацией. Ниже рассматривается простая модель [22], [45], [46], [50] для описания продольных волн в стержнях, обладающая требуемым свойством.

4.1. Разрывы со стационарной структурой. Задача о распаде произвольного разрыва. Если изучать умеренно длинные волны в вязко-упругом стержне, то продольную деформацию $u=\partial w / \partial x(w-$ продольное перемещение точек стержня) можно приближенно рассматривать как функцию $x$ и $t$, а суммарное по сечению напряжение стержня $\sigma$-как функцию $u$. Уравнение, описывающее в этом случае распространение продольных волн, имеет следующий вид (см. [46]):

$$
\frac{\partial^{2} u}{\partial t^{2}}=\frac{\partial^{2} \sigma(u)}{\partial x^{2}}+\beta^{2} \frac{\partial^{4} u}{\partial t^{2} \partial x^{2}}+\alpha \frac{\partial^{3} u}{\partial t \partial x^{2}} .
$$

Здесь $x$ - лагранжева координата. Член с $\beta^{2}$ представляет главное проявление дисперсии, возникающей за счет слабой неодномерности движений. 
Для круглых стержней $\beta=\nu r$, где $\nu$ - коэффициент Пуассона, $r$ - радиус стержня [51]. Коэффициенты $\alpha$ и $\beta$ для волн малой амплитуды принимаются постоянными.

При малой нелинейности из уравнения (4.1) можно получить независимые уравнения для волн, распространяющихся в каждую сторону, как это делается при выводе уравнения Бюргерса или Кортевега-де Вриза. Для волн, распространяющихся в положительном направлении оси $x$, уравнение имеет вид:

$$
\begin{gathered}
\frac{\partial u}{\partial t}+\frac{\partial \varphi(u)}{\partial x}=\mu \frac{\partial^{2} u}{\partial x^{2}}-m \frac{\partial^{3} u}{\partial x^{3}} \\
\mu=\frac{1}{2} \alpha, \quad m=\frac{1}{2} c_{0} \beta^{2}, \quad \varphi(u)=\int c(u) d u, \quad c(u)=\sqrt{\frac{d \sigma}{d u}} .
\end{gathered}
$$

Здесь $c_{0}(u)$ - некоторое среднее значение $c$ в интервале изменения $u$. Для дальнейшего исследования будут представлять интерес функции $\varphi(u)$, имеющие две точки перегиба. При этом функция $\sigma(u)$ должна также иметь две точки перегиба, как это показано на рис. 4.1.

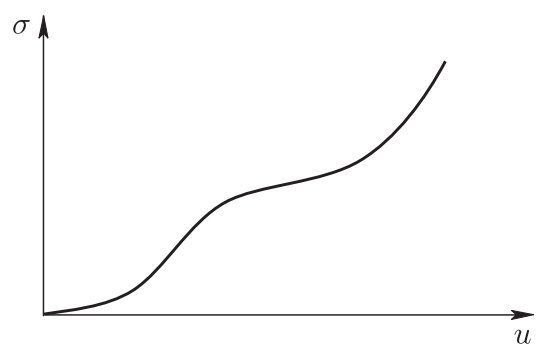

Рис. 4.1. Качественный вид функции $\sigma(u)$

При распространении очень длинных волн оба члена в правой части становятся малыми по сравнению с членами в левой части и при пренебрежении правой частью уравнение (4.2) приобретает вид

$$
\frac{\partial u}{\partial t}+c(u) \frac{\partial u}{\partial x}=0, \quad c(u)=\frac{\partial \varphi(u)}{\partial u} .
$$

Здесь $c(u)$ - характеристическая скорость. Заметим, что при преобразовании Галилея к $c(u)$ прибавляется постоянная, а функция $\varphi(u)$ приобретает линейное по $u$ слагаемое. Уравнение (4.3) допускает разрывные решения. Это уравнение (так же как и (4.2)) выражает закон сохранения, поэтому соответствующее соотношение на разрыве можно записать в виде

$$
W=\frac{[\varphi(u)]}{[u]} .
$$

Здесь $W$ - скорость разрыва, квадратными скобками обозначена разность значений функций за и перед разрывом.

Неединственность решений автомодельных задач для уравнения (4.3) с использованием разрывов со стационарной структурой имеет место, если график 
функции $\varphi(u)$ имеет не менее двух точек перегиба. В качестве функции $\varphi(u)$ при расчетах, результаты которых излагаются ниже, взята функция

$$
\varphi(u)=u^{4}-u^{2} .
$$

График этой функции изображен на рис. 4.2 .

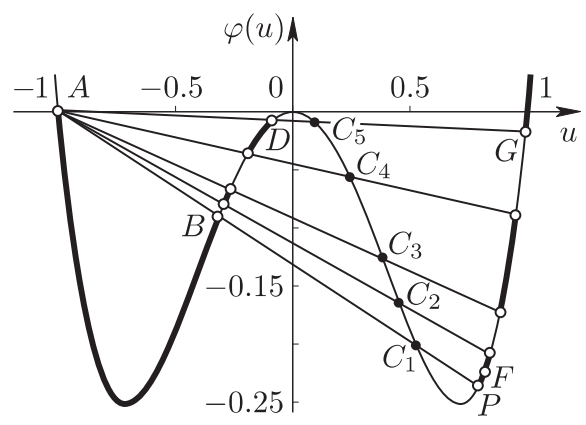

$a$

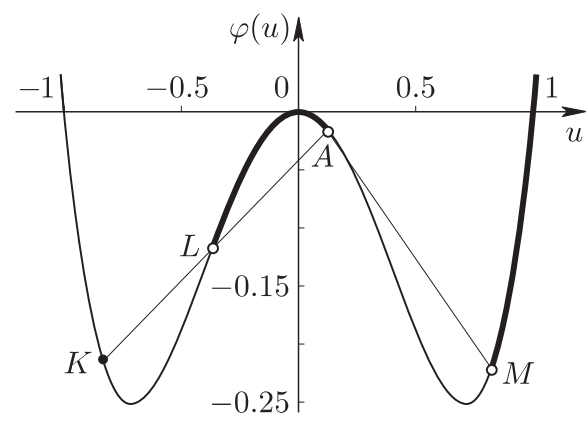

$b$

Рис. 4.2. График функции $\varphi(u)=u^{4}-u^{2}$

Стационарная структура разрывов описывается следующими равенствами:

$$
\begin{gathered}
u=u(\xi), \quad \xi=-x+W t, \\
m \frac{d^{2} u}{d \xi^{2}}+\mu \frac{d u}{d \xi}=F(u), \quad F(u)=W\left(u-u^{\mathrm{R}}\right)-\left(\varphi(u)-\varphi\left(u^{\mathrm{R}}\right)\right), \\
\lim _{\xi \rightarrow \infty} u(\xi)=u^{\mathrm{R}}, \quad \lim _{\xi \rightarrow-\infty} u(\xi)=u^{\mathrm{L}} .
\end{gathered}
$$

Если такое решение существует, то с точки зрения большого масштаба длины оно должно представлять собой разрыв со стационарной структурой, в котором $u=u^{\mathrm{R}}$ и $u=u^{\mathrm{L}}-$ состояния перед $(x>0)$ и за $(x<0)$ разрывом. Нетрудно убедиться, что состояния $u^{\mathrm{L}}$ и $u^{\mathrm{R}}$ удовлетворяют соотношениям (4.4) и представляют, таким образом, состояния за и перед разрывом. Разрывы, которым соответствует решение задачи о структуре, будем называть допустимыми. Состояние перед разрывом будем обозначать буквой $A$ на графике функции $\varphi(u)$ (рис. 4.2).

Путем качественного анализа, использовавшего аналогию уравнения (4.6) и уравнения движения материальной точки, было показано [45], что при достаточно большом значении $\mathrm{m} / \mu^{2}$ множество состояний $u^{\mathrm{L}}$ за допустимыми разрывами при заданном $u^{\mathrm{R}}$ состоит из отрезков и отдельных точек оси $u$. Для удобства это множество на рис. 4.2 a изображено на графике функции $\varphi(u)$ в виде жирных отрезков и отдельных точек $C_{i}(i=1, \ldots, 5)$. Оно было рассчитано для параметров $u^{\mathrm{R}}=-1, m=1.3, \mu=0.05$. Будем называть разрыв $A \rightarrow C_{i}$ особым, если $A$ - состояние перед разрывом, а $C_{i}$ - выделенные отдельные точки, представляющие состояния за разрывом, для которых существует решение задачи (4.6) о структуре разрыва. Число и расположение отрезков и отдельных точек, представляющих состояния за допустимыми разрывами при заданном состоянии перед ними, зависят от параметра $m / \mu^{2}$. При 
увеличении этого параметра, когда $u^{\mathrm{R}}$ фиксировано, число отдельных точек и отрезков увеличивается.

Если точку $A$ перемещать, то качественных изменений множества возможных состояний за разрывом происходить не будет, пока точка $A$ не перейдет через точку перегиба графика функции $\varphi(u)$. Множество допустимых разрывов для начальной точки $A$, лежащей между двумя точками перегиба, изображено на рис. $4.2 b$ и состоит из точки $K$ и отрезков $[A, L],[M, \infty]$. Точка $M$ лежит на продолжении касательной к графику функции $\varphi(u)$ в точке $A$, а положение отдельной точки $K$ и точки $L$ зависит от $m / \mu^{2}$. Если точка $A$ лежит правее обеих точек перегиба графика функции $\varphi(u)$, то множество состояний за возможными разрывами представляется полубесконечным интервалом, примыкающим к точке $A$ справа.

Уравнение (4.3) допускает непрерывные расширяющиеся со временем решения $u(x, t)$, принимающие постоянные значения на веере расходящихся характеристик на плоскости $(x, t)$. В этих решениях величина $\varphi^{\prime}(u)=c(u)$ должна убывать по модулю с ростом $t$. Это означает, что со временем $u$ растет, если $\varphi^{\prime \prime}(u)<0$, и убывает при $\varphi^{\prime \prime}(u)>0$.

Рассмотрим автомодельные решения уравнения (4.3), считая, что они могут содержать описанные выше разрывы со стационарной структурой. Будем считать, что при $t=0$ начальные данные имеют вид $u=u^{\mathrm{L}}$ при $x<0$ и $u=u^{\mathrm{R}}$ при $x>0$. В дальнейшем будем рассматривать случай $u^{\mathrm{R}}=-1$.

Пусть сначала состояние $u^{\mathrm{L}}$ при $x<0$ задано точкой $F$ (рис. $4.2 a, m=1.3$, $\mu=0.05)$, тогда задача о распаде разрыва для уравнения (4.3) имеет несколько решений. Одно из них представляется скачком $A \rightarrow F$. Остальные решения состоят из двух скачков $A \rightarrow C_{i}$ и $C_{i} \rightarrow F(i=2, \ldots, 5)$. Скорости этих разрывов, определяемые соотношением (4.4), таковы, что для $i=2, \ldots, 5$ разрыв $A \rightarrow C_{i}$ движется быстрее разрыва $C_{i} \rightarrow F$ (заметим, что для $i=1$ это условие нарушается). Если точку $F$ двигать по графику вверх направо, то последовательно будут исчезать некоторые из перечисленных решений, пока не останется одно, представляемое скачком $A \rightarrow F$, когда точка $F$ будет расположена выше точки $G$.

Если точку $F$, представляющую состояние при $x=-\infty$, передвигать по графику функции $\varphi(u)$ налево (левее точки $P$, рис. $4.2 a$ ), то будет возможно еще одно решение, состоящее из двух разрывов $A \rightarrow C_{1}, C_{1} \rightarrow F$, однако решение $A \rightarrow F$ пропадет. При дальнейшем движении точки $F$ по кривой функции $\varphi(u)$ налево выше точки $C_{1}$ разрыв $C_{1} \rightarrow F$ заменится на непрерывную волну, в которой изменения $u$ и $\varphi(u)$ происходят от точки $C_{1}$ до точки $F$ или до точки, из которой можно провести касательную в точку $F$, представляющую скачок (имеющий стационарную структуру) из точки касания в точку $F$. При уменьшении $u^{\mathrm{L}}$ некоторые из ударных волн $C_{i} \rightarrow F$ заменяются сначала непрерывной волной, а затем сложными волнами, которые состоят из непрерывной расширяющейся со временем волны, начинающейся из состояния, соответствующего точке $C_{i}$, и примыкающего к нему разрыва с конечным состоянием, соответствующим точке $F$.

Если точка $F$ принадлежит отрезку $D P$, то существует пять решений, состоящих из разрыва $A \rightarrow C_{i}(i=1, \ldots, 5)$ и перехода $C_{i} \rightarrow F$, который пред- 
ставляется либо разрывом, либо непрерывной волной, либо сложной волной, содержащей разрыв и непрерывную волну.

Когда точка $F$ переходит через точку $D$, двигаясь по графику функции $\varphi(u)$ влево, то сначала прекращает существование решение $A \rightarrow C_{5}, C_{5} \rightarrow F$, поскольку скорость разрыва $C_{5} \rightarrow F$ будет иметь скорость меньшую, чем разрыв $A \rightarrow C_{5}$. Далее при движении точки $F$ к точке $B$ последовательно исчезают решения, связанные с точками $C_{4}, C_{3}, C_{2}$ и $C_{1}$. Если точка $F$ принадлежит отрезку $A B$, то имеется одно решение, представляемое разрывом $A \rightarrow F$. В случае $u^{\mathrm{L}}<-1$ имеется также единственное решение в виде непрерывной волны.

Таким образом, для описанного выше множества значений $u^{\mathrm{L}}$ решение задачи о распаде разрыва неединственно, причем для данных значений параметров $m$ и $\mu$ число возможных решений (которые строятся с использованием разрывов, имеющих структуру) в случае неединственности достигает пяти.

В случае неединственности с целью выбора решения ниже будут рассматриваться нестационарные решения уравнения (4.2), которые должны иметь автомодельные асимптотики, описываемые уравнениями (4.3) и (4.4), когда пространственные масштабы решения становятся большими.

\section{2. Численное исследование задач с автомодельной асимптоти-} кой. Для численного моделирования нестационарных процессов, описываемых уравнением (4.2), начальные данные будем задавать не в виде кусочно постоянной функции (ступеньки), а в виде непрерывно изменяющейся на некотором отрезке функции (“сглаженной" ступеньки).

$$
\begin{gathered}
u= \begin{cases}u^{\mathrm{l}} & \text { при } x<-a, \\
u^{0}(x) & \text { при }-a \leqslant x \leqslant a, \\
u^{\mathrm{r}} & \text { при } a<x,\end{cases} \\
\quad a, u^{\mathrm{l}}, u^{\mathrm{r}}=\text { const. }
\end{gathered}
$$

Заметим, что умножением $x$ и $t$ на одно и то же число параметр $\mu$ в уравнении (4.2) можно обратить в единицу, тогда решение задачи будет зависеть от функции, задающей начальное условие, и одного параметра, входящего в преобразованное уравнение и выражающегося через значения коэффициентов до преобразования в виде $m / \mu^{2}$.

Приведем теперь результаты численных экспериментов [50]. При расчетах значения $u^{\mathrm{r}}$ и $u^{\mathrm{l}}$ задавались в качестве граничных условий достаточно далеко от области формирования решения задачи о распаде произвольного разрыва.

Получены следующие результаты.

а) Численно найдены стационарные структуры как решения обыкновенного дифференциального уравнения (4.6). Выявлены и рассчитаны структуры волн $A \rightarrow C_{i}(i=1, \ldots, 5)$. На рис. 4.3 приведены графики решений для отдельных точек $C_{i}$ и значений параметров $m=1.3, \mu=0.05$. На этих графиках видно, что структура решений для каждой из выделенных точек $C_{i}$ различна, причем число колебаний внутри структуры увеличивается с увеличением индекса точки. Самую простую структуру имеет волна, состояние за которой определяется точкой $C_{1}$. Положение и величины выделенных на рис. $4.2 a$ отрезков, 
в которых существует решение в виде стационарной структуры, также найдено численно.
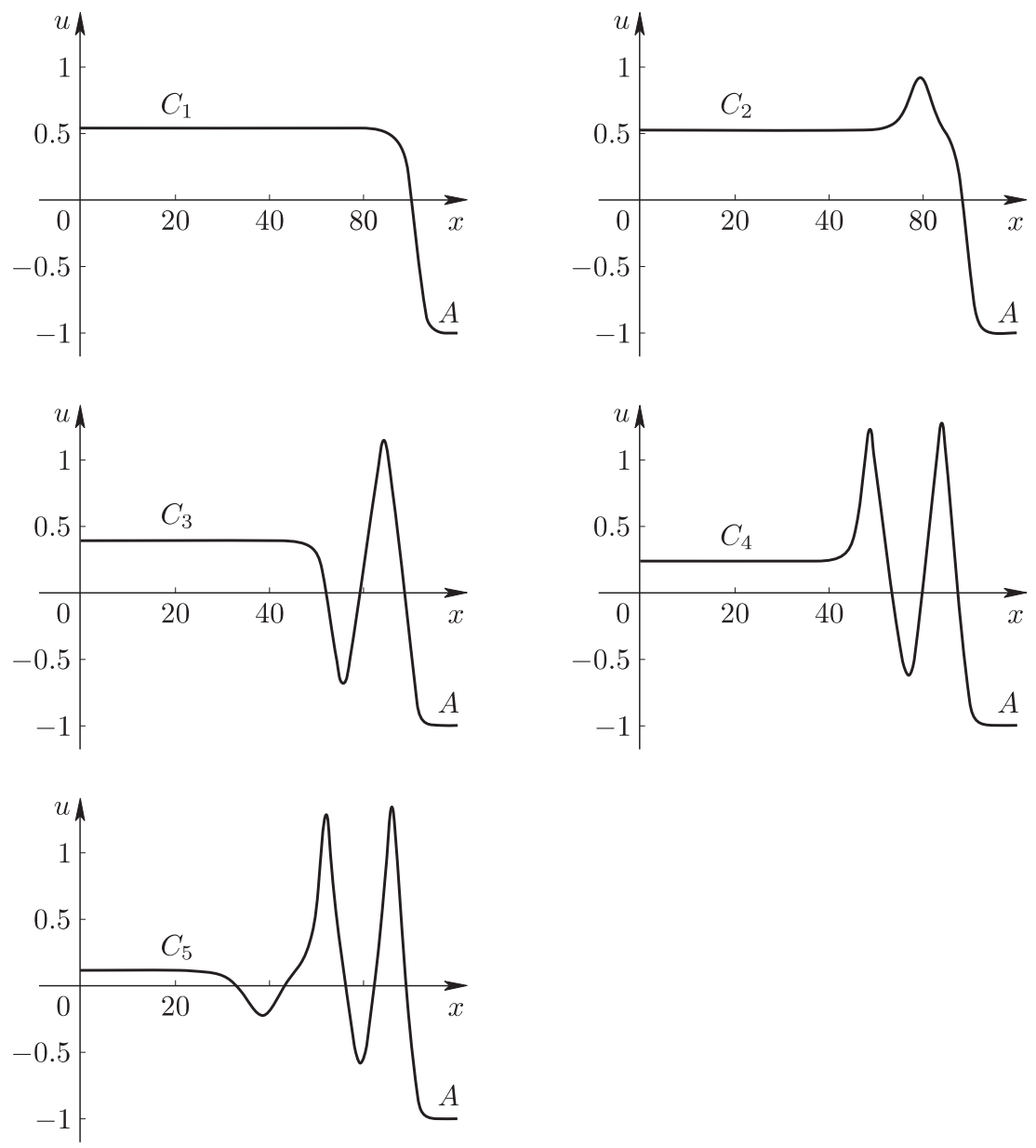

Рис. 4.3. Стационарные структуры

b) Проведено исследование устойчивости стационарных структур и, в частности, структур особых разрывов по отношению к различным возмущениям. Для этого решались следующие начально-краевые задачи для уравнения (4.2): правое граничное условие задавалось в виде $u^{\mathrm{r}}=-1$ (при $x=0, t \geqslant 0$ ), левое граничное условие - в виде $u^{1}=C_{i}$ (при $\left.x=x_{L}, t \geqslant 0\right)$, в качестве начального условия $(t=0)$ выбрана функция $u=u^{*}(x)+\delta u(x)$, причем $u^{*}(x)$ представляет решение обыкновенного дифференциального уравнения (4.6) для данного начального значения $u=u^{l}$, соответствующего одной из точек $C_{i}$. Кроме того, произвольные различные возмущения задавались перед исследуемой волной, т. е. при больших значениях $x$, где $u^{*}$ практически не отличается от -1 . Решались также задачи, когда $\delta(x)=0$, а внесены возмущения в правое граничное условие $u^{\mathrm{r}}=-1+\delta u(t)$. Во всех перечисленных задачах в последующие моменты времени $(t>0)$ возмущение распространялось и взаимодействовало 
со структурой особого разрыва. В зависимости от амплитуды и ширины возмущения решение в виде стационарной структуры восстанавливалось с ростом времени, либо распадалось на систему волн. Численные эксперименты показали, что стационарные волны, представляющие структуру особого разрыва, устойчивы по отношению к возмущениям не только малой, но также и конечной амплитуды. В случае, когда возмущения были так велики, что происходил распад исходной волны, с течением времени формировалось решение, состоящее из следующей последовательности волн: волна $A \rightarrow C_{1}$, далее движущаяся с меньшей скоростью простая волна, в которой величины меняются от точки $C_{1}$ до некоторой точки такой, что касательная, проведенная к графику функции $\varphi(u)$ в этой точке, проходит через точку $C_{i}$, и затем ударная волна в точку $C_{i}$. Только волна $A \rightarrow C_{1}$ во всех проведенных численных экспериментах восстанавливалась после взаимодействия с любыми возмущениями.

Аналогичным способом численно исследовалась устойчивость неособых разрывов с конечными состояниями, принадлежащими отрезкам ударной адиабаты. Все эти разрывы оказались устойчивыми по отношению к не слишком большим возмущениям. При действии больших возмущений некоторые разрывы приобретали структуру, периодически зависящую от времени.

с) Численно решены аналоги задачи о распаде произвольного разрыва (4.7). Начально-краевая задача для уравнения (4.2) формулируется следующем образом: левое граничное условие $u^{1}=u_{F}$ (при $\left.x=0, t \geqslant 0\right)$, правое граничное условие $u^{\mathrm{r}}=-1$ (при достаточно большом $x=x_{R}, t \geqslant 0$ ), начальные данные $u=u_{0}(x)$ выбирались так, что $u_{0}(x)=-1$, если $x>a$, и $u_{0}(x)=u_{F}$, если $x<b$.

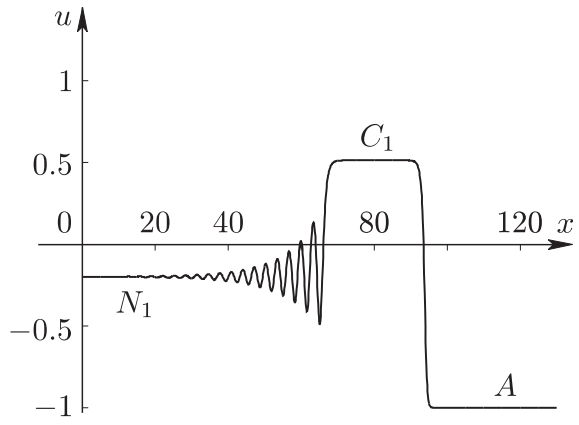

$a$

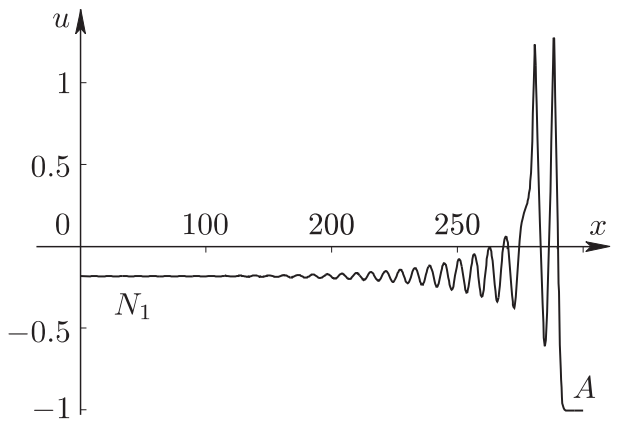

$b$

Рис. 4.4. Асимптотики решений уравнений в частных производных

Оказалось, что если $u_{B}<u_{F}<u_{P}$ (здесь $u_{B}$ и $u_{P}-$ координаты точек $B$ и $P$ рис. $4.2 a)$, то при монотонных функциях $u_{0}(x)$ решение при $t \rightarrow \infty$ всегда стремится к одному из автомодельных решений, описанных в п. 4.1, а именно к системе волн с волной $A \rightarrow C_{1}$, идущей первой. График этого решения в некоторый момент времени представлен на рис. 4.4 а для случая, когда точка с координатами $\left(u^{\mathrm{l}}, \varphi\left(u^{\mathrm{l}}\right)\right)$ принадлежит отрезку графика функции $\varphi(u)$, примыкающему к точке $D$ слева.

$\mathrm{K}$ этому типу решений относятся описанные выше решения, возникающие при распаде волн $A \rightarrow C_{i}(i=2, \ldots, 5)$. На рис. $4.4 b$ представлен график 
решения этой же начально-краевой задачи, но в качестве начальных данных задана функция, близкая к решению обыкновенного дифференциального уравнения (4.6) в том случае, когда правое граничное условие выбрано так же, как в случае, изображенном на рис. 4.4 a. Это решение с увеличением времени стремится к стационарной структуре. Таким образом, продемонстрировано, что вид решения зависит от того, какая функция задает начальные условия.

Если $u_{P}<u_{F}<u_{G}$ (рис. $4.2 a$ ) и сглаживающая функция монотонна, то в результате эволюции решения при больших временах образуется одна волна с периодической нестационарной структурой.

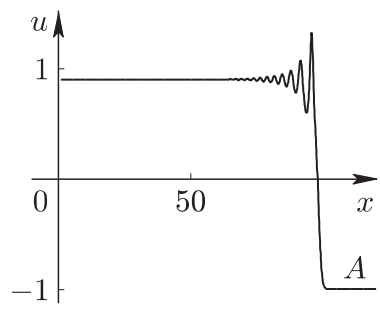

$a$
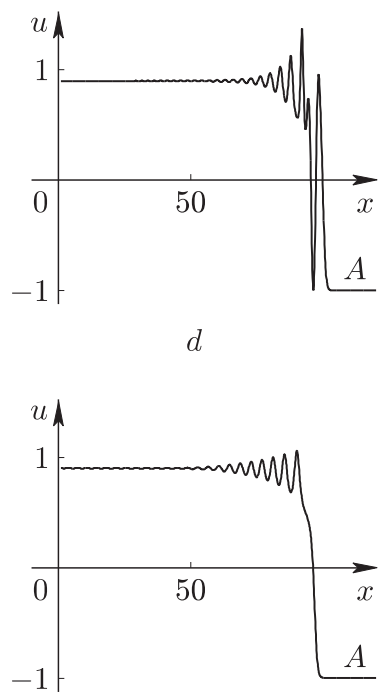

$g$

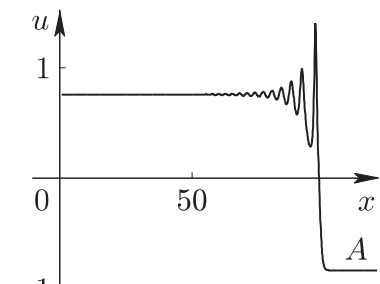

$b$

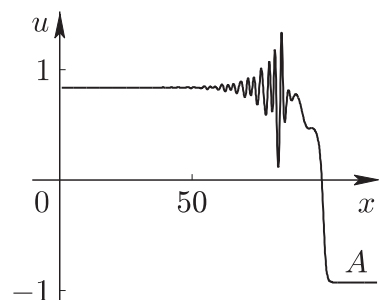

$e$

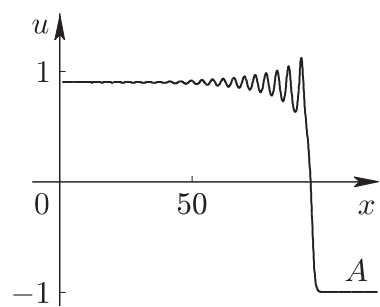

$h$

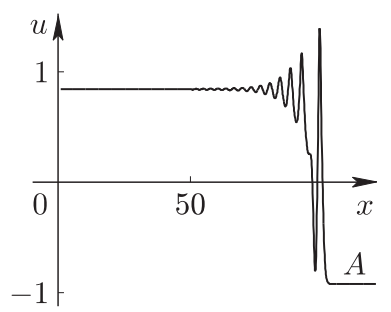

C
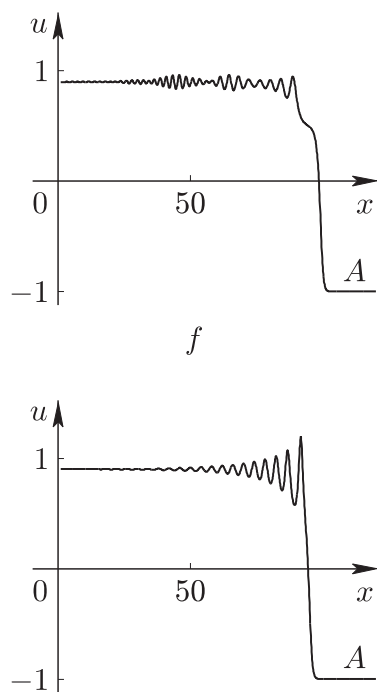

Рис. 4.5. Нестационарное периодическое решение

На рис. $4.5 a-i$ представлено решение $u(x, t)$ в последовательные моменты времени. Состояние $i$ совпадает с состоянием $a$.

Поскольку возмущения не могут уходить на бесконечность в силу диссипативности уравнения (4.2), то равенство (4.4) остается справедливым по отношению к средней скорости разрыва. Заметим, что колебательная нестационарная структура разрыва может существовать при тех же условиях при $x= \pm \infty$, что и стационарная. 
Устойчивость стационарных структур разрывов и, в частности, волн $A \rightarrow C_{i}$ обеспечивает существование таких функций, задающих начальные условия для уравнения (4.2), что при $t \rightarrow \infty$ решения будут иметь асимптотики, описанные в п. 4.1, в том числе асимптотики со стационарными волнами $A \rightarrow C_{i}, i>1$. Такие решения также получались при расчетах.

d) Численно исследованы решения начально-краевых задач, сформулированных в пункте с) при различных значениях параметра $m / \mu^{2}$, различных функциях $\varphi(u)$, описывающих нелинейность, и с граничными условиями $u^{\mathrm{r}}=-1$ и $u^{\mathrm{l}} \in(-1,1)$. Были рассчитаны варианты, представленные в таблице 4.1 .

Представленные в таблице функции имеют простые корни в точках $u= \pm 1$ и двукратный корень при $u=0$. Графики этих функций приведены на рис. 4.6.
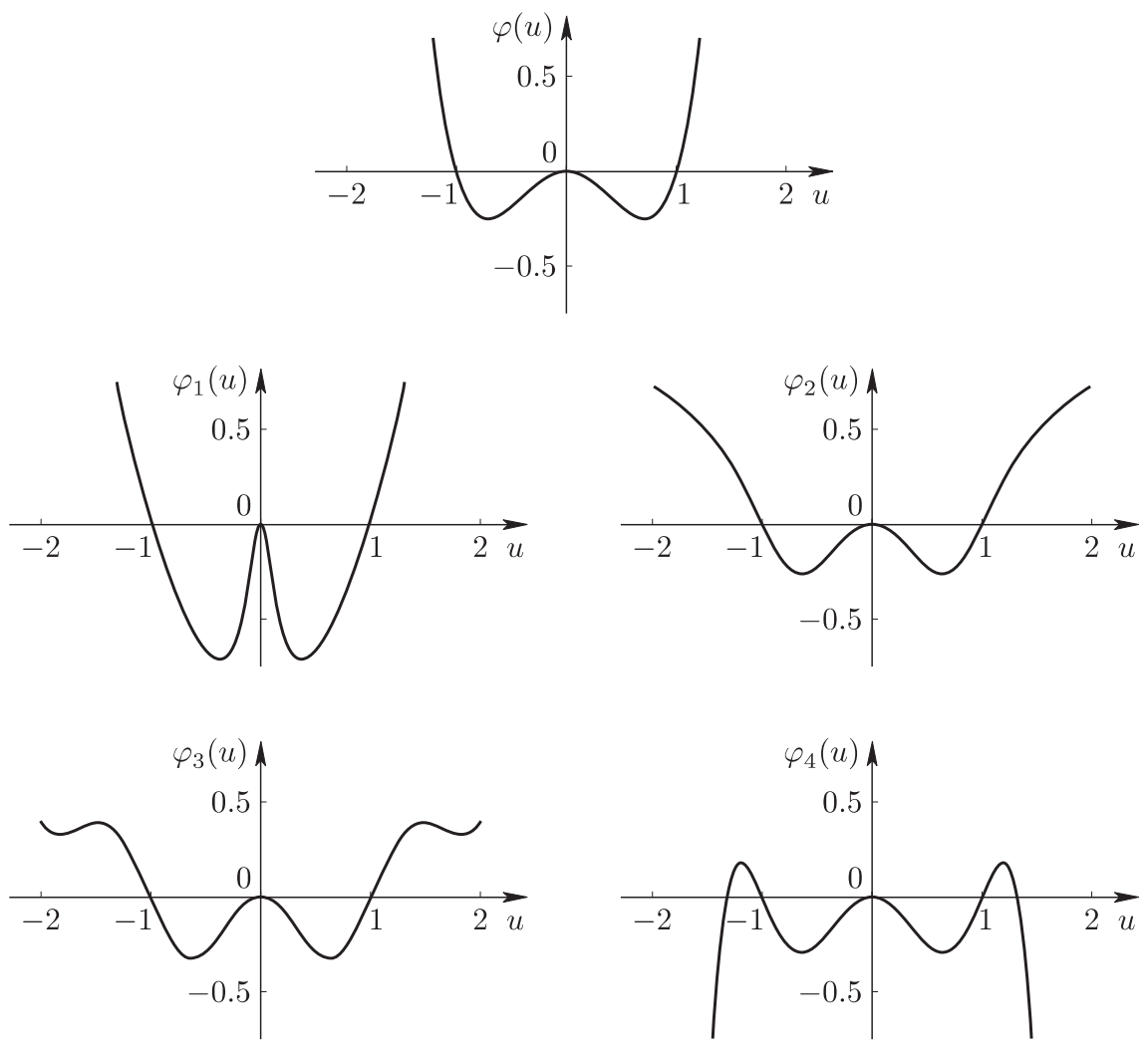

Рис. 4.6. Графики функций $\varphi(u)$ и $\varphi_{i}(u)$

Результаты расчетов аналогов задачи о распаде произвольного разрыва для данных, приведенных в табл. 4.1, качественно совпадают с результатами, описанными в пункте с). В частности, не удалось подобрать такую функцию $\varphi(u)$, для которой не возникали бы разрывы со структурой, периодически зависящей от времени. 
ТАБЛИЦА 4.1

\begin{tabular}{|c|c|c|c|}
\hline$\varphi(u)$ & $m$ & $\mu$ & число точек $C_{i}$ \\
\hline$\varphi(u)=u^{4}-u^{2}$ & 0.42 & 0.05 & 3 \\
$\varphi(u)=u^{4}-u^{2}$ & 0.42 & 0.03 & 7 \\
$\varphi_{1}(u)=\frac{A}{1+u^{2} /(A-1)}+u^{2}-A, A=1.03$ & 2 & 0.05 & 5 \\
$\varphi_{2}(u)=\frac{2 u^{2}\left(u^{2}-1\right)}{2 u^{4}+1.5}$ & 0.55 & 0.03 & 5 \\
$\varphi_{3}(u)=0.2\left(\cos \left(\frac{3 \pi}{2} u\right)+u^{2}-1\right)$ & 0.55 & 0.05 & 5 \\
$\varphi_{4}(u)=\left(\frac{5}{4}\right)^{4} u^{4}-\left(\frac{5}{4}\right)^{2} u^{2}-\left(\frac{5}{16}\right)^{2} u^{6}$ & 0.42 & 0.05 & 5 \\
\hline
\end{tabular}

4.3. Выводы. Таким образом, в результате численных экспериментов было выяснено, что наряду с разрывами в решениях уравнения (4.3), имеющих стационарную структуру, описываемую уравнением (4.2), существуют разрывы, имеющие нестационарную структуру. Разрывы с нестационарной колебательной структурой должны быть включены в множество допустимых разрывов.

Стационарные структуры устойчивы по отношению к одномерным возмущениям, не превышающим некоторого порога. Это утверждение относится также к структуре особых разрывов за исключением особого разрыва с простейшей монотонной структурой, которая не разрушалась при всех рассматриваемых возмущениях.

Если при заданных $u^{\mathrm{r}}$ и $u^{\mathrm{l}}$ может существовать автомодельное решение задачи с разрывом, имеющим нестационарную колебательную структуру, то именно это решение возникает с ростом времени в качестве асимптотики в доопределенной задаче о распаде произвольного разрыва при более или менее произвольных функциях $u_{0}(x)$. Это говорит о том, что автомодельная асимптотика, содержащая разрывы с периодической колебательной структурой, имеет бо́льшую область притяжения в пространстве функций, задающих сглаживание ступеньки в начальных условиях, чем область притяжения автомодельных асимптотик с ударными волнами, имеющими стационарную структуру при тех же значениях параметров задачи.

Таким образом, проведенные расчеты показали, что при заданных значениях параметров $u^{1}, u^{\mathrm{r}}, m / \mu^{2}$ может существовать много автомодельных решений задачи о распаде произвольного разрыва, которые являются асимптотиками соответствующих задач с учетом дисперсии и диссипации. При этом к известным ранее решениям, содержащим разрывы со стационарной структурой, присоединились решения, содержащие разрывы с нестационарной периодической колебательной структурой, обнаруженные численно. 


\section{§ 5. Заключение}

Основная часть результатов работы связана с проблемой неединственности решений нелинейной теории упругости. Было обнаружено (п. 1.3), что решения автомодельных задач, состоящие из волн Римана и априорно эволюционных разрывов с неубывающей энтропией (ударных волн), неединственны. Эта неединственность может иметь место в изотропной среде при сколь угодно малых отклонениях от однородного ненапряженного состояния.

Результаты $\S 2$ связаны с попыткой избавиться от неединственности решений уравнений, описывающих нелинейные квазипоперечные волны в упругой среде, путем введения дополнительной гипотезы о так называемой исчезающей вязкости. При этом решения уравнений теории упругости рассматриваются как предел решений уравнений вязко-упругости Кельвина-Фойхта при вязкости, стремящейся к нулю. Однако ни требования существования структуры разрывов, ни требования устойчивости структуры метастабильных разрывов (т. е. разрывов, которым законы сохранения не препятствуют распасться на систему волн) не привели к каким-либо выводам, способным выделить единственные решения в задачах нелинейной теории упругости. В $\S 2$ на основе изучения неавтомодельных решений с автомодельной асимптотикой, которые строились численно для уравнений вязко-упругости, показано, что осуществление того или иного автомодельного решения как асимптотики при больших временах или при вязкости, стремящейся к нулю, зависит от деталей постановки начальных и граничных условий на отрезках осей $x$ или $t$, длины которых пропорциональны вязкости, и исчезают при вязкости, стремящейся к нулю.

В качестве второго варианта поведения нелинейных квазипоперечных волн в 33 рассмотрен случай, когда в мелкомасшабных процессах, происходящих в упругой среде, помимо вязкости существенную роль играет дисперсия. Требование существования структуры квазипоперечных разрывов выделяет при этом в качестве допустимых разрывов сложно устроенное множество, существенно отличающееся от соответствующего множества при отсутствии дисперсии. При этом помимо ударных волн оказываются допустимыми особые разрывы, скорость распространения которых принимает дискретный набор значений. Число этих дискретных значений тем больше, чем больше относительная роль дисперсии по сравнению с вязкостью в структуре ударных волн. Показано, что число решений автомодельной задачи об упругих волнах в полупространстве, строящихся из волн Римана и допустимых разрывов, может быть велико и оно тем больше, чем больше относительная роль дисперсии в мелкомасштабных процессах.

Численно исследовались неавтомодельные задачи с автомодельной асимптотикой как нестационарные решения уравнений в частных производных, описывающих как крупномасштабные, так и мелкомасштабные процессы, обусловленные вязкостью и дисперсией. Выявлено, что параметры и функции, характеризующие неавтомодельность, определяют асимптотику решения при больших временах. Показано, что, распоряжаясь этими параметрами и функциями, отсутствующими в автомодельной постановке, можно получить в качестве 
асимптотики любое из автомодельных решений. Сделаны выводы о том, какие автомодельные асимптотики реализуются при достаточно простом выборе функций, характеризующих неавтомодельность задачи.

Другая модель, в которой существенную роль играют мелкомасштабные дисперсионные эффекты, описывает распространение продольных волн в вязкоупругих стержнях со сложной нелинейностью. В случае этой модели так же, как и в случае распространения квазипоперечных волн в упругой среде с мелкомасштабной дисперсией, имеют место те же эффекты - сложное строение множества допустимых разрывов, многократная неединственность решений, полученных из непрерывных волн и разрывов, имеющих структуру в виде бегущей волны, а также выявленная при численном построении решений неавтомодельных задач зависимость вида автомодельной асимптотики от параметров и функций, не входящих в автомодельную постановку задачи. Однако имеется отличие. При определенных условиях в асимптотике появляются разрывы, структура которых не представляется бегущей волной, а испытывает внутренние периодические колебания.

На основании проведенных исследований можно сделать вывод, что сложное строение множества допустимых разрывов и множественность автомодельных решений систем уравнений, выражающих законы сохранения, следует ожидать при выполнении двух условий. Необходимо, чтобы по заданному состоянию перед разрывом с одной и той же скоростью мог распространяться более чем один разрыв, удовлетворяющий условиям, следующим из законов сохранения. Кроме того, необходимо, чтобы мелкомасштабная дисперсия существенно превосходила диссипацию в структурах разрывов, что обеспечивает колебания внутри стационарной структуры разрыва.

Во всех рассмотренных случаях, происходит ли отбор допустимых разрывов с помощью введения мелкомасштабной вязкости, или одновременно вязкости и дисперсии, существует область параметров, для которых в рамках гиперболической модели оказывается неединственным решение автомодельной задачи о распаде произвольного разрыва. Выход неавтомодельного решения на ту или иную асимптотику регулируется деталями постановки неавтомодельной задачи, которые отсутствуют в автомодельной постановке.

Это означает, что если, например, требуется в рамках гиперболической модели рассчитать какой-то процесс, в котором по мере его развития возникают произвольные разрывы (например, при столкновении ударных волн), то имеются две возможности. Либо процесс распада этих разрывов следует каждый раз рассчитывать как асимптотику неавтомодельного решения для уравнений, усложненных учетом мелкомасштабных явлений, либо, в ряде случаев, может удасться путем классификации условий возникновения произвольных разрывов и соответствующего численного эксперимента заранее предсказать, каков будет результат распада в изучаемых процессах. Пример последнего подхода продемонстрирован в п. 2.3, где при рассмотрении задачи о взаимодействии ударных волн выяснен тип автомодельного решения, возникающего как асимптотика неавтомодельного решения. 


\section{Список литературы}

[1] Г.Я. Галин, "Об ударных волнах в средах с произвольным уравнением состояния", Докл. АН СССР, 119:6 (1958), 1106-1109; англ. пер.: G. Ya. Galin, "Shock waves in media described by an arbitrary equation of state", Soviet Phys. Dokl., 3 (1958), 244-247.

[2] О.А. Олейник, "О единственности и устойчивости обобщенного решения задачи Коши для квазилинейного уравнения”, УМН, 14:2 (1959), 165-170; англ. пер.: O.A. Olejnik, "Uniqueness and stability of the generalized solution of the Cauchy problem for a quasi-linear equation", Amer. Math. Soc. Transl. (2), 33 (1963), 285-290.

[3] И. М. Гельфанд, "Некоторые задачи теории квазилинейных уравнений", УМН, 14:2 (1959), 87-158; англ. пер.: I. M. Gel'fand, "Some problems in the theory of quasilinear equations", Amer. Math. Soc. Transl. (2), 29 (1963), 295-381.

[4] Г. Я. Галин, "К теории ударных волн”, Докл. АН СCCP, 127:1 (1959), 55-58; англ. пер.: G. Ya. Galin, "A theory of shock waves", Soviet Phys. Dokl., 4 (1960), 757-760.

[5] С. К. Годунов, "О неединственности "размазывания" разрывов в решениях квазилинейных систем", Докл. АН СССР, 136:2 (1961), 272-273; англ. пер.: S. K. Godunov, "On nonunique "blurring" of discontinuities in solutions of quasilinear systems", Soviet Math. Dokl., 2 (1961), 43-44.

[6] В. Ф. Дьяченко, "К задаче Коши для квазилинейных систем", Докл. АН CCCP, 136:1 (1961), 16-17; англ. пер.: V. F. D'yachenko, "Cauchy's problem for quasi-linear systems", Soviet Math. Dokl., 2 (1961), 7-8.

[7] Н. Д. Введенская, "Пример неединственности обобщенного решения квазилинейной системы уравнений”, Докл. АН ССCP, 136:3 (1961), 532-534; англ. пер.: N.D. Vvedenskaya, "An example of nonuniqueness of a generalized solution of a quasi-linear system of equations", Soviet Math. Dokl., 2 (1961), 89-90.

[8] Я.Б. Зельдович, Г. И. Баренблатт, В. Б. Либрович, Г. М. Махвиладзе, Математическая теория горения и взрыва, Наука, M., 1980; англ. пер.: Yа. B. Zel'dovich, G. I. Barenblatt, V.B. Librovich, G. M. Makhviladze, The mathematical theory of combustion and explosions, Consultants Bureau (Plenum), New York, 1985.

[9] А.А. Бармин, А.Г. Куликовский, "Об ударных волнах, ионизующих газ, находящийся в электромагнитном поле”, Докл. АН CCCP, 178:1 (1968), 55-58; англ. пер.: А. A. Barmin; A. G. Kulikovskii, "Shock waves of ionized gases in an electromagnetic field", Soviet Phys. Dokl., 13 (1968), 4-7.

[10] А.Г. Куликовский, "О поверхностях разрыва, разделяющих идеальные среды с различными свойствами. Волны рекомбинации", ПММ, 32:6 (1968), 1125-1131; англ. пер.: A. G. Kulikovskii, "Surfaces of discontinuity separating two perfect media of different properties. Recombination waves in magnetohydrodynamics", J. Appl. Math. Mech., 32:6 (1968), 1145-1152.

[11] Л.И.Седов, В. П. Коробейников, В.В.Марков, "Теория распространения взрывных волн", Тр. МИАН, 175 (1986), 178-216; англ. пер.: L. I. Sedov, V. P. Korobejnikov, V. V. Markov, "The theory of propagation of blast waves", Proc. Steklov Inst. Math., 175 (1988), 187-228.

[12] В. А. Левин, В.В.Марков, Т.А. Журавская, "Нелинейные волновые процессы при инициировании и распространении газовой детонации", Тp. МИАН, 251 (2005), 200-214; англ. пер.: V. A. Levin, V. V. Markov, T. A. Zhuravskaya, "Nonlinear wave processes in the initiation and propagation of gaseous detonation", Proc. Steklov Inst. Math., 251 (2005), 192-205.

[13] Дж. Уизем, Линейные и нелинейные волны, Мир, М., 1977; пер. с англ.: G. B. Whitham, Linear and nonlinear waves, Pure Appl. Math., Wiley, New YorkLondon-Sydney, 1974. 
[14] А. А. Бармин, В. С. Успенский, "Развитие пульсационных режимов в одномерных нестационарных МГД-течениях с выключением электропроводности", Изв. АН СССР, сер. мех. жидк. и газа, 21:4 (1986), 115-122; англ. пер.: А. А. Barmin, V.S. Uspenskii, "Development of pulsation regimes in one-dimensional unsteady MHD flows with switching off of the electrical conductivity", Fluid Dynamics, 21:4 (1986), 604-610.

[15] И.Б. Бахолдин, Бездиссипативные разрывы в механике сплошной среды, Физматлит, М., 2004.

[16] В.Е. Захаров, С. В. Манаков, С.П. Новиков, Л.П. Питаевский, Теория солитонов: Метод обратной задачи, Наука, М., 1980; англ. пер.: S.P. Novikov, S. V. Manakov, L.P. Pitaevskii, V.E. Zakharov, Theory of solitons. The inverse scattering method, Contemp. Soviet Math., Consultants Bureau (Plenum), New York, 1984.

[17] А. Т. Ильичев, Уединенные волны в моделях гидромеханики, Физматлит, М., 2003.

[18] В.И. Карпман, Нелинейнье волны в диспергирующих средах, Наука, М., 1973; англ. пер.: V.I. Karpman, Non-linear waves in dispersive media, International Series of Monographs in Natural Philosophy, 71, Pergamon Press, Oxford-New York-Toronto, ON, 1975.

[19] Л.И. Седов, Механика сплошной средъ, т. 2, 2, Наука, М., 1994; англ. пер.: L. I. Sedov, Mechanics of continuous media, II, World Scientific, River Edge, NJ, 1997.

[20] Д.Р. Бленд, Нелинейная динамическая теория упругости, Мир, М., 1972; пер. с англ.: D. R. Bland, Nonlinear dynamic elasticity, Blaisdell, Waltham, MA-Toronto, ON-London, 1969.

[21] А. Г. Куликовский, Е. И. Свешникова, Нелинейные волны в упругих средах, Моск. Лицей, М., 1998; пер. с англ.: А. G. Kulikovskii, E. I. Sveshnikova, Nonlinear waves in elastic media, CRC Press, Boca Raton, FL, 1995.

[22] А. Г. Куликовский, Н. В. Погорелов, А. Ю. Семенов, Математические вопросы численного решения гиперболических систем уравнений, Физматлит, М., 2001.

[23] В.В.Лохин, Л.И.Седов, "Нелинейные тензорные функции от нескольких тензорных аргументов", ПМM, 27:3 (1963), 393-417; англ. пер.: V. V. Lokhin, L. I. Sedov, "Nonlinear tensor functions of several tensor arguments", J. Appl. Math. Mech., 27:3 (1963), 597-629.

[24] А. Г. Куликовский, "Об уравнениях, описывающих распространение нелинейных квазипоперечных волн в слабоанизотропном упругом теле”, ПМM, 50:4 (1986), 597-604; англ. пер.: A. G. Kulikovskii, "Equations describing the propagation of nonlinear quasitransverse waves in a weakly non-isotropic elastic body", J. Appl. Math. Mech., 50:4 (1986), 455-461.

[25] Е.И. Свешникова, "Простые волны в нелинейно упругой среде", ПММ, 46:4 (1982), 642-646; англ. пер.: E. I. Sveshnikova, "Simple waves in nonlinearly elastic media", J. Appl. Math. Mech., 46:4 (1982), 509-512.

[26] А. Г. Куликовский, Е. И. Свешникова, "Исследование ударной адиабаты квазипоперечных ударных волн в предварительно напряженной упругой среде", ПММ, 46:5 (1982), 831-840; англ. пер.: A. G. Kulikovskii, E. I. Sveshnikova, "Investigation of the shock adiabat of quasitransverse shock waves in a prestressed elastic medium", J. Appl. Math. Mech., 46:5 (1982), 667-673.

[27] Л. Д. Ландау, Е. М. Лифшиц, Теоретическая физика. VI: Гидродинамика, Наука, M., 1986; нем. пер.: L. D. Landau, E. M. Lifschitz, Lehrbuch der theoretischen Physik. VI: Hydrodynamik, Akademie-Verlag, Berlin, 1991.

[28] P. D. Lax, "Hyperbolic systems of conservation laws. II", Comm. Pure Appl. Math., 10:4 (1957), 537-566. 
[29] А.Г. Куликовский, Е.И. Свешникова, "Автомодельная задача о действии внезапной нагрузки на границу упругого полупространства", ПММ, 49:2 (1985), 284-291; англ. пер.: A. G. Kulikovskij, E. I. Sveshnikova, "A selfsimilar problem on the action of a sudden load on the boundary of an elastic half-space", J. Appl. Math. Mech., 49:2 (1985), 214-220.

[30] А. Г. Куликовский, Е. И. Свешникова, "О распаде произвольного начального разрыва в упругой среде”, ПММ, 52:6 (1988), 1007-1012; англ. пер.: A. G. Kulikovskij, E. I. Sveshnikova, "The decay of an arbitrary initial discontinuity in an elastic medium", J. Appl. Math. Mech., 52:6 (1988), 786-790.

[31] А. Г. Куликовский, Е. И. Свешникова, "Признак несуществования и неединственности решений автомодельных задач механики сплошной среды”, ПММ, 65:6 (2001), 971-982; англ. пер.: А. G. Kulikovskij, E. I. Sveshnikova, "A condition for the nonexistence and nonuniqueness of solutions of self-similar problems of continuum mechanics", J. Appl. Math. Mech., 65:6 (2001), 941-950.

[32] А.Г. Куликовский, "Особенности поведения нелинейных квазипоперечных волн в упругой среде при малой анизотропии", Тр. МИАН, 186 (1989), 132-139; англ. пер.: A. G. Kulikovskij, "Peculiarities of the behavior of nonlinear quasitransversal waves in an elastic medium with a small anisotropy", Proc. Steklov Inst. Math., 186 (1991), 153-160.

[33] А.Г. Куликовский, Е.И. Свешникова, "О структуре квазипоперечных упругих ударных волн", ПММ, 51:6 (1987), 926-932; англ. пер.: A. G. Kulikovskij, E. I. Sveshnikova, "On the structure of quasitransverse elastic shock waves", J. Appl. Math. Mech., 51:6 (1987), 711-716.

[34] А. П. Чугайнова, "Исследование структуры квазипоперечных ударных волн для определенного класса упругих сред”, Проблемы механики, экологии, технологии, Наука, М., 1991.

[35] А.П. Чугайнова, "О формировании автомодельного решения в задаче о нелинейных волнах в упругом полупространстве", ПМM, 52:4 (1988), 692-697; англ. пер.: A.P. Chugainova, "The formation of a selfsimilar solution for the problem of non-linear waves in an elastic half-space", J. Appl. Math. Mech., 52:4 (1988), 541-545.

[36] А.П. Чугайнова, “О выходе нелинейных волн на автомодельный режим в задаче о действии внезапного изменения нагрузки на границу упругого полупространства", Изв. АН СССР, сер. мех. тверд. тела, 25:3 (1990), 187-189.

[37] А. П. Чугайнова, "О взаимодействии нелинейных волн в слабоанизотропной упругой среде", ПММ, 57:2 (1993), 149-156; англ. пер.: A.P. Chugainova, "The interaction of non-linear waves in a slightly anisotropic viscoelastic medium", J. Appl. Math. Mech., 57:2 (1993), 375-381.

[38] А.П. Чугайнова, "О перестройке нелинейной упругой волны в среде с малой анизотропией", Изв. РАН, сер. мех. тверд. тела, 28:5 (1993), 75-81.

[39] Б. Л. Рождественский, Н.Н. Яненко, Системы квазилинейных уравнений и их приложения к газовой динамике, Наука, M., 1978; англ. пер.: B. L. Roždestvenskii, N.N. Janenko, Systems of quasilinear equations and their applications to gas dynamics, Transl. Math. Monogr., 55, Amer. Math. Soc., Providence, RI, 1983.

[40] А.Г. Куликовский, А.П. Чугайнова, "Об условиях распада нелинейной волны в вязко-упругой среде", ЖКМ и МФ, 38:2 (1998), 315-323; англ. пер.: A. G. Kulikovskij, A.P. Chugainova, "Disintegration conditions for a nonlinear wave in a viscoelastic medium", Comput. Math. Math. Phys., 38:2 (1998), 305-312.

[41] А.Г. Куликовский, А.П. Чугайнова, "Об устойчивости к двумерным возмущениям метастабильной ударной волны в вязко-упругой среде", ПMM, 66:1 (2002), 109-117; англ. пер.: А. G. Kulikovskij, A.P. Chugainova, "The stability of a metastable shock wave in a viscoelastic medium under two-dimensional perturbations", J. Appl. Math. Mech., 66:1 (2002), 107-114. 
[42] А. Г. Куликовский, А. П. Чугайнова, "Об устойчивости квазипоперечных ударных волн в анизотропных упругих средах", ПММ, 64:6 (2000), 1020-1026; англ. пер.: A. G. Kulikovskij, A.P. Chugainova, "The stability of quasi-transverse shock waves in anisotropic elastic media", J. Appl. Math. Mech., 64:6 (2000), 981-986.

[43] Б. Б. Кадомцев, В. И. Петвиашвили, "Об устойчивости уединенных волн в слабо диспергирующих средах", Докл. АН СССР, 192:4 (1970), 753-756; англ. пер.: B. B. Kadomtsev, V.I. Petviashvili, "The stability of quasi-transverse shock waves in anisotropic elastic media", Soviet Phys. Dokl., 15 (1970), 539-541.

[44] Е.А. Заболотская, Р. В. Хохлов, "Квазиплоские волны в нелинейной акустике ограниченных пучков", Ажустический журнал, 15:1 (1969), 40-47.

[45] А.Г. Куликовский, "О возможном влиянии колебаний в структуре разрыва на множество допустимых разрывов", Докл. АН СССР, 275:6 (1984), 1349-1352; англ. пер.: A. G. Kulikovskii, "The possible effect of oscillations in a discontinuity structure on the set of admissible discontinuities", Soviet Phys. Dokl., 29:4 (1984), 283-285.

[46] А.Г. Куликовский, Н. И. Гвоздовская, "О влиянии дисперсии на множество допустимых разрывов в механике сплошной среды", Тр. МИАН, 223 (1998), 63-73; англ. пер.: A. G. Kulikovskii, N. I. Gvozdovskaya, "The effect of dispersion on the set of admissible discontinuities in continuum mechanics", Proc. Steklov Inst. Math., 223 (1998), 55-65.

[47] А.П. Чугайнова, "Асимптотическое поведение нелинейных волн в упругих средах с дисперсией и диссипацией”, ТМФ, 147:2 (2006), 240-256; англ. пер.: A.P. Chugainova, "Asymptotic behavior of nonlinear waves in elastic media with dispersion and dissipation", Theoret. Math. Phys., 147:2 (2006), 646-659.

[48] А.П. Чугайнова, “Автомодельные асимптотики волновых задач и структуры неклассических разрывов в нелинейно-упругих средах с дисперсией и диссипацией", ПМM, 71:5 (2007), 775-787; англ. пер.: А.P. Chugainova, "Self-similar asymptotics of wave problems and the structures of non-classical discontinuities in non-linearly elastic media with dispersion and dissipation", J. Appl. Math. Mech., 71:5 (2007), 701-711.

[49] Н. С. Бахвалов, М. Э. Эглит, “Эффективные уравнения с дисперсией для распространения волн в периодических средах", Докл. РАН, 370:1 (2000), 7-10; англ. пер.: N.S. Bakhvalov, M. ̇̇. Èglit, "Effective equations with dispersion for waves propagating in periodic media", Dokl. Math., 61:1 (2000), 1-4.

[50] А. Г. Куликовский, А. П. Чугайнова, "Моделирование влияния мелкомасштабных дисперсионных процессов в сплошной среде на формирование крупномасштабных явлений", ЖВМ и $М \Phi, 44: 6$ (2004), 1119-1126; англ. пер.: A. G. Kulikovskij, A.P. Chugajnova, "The simulation of influence of small-scale dispersive processes in a solid medium on the formation of large-scale phenomena", Comput. Math. Math. Phys., 44:6 (2004), 1062-1068.

[51] Д. В. Релей, Теория звука, Гостехиздат, M., 1955; пер. с англ.: J. W. S. Rayleigh, The theory of sound, Dover, New York, 1945.

А. Г. Куликовский (А. G. Kulikovskii)

Математический институт им. В. А. Стеклова РАН

E-mail: kulik@mi.ras.ru
Поступила в редакцию 05.02.2008

\section{А. П. Чугайнова (А. P. Chugainova)}

Математический институт им. В. А. Стеклова РАН

E-mail: A.P.Chugainova@mi.ras.ru 